\title{
Haptic Training Simulator for Pedicle Screw Insertion in Scoliosis Surgery
}

\author{
by \\ SeyedehMaryam Moafimadani
}

\author{
A thesis \\ presented to the University of Waterloo \\ in fulfillment of the \\ thesis requirement for the degree of \\ Master of Applied Science \\ in \\ Electrical and Computer Engineering
}

Waterloo, Ontario, Canada, 2015

(c) SeyedehMaryam Moafimadani 2015 


\section{Author's Declaration}

I hereby declare that I am the sole author of this thesis. This is a true copy of the thesis, including any required final revisions, as accepted by my examiners.

I understand that my thesis may be made electronically available to the public. 


\begin{abstract}
This thesis develops a haptic training simulator that imitates the sensations experienced by a surgeon in pedicle screw insertions in a scoliosis surgery.

Pedicle screw insertion is a common treatment for fixing spinal deformities in idiopathic scoliosis. Surgeons using the free hand technique are guided primarily by haptic feedback. A vital step in this free hand technique is the use of a probe to make a channel through the vertebrae pedicle. This is a sensitive process which carries risk of serious mechanical, neurological and vascular complications. Surgeons are currently trained using cadavers or live patients. Cadavers often have vertebrae that are softer than the real surgeons would typically encounter, while training on live patients carries the obvious issue of increased risk of complications to the patient. In this thesis, a haptic virtual reality simulator is designed and studied as a training tool for surgeons in this procedure.

Creating a pathway through the pedicle by the free-hand technique is composed of two main degrees of freedom: rotation and linear progression. The rotary stage of the device which was developed by a previous student, is enhanced in this research by adding hardware, improving the haptic model and proposing techniques to couple the rotary and linear degree of freedom. Haptic model parameters for a spine surgery with normal bone density are then clinically tuned within a user study. Over ten surgeons of varying experience levels used the simulator and were able to change various parameters in order to tune the simulator to what felt most realistic. The surgeons also evaluated the simulator for its feasibility and usefulness. Four research questions were investigated. First, can a reference set of values be found that replicate the surgeon's interpretation of the surgical scenario? Second, how are the rotary stage parameters influenced in the presence of linear effects? Third, do the results differ across different expertise levels? Finally, can the simulator serve
\end{abstract}


as a useful tool in the education of surgical trainees for teaching channel creation in pedicle screw insertion? Statistical analysis are carried out to examine the research questions. The results indicates the feasibility of the simulator for surgical education. 


\section{Acknowledgements}

I would like to express my sincere gratitude to my supervisor, Dr. David Wang, for giving me the opportunity to pursue my Master's degree and providing the support to work on this interesting project. I thank him for his constant support and encouragement which has been with me in all the steps of my research. I owe him gratitude for generously mentoring me and guiding me to opportunities which were not limited to my academic life.

I would like to thank Dr. Karl Zabjek at University of Toronto for his endless support throughout the project. Sincere thanks as well for his efforts in recruiting participants throughout the clinical testing.

I thank Dr. Reinhard Zeller for his invaluable clinical knowledge and expertise and being open to provide constructive feedback throughout the project. Sincere thanks to Dr. Massicotte for providing me the opportunity to attend the pedicle screw surgery workshops

where I gained precious insights through surgical observation and hands on participation with cadavers.

Also, to people at the University of Waterloo, especially members of my research team, in particular Kevin Kraul for his vital advice through the development of the experimental apparatus. Special thanks to Regina Leung for showing limitless enthusiasm for introducing me to this project and providing me with her valuable feedback whenever requested. I thank Yi Wang for her help throughout data collection that made the last part of the project so enjoyable and much more productive.

My deepest gratitude goes to my parents and brothers for their encouragement and support throughout this journey. 


\section{Table of Contents}

List of Tables $\quad$ x

List of Figures $\quad$ xi

1 Introduction $\quad 1$

1.1 Idiopathic Scoliosis Epidemiology and Classification System . . . . . . . 1

1.2 Steps in Pedicle Screw Insertion . . . . . . . . . . . . . . 6

1.3 Surgery Complications and Reducing Surgical Risks . . . . . . . . . . 10

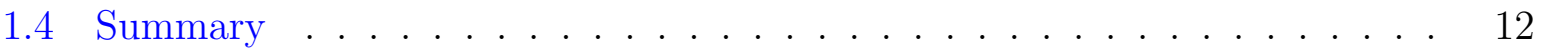

2 Background $\quad 13$

2.1 Medical Training Simulators . . . . . . . . . . . . . . . . 13

2.2 Haptics .............................. 16

2.3 Haptic Training Simulators for Medical Applications . . . . . . . . . . . . 19

2.4 Haptic Training Simulators for Pedicle Screw Insertion . . . . . . . . . . 19

2.5 A Summary on the Progress of the Project by Previous Researcher . . . . 22 
2.6 Summary . . . . . . . . . . . . . . . . . . . . . 23

3 Biomechanical Characteristics of the Surgical Procedure 24

3.1 Scenarios Considered for Simulation . . . . . . . . . . . . . . . . . 27

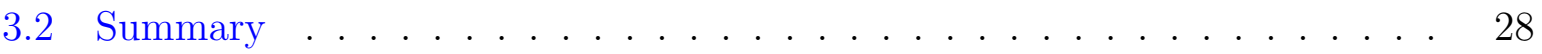

4 Methodology $\quad 29$

4.1 Research Methodology . . . . . . . . . . . . . . . . . . 29

4.2 Research and Design Objectives . . . . . . . . . . . . . . . 32

4.3 Control Structure . . . . . . . . . . . . . . . . . 33

4.3.1 Summary of the Rotary Stage Haptic Model . . . . . . . . . . . . . 34

4.4 Potential Control Structures for Simulation of the Linear Haptic Effects . . 36

4.4.1 Closed Loop PID Control . . . . . . . . . . . . . . . . . . 38

4.4.1.1 Linear Progression Simulation . . . . . . . . . . . 39

4.4.1.2 Breach Simulation . . . . . . . . . . . . . . 40

4.4 .2 Open Loop Control . . . . . . . . . . . . . . . . . 41

4.4.2.1 Linear Progression Simulation . . . . . . . . . . . 41

4.4.2.2 Breach Simulation . . . . . . . . . . . . . . 42

4.5 Coupling Rotary Haptic Effects with Linear Haptic Effects . . . . . . . . . 43

4.6 Summary . . . . . . . . . . . . . . . . . . . . . 44 
5.1 The Rotary Stage Platform _ . . . . . . . . . . . . . . . . 45

5.2 Design Components of the Completed Haptic Device . . . . . . . . . . 47

5.2 .1 Hardware Components . . . . . . . . . . . . . . . . . . . . 47

$5.2 .2 \quad$ Hardware/Software Interface $\ldots \ldots \ldots \ldots \ldots$

5.2 .3 Software and Real-time Component . . . . . . . . . . . . . 52

5.2.4 Design and Implementation of the Proposed Control Techniques . . 53

5.2.4.1 Closed loop PID Control Technique: Design and Compu-

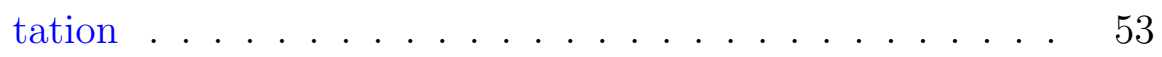

5.2.5 Open Loop Control: Design and Computation . . . . . . . . . 66

5.3 Summary of Haptic Model Parameters . . . . . . . . . . . . . . . . . 68

5.4 Summary . . . . . . . . . . . . . . . . . . . . . . 69

$\begin{array}{lll}6 & \text { Clinical Testing } & 70\end{array}$

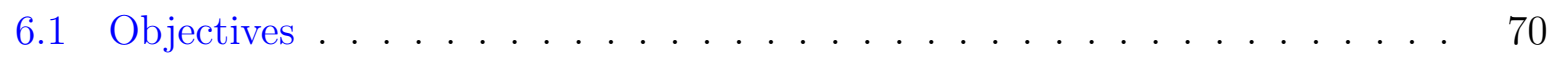

6.2 Ethics Statement . . . . . . . . . . . . . . . . . . . . . . 71

6.3 Research Questions . . . . . . . . . . . . . . . . . . . . . . 71

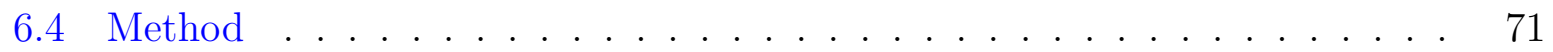

6.5 Participants . . . . . . . . . . . . . . . . . . . 73

6.6 Protocol Outline . . . . . . . . . . . . . . . . . 73

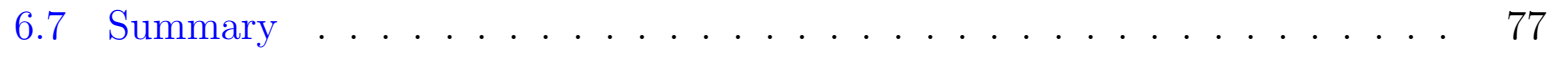


$\begin{array}{lll}7 & \text { Discussion } & 78\end{array}$

7.1 Pilot Studies Prior to Official Testing . . . . . . . . . . . . . 78

7.2 Discussion on the Results from Official Testing . . . . . . . . . . . . . 80

7.2.1 Discussion of Research Question No. 1 . . . . . . . . . . . . 81

7.2.2 Discussion of Research Question No. 2 . . . . . . . . . . . 84

7.2.3 Discussion of Research Question No. 3 . . . . . . . . . . . 85

7.2.4 Discussion of Research Question No. 4 . . . . . . . . . . . . 90

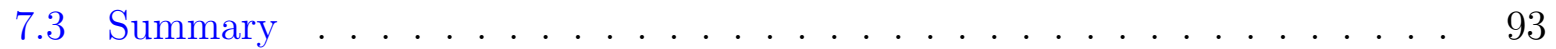

8 Conclusion and Future Works $\quad 94$

8.1 General Conclusion . . . . . . . . . . . . . . . . . . . 94

8.2 Future Work . . . . . . . . . . . . . . . . . . 96

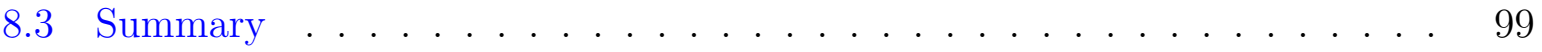

$\begin{array}{lr}\text { APPENDICES } & 100\end{array}$

$\begin{array}{ll}\text { A System Components } & 101\end{array}$

A.1 Graphical User Interface . . . . . . . . . . . . . . . . 103

$\begin{array}{ll}\text { B Ethics Material } & 106\end{array}$

$\begin{array}{ll}\text { References } & 111\end{array}$ 


\section{List of Tables}

2.1 Pros and cons of conventional training approaches . . . . . . . . . . 15

5.1 The mean squared error between the measured signal and output of adjusted models . . . . . . . . . . . . . . . . . . . . . . . . . 59

5.2 Experimental result for measuring static friction . . . . . . . . . . . 61

6.1 Terminology used in testing . . . . . . . . . . . . . . . . 75

7.1 Summary statistics . . . . . . . . . . . . . . . . . . . . . 83

7.2 Comparison of current study's rotary stage parameters with previous study 84

7.3 Levene's statistical test result . . . . . . . . . . . . . . . 86

7.4 Summary statistic based on expertise level . . . . . . . . . . . . . . . 87

7.5 ANOVA test result $\ldots \ldots \ldots \ldots \ldots \ldots$

7.6 Welch test result . . . . . . . . . . . . . . . . . . . . 88

7.7 Comparing satisfaction rating of simulator realism and teaching tool potential to an agreement benchmark $(3.5 / 5.0) \ldots \ldots \ldots \ldots . \ldots . \ldots 92$ 


\section{List of Figures}

1.1 Normal spine column from lateral and posterior view . . . . . . . . . . 3

1.2 Some common curve patterns in scoliosis . . . . . . . . . . . . . . . 4

1.3 Back and side view of a female adolescent patient ( $a, b)$ before surgery (c, d) after bilateral pedicle screw instrumentation . . . . . . . . . 5

1.4 Cross-section of vertebrae . . . . . . . . . . . . . . . 6

1.5 Steps in pedicle screw insertion . . . . . . . . . . . . . . . 7

1.6 Posterior of a cadaver's vertebrae which is exposed for research purpose . . 9

1.7 Computed tomography (CT) scan of screws inside pedicle. . . . . . . . . 11

3.1 Possible types of breakthrough . . . . . . . . . . . . . . . 26

4.1 Research model . . . . . . . . . . . . . . . . . . . 30

4.2 General control diagram of the haptic training simulator . . . . . . . . . 33

4.3 Proportional component of the rotary stage control signal . . . . . . . . . 35

4.4 Block diagram of the PID control scheme . . . . . . . . . . . . . . 38

4.5 Inside the nonlinear block . . . . . . . . . . . . . . . . . 39 
4.6 Trajectory planner used for the simulation of breach effects in closed loop PID control . . . . . . . . . . . . . . . . . . . . . 40

4.7 Block diagram of the open loop control scheme . . . . . . . . . . . . . . 41

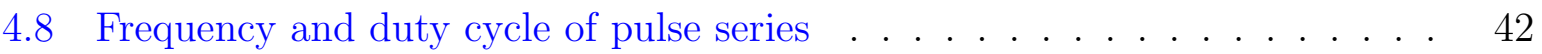

4.9 Trajectory planner used for the simulation of breach effects in open loop control . . . . . . . . . . . . . . . . . . . . . .

5.1 First stage of the haptic training simulator . . . . . . . . . . . . . 46

5.2 Linear stage showing position sensor, linear actuator, and supporting aluminum posts . . . . . . . . . . . . . . . . . . . 48

5.3 Overview of the haptic simulator $\ldots \ldots \ldots \ldots \ldots$

5.4 System components . . . . . . . . . . . . . . . . . . . . . . . . . 52

5.5 Frequency response of the identified model vs. the actual output response 57

5.6 Velocity response estimation of identified model vs actual measurements . . 58

5.7 Making adjustment to the parameters a and $\tau$. . . . . . . . 60

5.8 Comparing open loop bode plots; first one is without the controller, second one is with the initial controller and last one is with modified controller (adjusted for step size of 10mm) . . . . . . . . . . . . . . . .

5.9 Output response of the system with the controller tuned for $5 \mathrm{~mm}$ step size on a set of steps as a reference . . . . . . . . . . . . . . . . . .

5.10 Output response of the system with the controller tuned for $40 \mathrm{~mm}$ step size on a set of steps as a reference . . . . . . . . . . . . . 
5.11 Displacement of the actuator with maximum input voltage and six different pulse duration including $0.3,0.25,0.2,0.15,0.1$ and $0.05 \ldots \ldots$. . . . 67

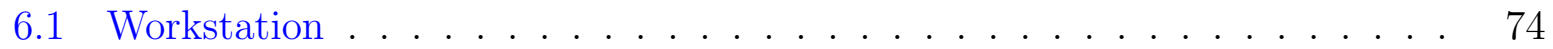

7.1 Distribution of parameter values obtained from clinical testing with experienced surgeons. Outliers are excluded from the data set. Confidence interval for each parameter is indicated by green horizontal lines . . . . . . . . . .

7.2 Comparison of variance among surgeons with different expertise level with respect to mean, outliers are removed . . . . . . . . . . . . . . .

7.3 Summary of questionnaire results for 11 surgeon participants . . . . . . . . 91

8.1 Force and torque profile . . . . . . . . . . . . . . . . 98

A.1 Graphical user interface for the experiment on breach and linear progression

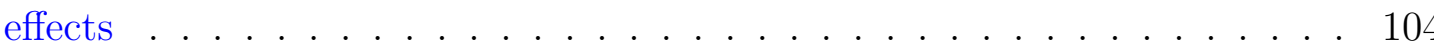

A.2 Graphical user interface for the experiment on breach and linear progression

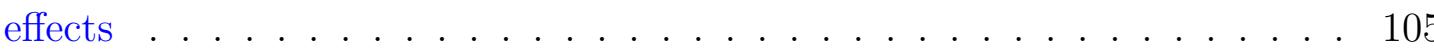




\section{Chapter 1}

\section{Introduction}

Idiopathic scoliosis surgery is a very common treatment for patients with spinal curvature. It is risky surgery and it is often hard to train new surgeons. In order to reduce the complications of surgery, we aim to develop a training simulator to improve the surgeons' technical skills prior to operating on live patients.

\subsection{Idiopathic Scoliosis Epidemiology and Classifica- tion System}

Idiopathic scoliosis is defined as a lateral curvature of the spine greater than 10 degrees with unknown cause. The prevalence of the disorder is equal among males and females, but the severity of curvature is generally more serious in females. Curves greater than 30 degrees are 10 times more common in females than males [1].

In most cases of scoliosis, etiology remains unclear and the disorder is thought to often be due to genetic factors. The reasons for treatment include improving physical 
appearance, reducing back pain, promoting physical comfort, and preventing excessive curve. If untreated at a young age, the severity of deformity increases and may result in complications such as breathing difficulty, rib cage rotation, heart problems, loss of shoulder balance [2, 3].

Scoliosis is classified into three groups based on the age at initial diagnosis: adolescent idiopathic scoliosis (AIS), juvenile scoliosis and infantile scoliosis. The age at diagnosis for these categories are over 10 years of age, between 9 and 4 years of age, and 3 years of age or younger, respectively [2]. Many schools regularly screen students in an aim to diagnose scoliosis at an early age. The lack of evidence supporting screening and the cost have led some to discourage mass screening, while the great benefits of early intervention in children with scoliosis is used by others to justify screening [4].

Curves of the spine are classified depending on their shape, location, pattern, and cause. Deformity could be with one curve or two curves and makes the spine C-shaped or S-shaped. Location is identified by finding the location of the apex of the curve. The apex can be in the thoracic, lumbar and thoracolumbar regions of the spine. Scoliosis and the degree of curvature are usually evaluated by spinal radiography. Figure 1.1 shows a normal spine with these areas identified. Patterns are grouped according to the convexity to the right or left of the curve points. Some curve patterns are shown in Figure 1.2. In AIS, the most common curve pattern consists of a thoracic curve (usually to the right) with or without a thoracolumbar or lumbar curve (usually to the left) [4]. 


\section{Normal Spine}
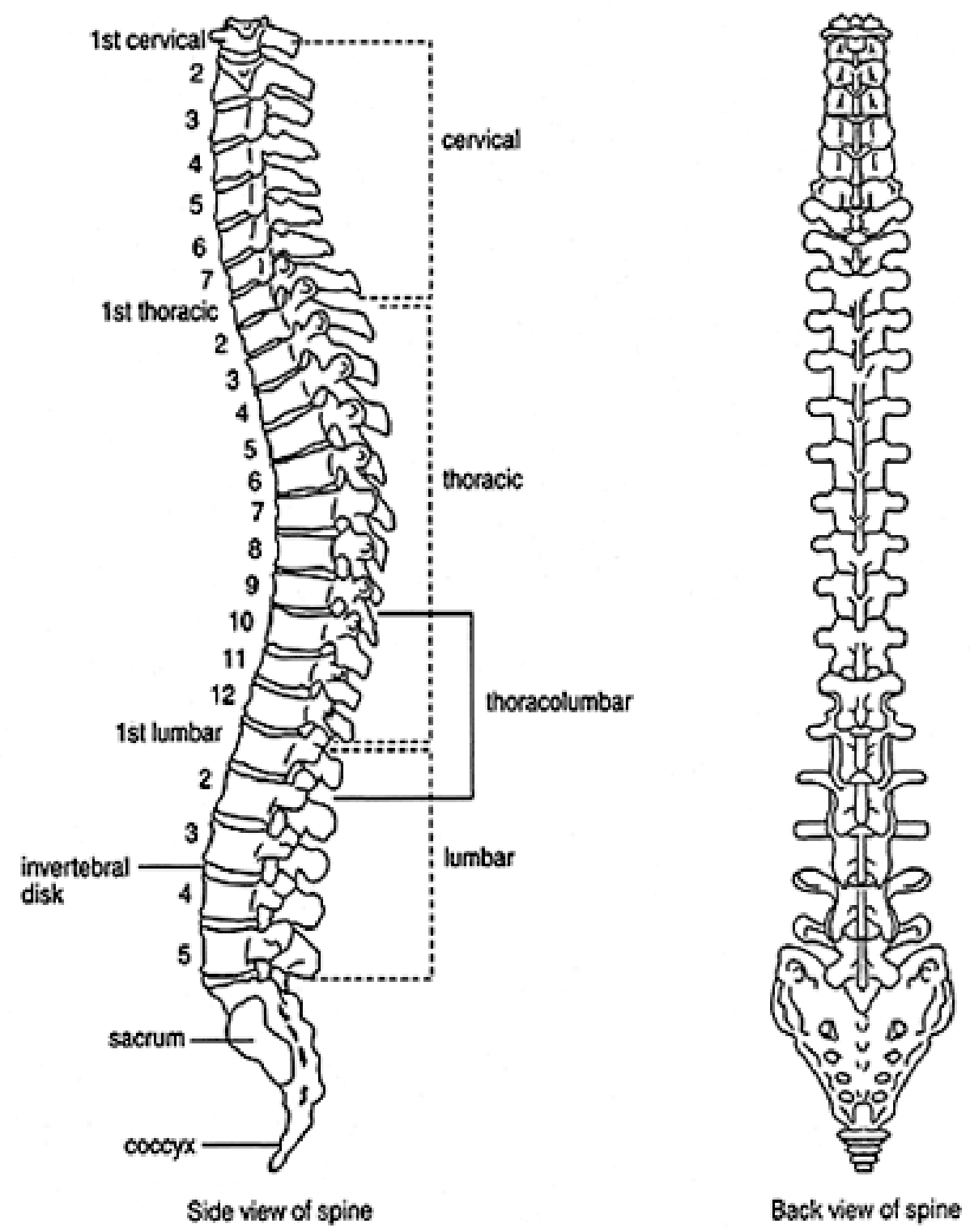

Figure 1.1: Normal spine column from lateral and posterior view [5] 


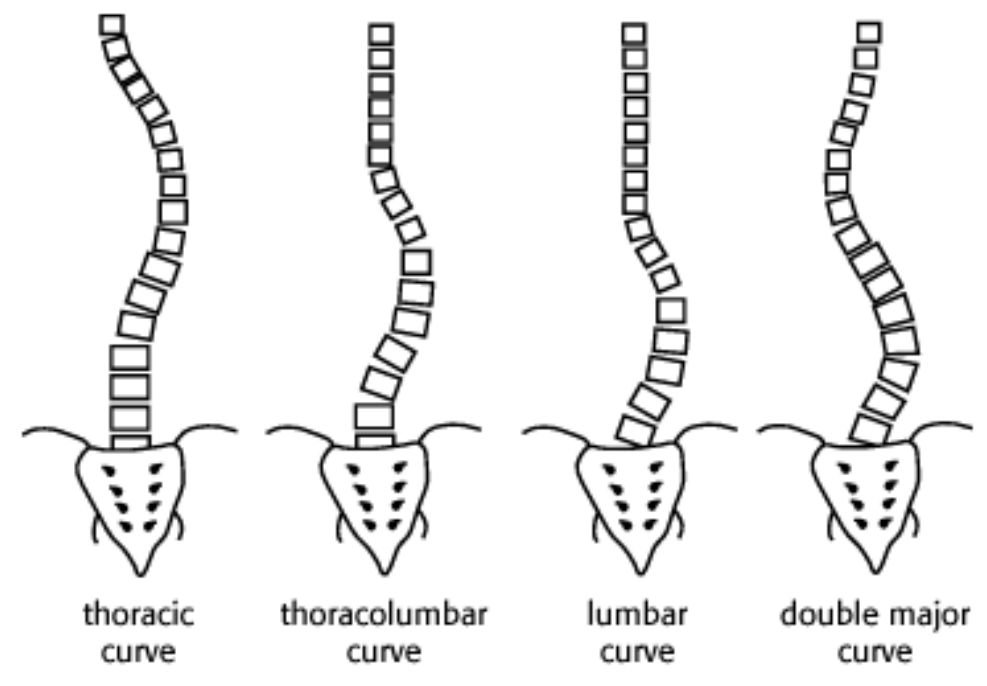

Figure 1.2: Some common curve patterns in scoliosis [6]

The treatment method required depends on the type and degree of the curve. There are three main treatment options, including observation, back bracing, and scoliosis surgery.

\section{Observation}

For a mild curve, the patient is observed by a physician regularly to monitor the degree of curvature. School screening programs fall into this category.

\section{Back bracing}

This treatment method uses braces for preventing or slowing curvature progression and may decrease the need for later surgery. This method can be effective for skeletally immature patients $[2,3]$.

\section{Scoliosis surgery}

This treatment is mainly used for correcting curves of greater than 50 degrees, or 
for patients with spinal curvature of less than 50 degrees who are still growing [2]. Figure 1.3 shows radiographs of a patient's vertebrae prior and after scoliosis surgery.
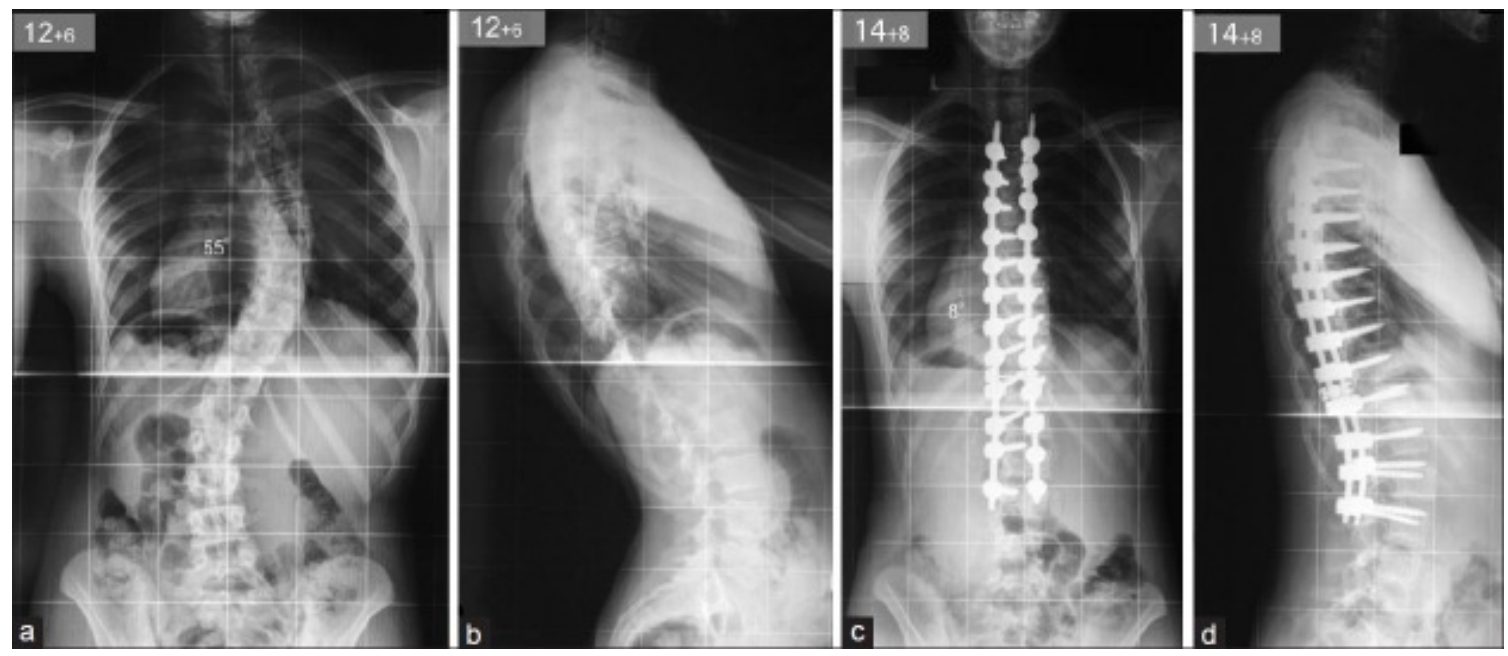

Figure 1.3: Back and side view of a female adolescent patient (a, b) before surgery (c, d) after bilateral pedicle screw instrumentation [7]

The hook-and-rod system and pedicle screw instrumentation are the most common surgical treatments. Pedicle is a tubular bone that connects the vertebral body to the lamina. As shown in Figure 1.4 each vertebra has two pedicles.

The hook-and-rod system is an older technique and is usually used for small pedicle sizes in young patients [4]. In pedicle screw instrumentation, screws are placed through the pedicle and inside the vertebral body, and then connected by a short rod which straightens the spine. This construct proves to be biomechanically effective for segmental fixation [8]. Pedicle screw instrumentation has many advantages including superior three-column mechanical fixation and multi-planar corrections [3]. Pedicle screws enable gripping spinal segments. 


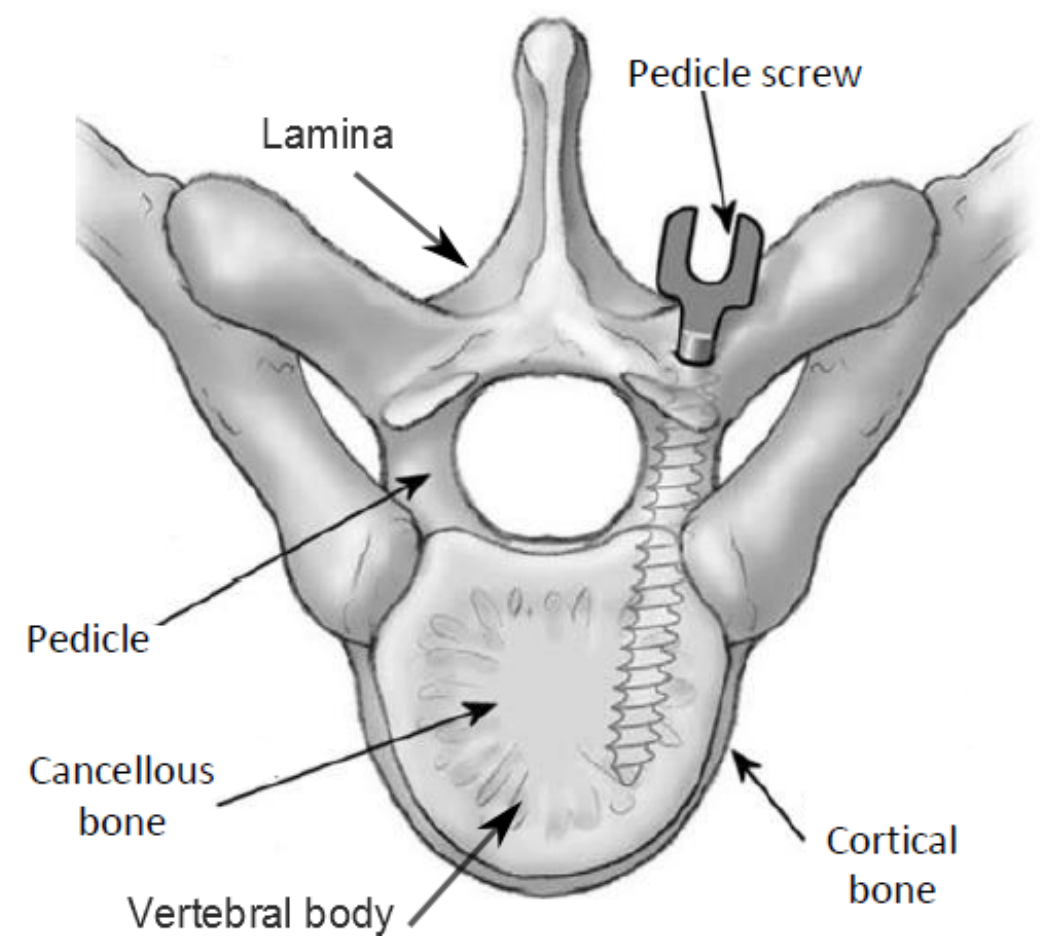

Figure 1.4: Cross-section of vertebrae [9]

\subsection{Steps in Pedicle Screw Insertion}

This type of surgery is often conducted using the free-hand anatomic technique and relies on visual as well as haptic feedback [10, 7]. The following are the steps included in typical pedicle screw insertion using the free-hand technique [11, 12, 13] which are also illustrated in Figure 1.5: 

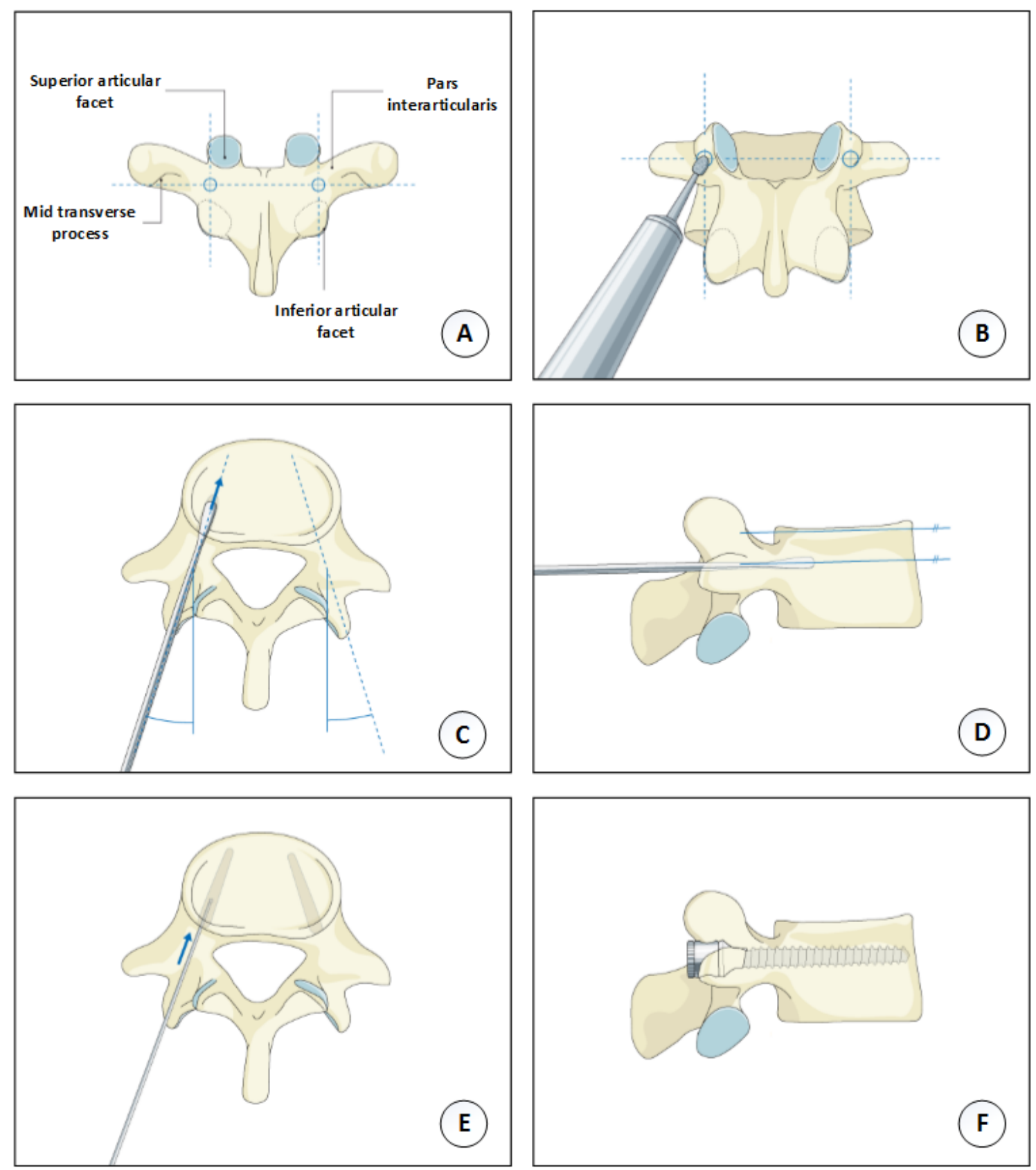

Figure 1.5: Steps in pedicle screw insertion. (A) pedicle screw entry points in a thoracic spine. (B) Burring the cortex of the entry point. (C) and (D) probing the pedicle canal at the proper angle through the vertebral body to the proper depth (E) palpation and (F) screw placement into the canal. [14] 


\section{Entry Point:}

In order to expose the posterior of the vertebrae, soft tissues are removed from facet joints. The appropriate entry point is then marked. The entry point is identified carefully using anatomical landmark on the vertebrae. In upper-left subfigure of Figure 1.5, entry points are specified by two circles.

2. Removing the Cortical Cortex of Pedicle:

Typically, a burr or drill is used to remove the cortical layer of pedicle at the marked entry point.

\section{Channel Creation:}

A straight or curved probe is used in this step. The probe is guided through the pedicle at an angle as depicted in Figure 1.5 (subfigure $\mathrm{C}$ and $\mathrm{D}$ ). The probe is pushed through the pedicle and towards the vertebral body to a maximum depth of 25-40 $\mathrm{mm}$ [11]. The required depth is different for different regions of the spine. The advancement of the probe in this procedure should be smooth and consistent. A sudden change in resistance means that the probe is touching the pedicle wall or the wall of vertebrae body. In such situations, a sudden downward motion can occur if the surgeon continues to apply force to the probe [13]. This phenomenon is clinically known as breach.

Creating a pathway through the pedicle by the free-hand technique is composed of two main degrees of freedom: rotation and linear(translational) progression. Rotating the probe removes the soft cancellous bone and applying force creates linear translational movement along the pedicle axis.

4. Palpation:

Using a pedicle sounding probe, the surgeon verifies that the channeling was carried 
out correctly without causing a breach (subfigure E of Figure 1.5). In cases of cortical cortex penetration, a new channel should be created for screw insertion.

\section{Screw Placement:}

As shown in last subfigure of Figure 1.5, screws of suitable size and length are placed in the pathway. The structure is then ready to be connected to a rod or other instrumentations.

Optimal screw insertion relies on the experience of the surgeon and his capability to differentiate the tactile sensations associated with the different textures in the bone when performing step 3 [2]. During this surgery, due to anatomical reasons, the surgeon has limited visibility to the internal organs or spinal cord. Therefore, he relies dominantly on his tactile proficiency to guide the probe in a right path inside the pedicle. Figure 1.6 shows an exposed posterior of a cadaver's vertebrae.

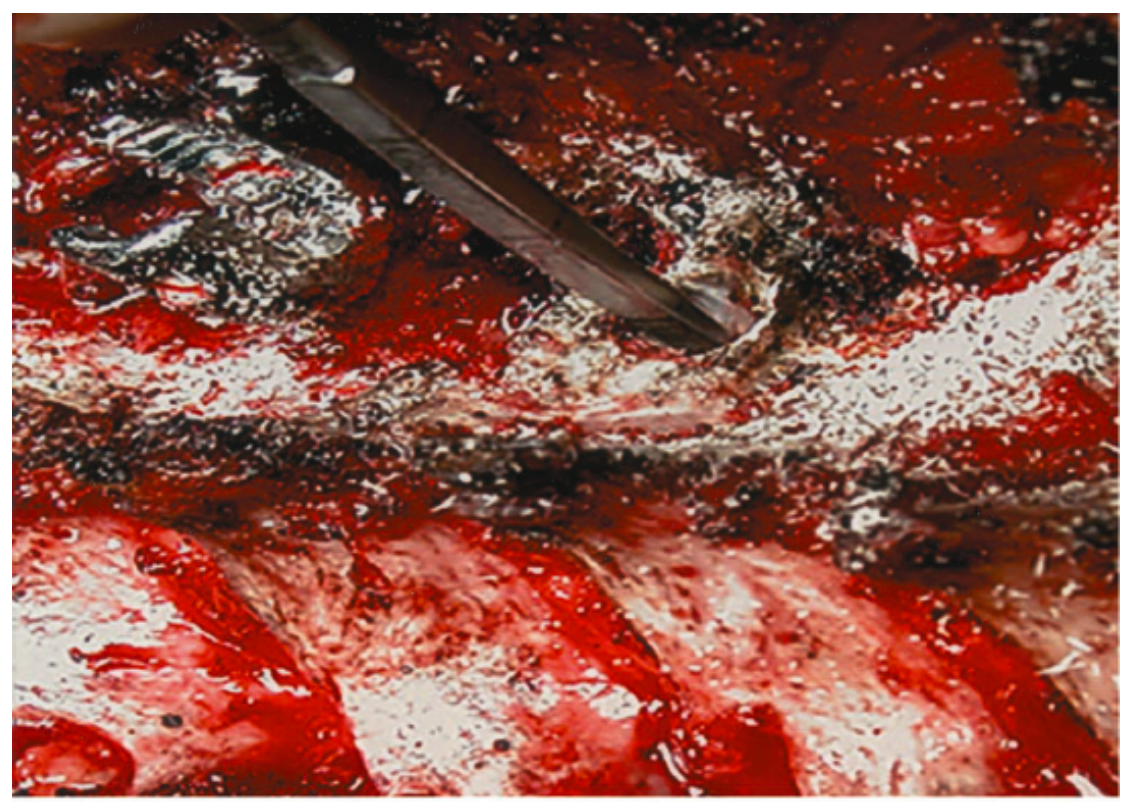

Figure 1.6: Posterior of a cadaver's vertebrae which is exposed for research purpose [15] 


\subsection{Surgery Complications and Reducing Surgical Risks}

Despite the benefits of pedicle screw placement in fixing spinal deformities, complications can occur due to an incorrect entry point, to a surgeon guiding the probe through the pedicle with an incorrect trajectory, and due to a surgeon failing to recognize wall breaches when creating channels. Moreover, in scoliosis, there are further complication due to the abnormal shape of the spine, such as low and inconsistent pedicle size [10]. These vary not only from patient to patient but from pedicle to pedicle. This is why it is so hard to use visualization methods only, even if they are in three-dimensional space.

The complications that pose risks to the comfort and health of the patient are grouped into four main categories according to the local environment of spine: mechanical, neurological, and vascular and visceral. Malpositioned screws and inappropriate instrumentation can lead to problems in mechanical motion. Damaging the nervous system, the spinal cord and nerve roots causes neurological issues. Hemorrhage is the result of injuring vascular structures. Also visceral organs surrounding the spine such as the aorta, lung and the esophagus can be damaged in this surgery $[8,16,7]$.

In a large study of over 1666 patients, it has been revealed that malpositioning has a high error rate of $15.7 \%$ per screw insertion [17]. Another study on cadavers showed an error rate of $15 \%$ for pedicle screw insertion [18]. Although the accuracy of surgeons is not solely due to surgeons experience or lack of experience, studies showed that experienced surgeons have a significantly lower chance of having a medial breach than novices [19]. Figure 1.7 shows a computed tomography (CT) scan of the vertebrae with proper screw insertion and screw malposition. 

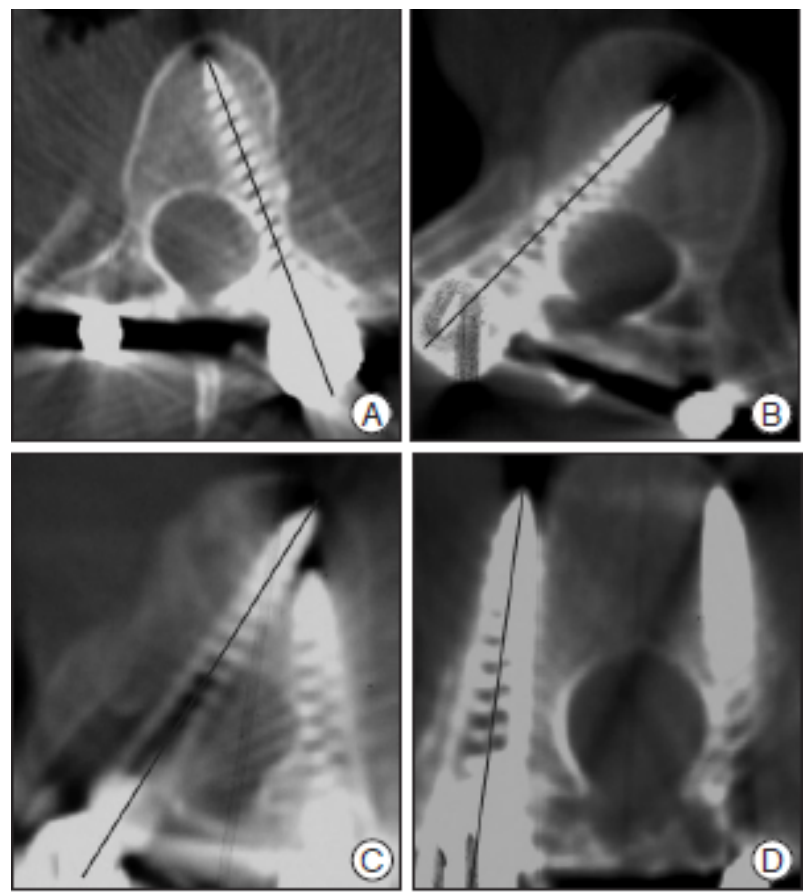

Figure 1.7: Computed tomography (CT) scan of screws inside pedicle. (A) and (B) demonstrate a proper pedicle screw insertion. (C) shows the medial cortical breach and (D) shows a lateral cortical breach [11]

For optimal screw insertion and for reducing the risk of postoperative decompensation, two approaches are under investigation: adding navigational tools to the surgery and improving the medical training for residents.

As a navigation technique, surgeons use image-guided systems such as computed tomography (CT) scans to identify anatomical landmarks prior to or during the surgery. Although navigational tools are helpful for increasing the accuracy of screw placement, it is still a matter of debate as to whether they are necessary for expert surgeons. Operation duration is greatly increased when using medical imaging during the surgery. The surgeon and the patient are also exposed to high levels of radiation. Additionally, there are studies 
that show that accuracy is not significantly improved despite adding considerable extra cost due to the expensive equipment required $[11,10]$. With the steep learning curve in this procedure, improving the education of novice surgeons can involve allowing surgeons to learn and practice the technique in a low-risk environment. Since this surgery relies predominantly on haptic feedback, the differentiation between proper and improper haptic signals should be adequately taught to the residents. The next chapter will investigate the current approaches in the education of surgeons. Among the current approaches, haptic simulators provide the trainee with the safest and most repeatable environment.

The focus of this thesis is on developing a surgery training simulator that imitates the haptic sensations in pedicle screw insertion.

\subsection{Summary}

Information about idiopathic scoliosis, including its epidemiology and treatment are presented in this chapter. Steps in scoliosis surgery which is a common treatment are fully described. Finally, complications of this surgery and the role of surgeons' experience in reducing surgical risks are discussed. 


\section{Chapter 2}

\section{Background}

By providing a structured learning environment, surgical training simulators are perceived to have valuable impact in medical education. This chapter presents a review of the available virtual simulation systems in medicine, and more specifically looks into the concepts of haptics and currently available haptic-related simulators.

\subsection{Medical Training Simulators}

Medical surgery simulators are a recent feasible solution to the challenges of training medical practitioners. Surgical procedures are usually complex and require developing various professional skill sets such as speed, accuracy and hand dexterity. Repeated supervised practice is the key factor to learning and mastering these procedural skills. Traditional surgical training includes the following main categories: supervised practice on live patients, and cadaver, mannequin and animal manipulation and dissection. The method of training under the guidance of an expert mentor and using cadavers has been in use for a long 
time. The alternative option of training on synthetic human organ models is fairly new and has allowed education to be more interactive and engaging. Synthetic materials are used to simulate tissue properties and create realistic anatomical relationships. A summary of advantages and disadvantages of the mentioned non-virtual reality (VR) techniques are shown in Table $2.1[20,21]$. 


\section{Table 2.1: Pros and cons of conventional training approaches}

\begin{tabular}{|c|c|c|}
\hline Method & Advantages & Disadvantages \\
\hline $\begin{array}{l}\text { Apprentice mentoring in } \\
\text { operation room }\end{array}$ & $\begin{array}{l}\text { The most realistic training environment } \\
\text { Apprentice Receives immediate feedback } \\
\text { from the mentor } \\
\text { Exact anatomy }\end{array}$ & $\begin{array}{l}\text { Risk the comfort and safety of the patient } \\
\text { Extend the time and cost of the operation in } \\
\text { order to allow corrections to be made } \\
\text { Training limitation in terms of time and re- } \\
\text { peatability } \\
\text { Difficult to assess the practitioners proficiency } \\
\text { Lack of sufficient access to different case sce- } \\
\text { narios, such as unconventional cases } \\
\text { Ethical issues }\end{array}$ \\
\hline Training on cadaver & $\begin{array}{l}\text { Exact anatomy } \\
\text { Less time restriction for training }\end{array}$ & $\begin{array}{l}\text { Ethical issues } \\
\text { High cost of cadavers } \\
\text { Unrealistic physiological responses due to the } \\
\text { embalming chemicals and lack of blood flow } \\
\text { Training limitation in terms of repeatability } \\
\text { Difficult to assess the practitioners proficiency } \\
\text { Limited anatomic variability due to limited } \\
\text { and uncontrollable supply of cadavers }\end{array}$ \\
\hline Training on mannequin & $\begin{array}{l}\text { Low cost } \\
\text { Time and repeatability constraints are less } \\
\text { than other training methods }\end{array}$ & $\begin{array}{l}\text { Limited realism } \\
\text { Limited anatomic variability }\end{array}$ \\
\hline $\begin{array}{l}\text { Training on synthetic } \\
\text { models }\end{array}$ & $\begin{array}{l}\text { Low cost } \\
\text { High availability and easy portability }\end{array}$ & $\begin{array}{l}\text { Limited realism } \\
\text { Difficult to assess the practitioners proficiency }\end{array}$ \\
\hline Training on animal & $\begin{array}{l}\text { In vivo research (experimentation on a liv- } \\
\text { ing organism) } \\
\text { Allows training with more realistic bone } \\
\text { and body structures }\end{array}$ & $\begin{array}{l}\text { Ethical issues } \\
\text { Not similar to human anatomy }\end{array}$ \\
\hline
\end{tabular}


Virtual reality simulators are a breakthrough alternatives to traditional methods of training [22]. They are computer-based instruments that allow the users to manipulate computer generated objects through special interfaces. Current simulators usually employ visual and touch modalities to replicate the real environment.

With VR simulators, educating practitioners is much safer and easier. It provides the trainee with unlimited practice with no time constraints. Moreover, integrating sensors to the simulator makes it feasible and possible to track the practitioners' proficiency over time. Interactive training systems provide insight into learning processes of trainees and ensure they reach a certain level of proficiency before performing the same procedure in the operating room (OR). There are several researchers investigating skill assessment in training with simulators and its effectiveness compared to conventional approaches [23, 24, 25].

Virtual reality simulators also enable the simulation of various case scenarios using specific data recorded beforehand. The main challenge with VR devices is the realism of the immersion [21]. The fidelity of these technologies is currently the topic of ongoing research.

\subsection{Haptics}

Haptics broadly refers to the technology which uses touch sensation for interaction with objects [26]. This is the only sensory modality that requires a bidirectional flow of information in comparison to other human sensory modalities [27]. As an example, for perceiving the texture of an object, one must rub his finger across the surface laterally until he gains enough information about the object. The human haptic system is stimulated mainly through heat, vibration and pressure and its perception is categorized into two 
sub-modalities: the tactile sense and the kinesthetic sense [28]. Tactile sense conveys information regarding a surface, such as roughness and temperature. Kinesthetic sense, on the other hand, provides information about the force and motion involved when manipulating an object.

Interaction with objects can be done through humans and/or machines and the object itself can be real and/or virtual. Sensing the shape of a cup is a real haptic manipulation. A haptic interaction can be created between a user and a virtual object through a haptic device that exchanges information bidirectionally, from and toward the user.

Therefore, haptic technology involves human haptics, machine haptics and computer haptics [27]. As previously discussed, human haptics refers to the human haptic sensory system. Machine haptics deals with developing haptic interfaces that enable human interactions with virtual environments. This component stimulates the human perception system by creating mechanical signals. Commercial haptic interface devices have appeared for several applications in various forms. They are often robot manipulators and basically split into two main categories: impedance controlled devices and admittance controlled devices. In the former, the device measures the operator's displacement and displays haptic force feedback. The latter, however, has the inverse concept of the former.

Haptic interfaces are sometimes used for tactile interactions. Touch Master in 1993 and CyberTouch glove in 1995 are among the first commercial tactile feedback interfaces. The first force feedback manipulator was used by Goertz in a teleoperated system to handle radioactive substances in the early 1950's [29]. Common commercial force feedback devices used in laboratories includes PHANToM family ${ }^{1}$ and Novint Falcon ${ }^{2}$. Although, these devices offer portability and affordability, they cannot exert high output force, typically

\footnotetext{
${ }^{1} \mathrm{http}: / /$ www.dentsable.com/products-haptic-devices.htm

${ }^{2}$ http://www.novint.com/index.php/novintfalcon
} 
less than $10 N[21]$.

The other aspect of VR simulators that deals with modeling virtual environments(VE) and haptic rendering is called computer haptics. Haptic rendering includes collision detection, force feedback calculations and control schemes. VE is a computer simulated environment that replicates real phyical objects and sensory modalities. Simulation environments formerly incorporated mass-spring techniques for modeling force feedback. Recent works, however, focus more on employing finite-element approaches. This method, however, has significantly higher computational cost [30].

Haptics, when combined with vision, usually creates more realistic virtual effects than by haptics itself. These two sensory modalities have different properties. In vision, recognition starts with the general overview of the object. In haptics, however, recognition starts locally and then the perception of the whole object is constructed [27]. Vision technology is more developed and complex than haptics. It is generally reported that visual applications alone are superior to haptic applications. Therefore, most haptic applications are coupled with visual feedback. However, there are certain tasks that are mainly haptic in nature. This is the case for visually impaired applications or in tasks where even in the real environment, vision is rarely incorporated. For example, in the procedure of channeling the pedicle, vision is very limited. Therefore haptic cues play a key role in the task. In the absence of visual feedback, it is often difficult to recognize the nature of an object [31] and therefore accurately simulated haptic feedback is a key factor to reduce the performance error [32]. 


\subsection{Haptic Training Simulators for Medical Applica- tions}

Haptics and haptic simulations are used in the field of medicine, especially in training. Haptic platforms are widely used for palpation; the trainee is trained to feel virtual anatomic features, such as tumors, beneath the virtual skin [33]. Some other simulators incorporate models of needles, tissues and bone to replicate suturing [34]. A 2D/3D image of the organ is usually reconstructed from CT or MRI images and is displayed to the practitioner. Many simulators have been developed for laparoscopic, enodscopic and arthroscopic surgeries [21]. While most of the available platforms are designed for interaction with soft tissues, there are a few works developed for surgery on bony structures. Steinberg et al developed a dental training simulator that uses the PHANToM for haptic feedback as well as 3D-VR graphics for the visual system [35]. Some researchers have studied haptic simulation of bone-sawing, boring, milling and drilling. An example is a bone surgery simulator designed by Morris et al in a visuo-haptic environment that replicates mandibular surgery and temporal bone surgery [30]. For the purpose of emulating orthognathic surgery, a simulator with capability of practising cutting, separation and rearranging bone was developed in [36].

\subsection{Haptic Training Simulators for Pedicle Screw In- sertion}

Among conventional methods of surgical education, cadaver lab training and apprenticeship are the most common techniques for pedicle screw insertion training. As discussed 
earlier, these methods come with downsides. A new way of addressing these limitations include computer simulations, which employ haptic and/or visual feedback in the virtual environment to replicate the real procedure.

A group in Singapore created a simulator for vertebroplasty surgery training. This type of surgery treats compression fractures of the vertebrae by injecting cement into the vertebrae. Their simulator provide the force feedback by a delta haptic device ${ }^{1}$ and $\mathrm{Cy}-$ berGrasp glove ${ }^{2}$, and visual feedback by a 3D graphical interface [37]. They also presented biomechanical models for needle insertion into the bone [38].

A problem with many of the available platforms is that they fail to create realistic effects due to device limitations. For instance, the haptic feedback related to a spine biopsy simulator remained limited to interactions with soft tissues since the haptic device being used was unable to provide high realistic force peaks [39, 40].

For the procedure of pedicle screw placement in scoliosis surgery, the freehand method is commonly employed [41]. As with many other surgery procedures, it is essential that surgeons become skilled in the free hand technique. The errors in channeling lead to loose and weak pathways for placing screws, resulting in complications after surgery. Additionally, due to abnormal spinal morphology in this disorder, specialists often encounter complicated cases. Therefore, a high level of accuracy is required for spinal fusion procedures. A study [19] has shown that the percentage of proper screw placement is higher for experienced surgeons .

Most available works focus on the visual aspects of the surgical education. The VR platform of Klein et al is a recent example [42]. They implemented a computer simulation of a three-dimensional CT-based model of the spine and allowed the novice surgeons to

\footnotetext{
${ }^{1}$ http://cs.stanford.edu/people/conti/delta.html

${ }^{2}$ http://www.cyberglovesystems.com/products/cybergrasp/overview
} 
practise the procedure with various sizes of screws. The trainee can manipulate pedicle screws and place them at distinct entry points with different orientations. One is also able to have a better sense of the trajectory and the depth of screw by manipulating the image. The trainee's performance then can be assessed by measuring screws position and angles.

In an earlier study in [43], Eftekhar et al. designed a free, downloadable computer program which allows the user to insert virtual screws into a two dimensional vertebrae while receiving visual feedback. The program allows users to work in different regions of vertebrae. Rambani et al. also created a computer-assisted training device for pedicle screw fixation and then tracked the improvement of training surgeons before and after working with it. The device involves interacting with a computer image of vertebrae created from scanned medical images [44].

For pedicle screw insertion in idiopathic scoliosis correction surgery, the screw hole is usually shaped by a cannulation probe. Most virtual reality simulators for bone tissue procedures, however, involve power drill simulations. Schmidt's vertebrae simulator, for example, has a virtual model of drill movement which is rendered visually with real time volume techniques [45]. More recently, a research group created a visual and haptic platform utilizing ImmersiveTouch (an augmented reality and haptics workstation) and a head-and-hand-tracked high resolution toolkit [46]. The system is programmed such that the practitioner can use a power drill for boring the spine in virtual reality. Fluoroscopic images are also available for the initial phase of training. Subsequently, training conditions can become more constrained by omitting this navigational tool. The simulator trains the practitioner on a predefined trajectory with reactionary forces applied by the haptic device.

There is also research investigating navigation tools as alternatives that aid proper pedicle screw placement. These real-time bone-imaging techniques are fully explained and 
discussed in [41]. They includes techniques such as: fluoroscopic imaging and CT-guided computer-assisted surgery and ultrasonic-guided screw insertion. However, some argue that the image-guided systems and assisted technologies are luxury tools that most expert spine surgeon would not need in real situations [10].

The importance of manual probe channeling, usage of a cannulation probe, and the demand for high-fidelity haptic feedback for simulating the procedure of pedicle screw insertion contributed to the idea of developing a customized simulator for pedicle screw placement in idiopathic scoliosis surgery.

\subsection{A Summary on the Progress of the Project by Previous Researcher}

As discussed earlier, a vital step in the free hand technique for pedicle screw insertion involves the use of a probe to make a channel through the vertebrae. Regina Leung started the first phase of haptic training simulator for spine surgery. This haptic simulator would emulate what the surgeon feels in this surgery while using the probe.

Her work [47] involved creating a 1 degree of freedom haptic device for the simulation of probe rotation during the pedicle screw surgery. A pilot study was then conducted using the haptic simulator. Five surgeons of varying experience levels used the simulator set at three bone density settings (normal, low, and high density) and were able to change various parameters in order to tune the simulator to what the surgeon felt was the most realistic representation of the haptic sensations for three levels of bone density. The surgeons were also asked to rate whether they believed the simulation to be a close representation of using a probe on an actual vertebrae, and whether they believed the simulation could be 
used in teaching. The surgeons all gave favorable feedback on both questions.

Her work, however, requires a correction in the implementation of the rotary effects. There was another limitation in terms of sample size. Only five surgeons were recruited for the clinical tuning and the simulator was designed to only emulate the rotary effects of the procedure. The objective of the present work is to do the hardware and software development with an aim to extend the previous work by adding the linear degree of freedom, coupling the 2 degrees of freedom and conducting clinical studies with a larger group of the medical experts.

\subsection{Summary}

In this chapter, the role of virtual reality simulators to augment the conventional surgical training is discussed. An introduction to the haptic technology and current available haptic simulators are presented. Current available Simulators that are designed for emulating pedicle screw insertion involve using drilling tools or they have focused on visual aspect of the surgery. Our haptic simulator is designed for manual surgery and is capable of simulating high forces created in bone interaction. The chapter ends with a summary of the work that is accomplished by a previous researcher. 


\section{Chapter 3}

\section{Biomechanical Characteristics of the Surgical Procedure}

The pedicle is composed of two types of tissues: cancellous bone (also called spongy or trabecular bone) and cortical bone (also called compact bone). Cortical bone is the harder tissue that forms the outer layer of the pedicle wall. Cancellous bone, on the other hand, is soft and has a low density structure and it is located within the interior of the pedicle. In Figure 1.4, a cross-sectional image of vertebrae is shown along with the tissues discussed.

The procedure of probe channeling in pedicle screw insertion can be split into two degrees of freedom: rotation and linear progression. Expert surgeons describe probe rotation through cancellous bone as a task that causes vibrations while clearing away the bone lattice in this region. It also creates the haptic feeling of going over a series of bumps, similar to scratching a match across a surface. During probe rotation across the cortical bone, however, viscous friction is the dominant sensation [47].

Linear progression through the pedicle displays a slightly different behavior. While the 
surgeon is clearing away the lattice layer of the cancellous bone and pushing downward at the same time, linear motion feels like going over small bumps in a smooth and consistent manner. This sensation is likened to the feeling created when driving a piece of metal along a tube whose interior wall is made of coarse sand paper. The surgeon should continue channeling the probe into the body until the depth is deep enough to place the screws. A depth of 30-40 mm is normal for most cases. As the spine extends from lower thoracic region towards higher thoracic region, the size of vertebrae decreases and therefore less in-depth progression is needed [15].

A perforation of the pedicle wall is an adverse event which can occur in this surgical procedure. As illustrated in Figure 3.1, the breakthrough can occur in four primary ways:

1. Breaching through the inner pedicle wall: The probe moves from inside of the pedicle into the spinal cavity.

2. Breaching through the outer pedicle wall: The probe moves from inside of the pedicle into the body cavity.

3. Breaching through the bottom of the pedicle wall: In lateral view, the probe moves from inside of the pedicle to the bottom of the pedicle.

4. Breaching through the vertebral body wall: The probe moves from within the body to the body cavity. 

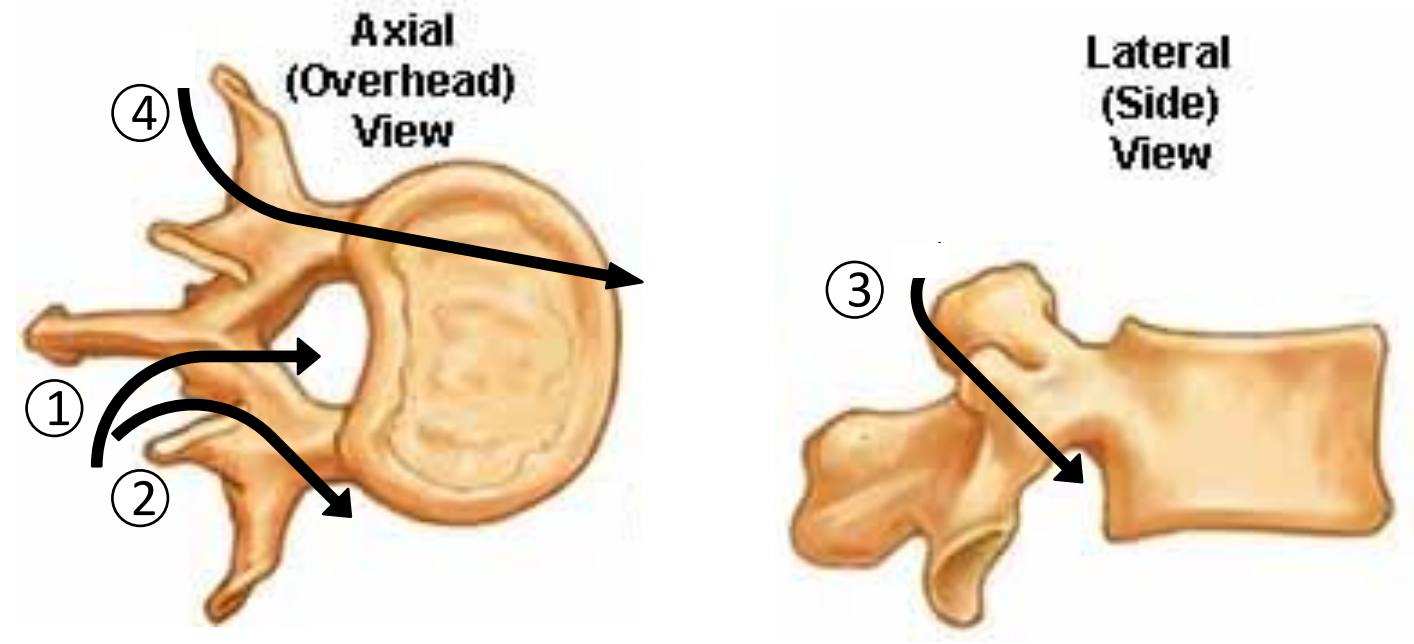

Figure 3.1: Possible types of breakthrough [5]

The first three breaches occur due to an incorrect initial probe trajectory while the last one occurs when the surgeon at some point feels a high resistance to the probe but continues to apply pressure. This high force results in a dramatic perforation. A sensation of high resistance force can help surgeons identify the situation.

The size of the bumps and significance of the breach depends on several factors: the type of deformity, the age of the patient, the sex, the region of the vertebral column, bone density and pedicle size. Some of these conditions are known to surgeon prior to the procedure. However, each case has a unique combination of the factors which make it distinct and complicated, especially for a novice. 


\subsection{Scenarios Considered for Simulation}

Based on feedback from expert surgeons, for the linear degree of freedom, probe channeling is a combination of two system effects: linear motion and breach effects. Linear motion is the regular effect that any procedure contains and breach occurs in the cases described in previous section. As discussed earlier, anatomical characteristics differ between different patients and also within different vertebrae regions of a patient. Instead of creating several simulation effects for different cases, a more effective procedure has been devised. For the initial prototype, we classified the effects into main groups and then investigated ways to simulate them. After open discussion with orthopedic surgeons, similar to the previous study, we classified the different anatomical scenarios based on the common situations encountered in spinal surgery: low density vertebrae with low pedicle size, normal density vertebrae with normal pedicle size and high density vertebrae with high pedicle size. Low density vertebrae are common in elderly patients and patients with osteoporosis and high density vertebrae are encountered in young patients, while normal vertebrae with normal size and normal bone density are seen in a healthy patient. We then attempted to create the torque and force profile corresponding to each anatomical scenario.

Although the proposed haptic model in this study is capable of simulating all three cases, for the purpose of this thesis, clinical testing was only performed for the normal scenario across all recruited surgeons, and the low scenario testing protocol was carried out for only a few participants. This decision was made to keep the test duration short in order to avoid bias caused by fatigue. Also, it will be fully explained later in next chapter that due to the hardware limitation, simulation of breach effects is postponed to further investigation. 


\subsection{Summary}

Haptic sensations that are felt in the probe channeling through the pedicle are discussed in this chapter. Probe channeling consists of two degrees of freedom: rotation and linear progression. Breach is an adverse effect that can occur if the surgeon fails to recognize abnormal forces in linear progression. The chapter concludes with describing the testing scenario which is the simulation of normal bone. 


\section{Chapter 4}

\section{Methodology}

\subsection{Research Methodology}

The cyclical iterative design scheme introduced by Thomke [48] was used for the development of the haptic training simulator. This method, in brief, is an iteration of design-buildtest-analyze. This design process allows for refinement in response to mistakes. Steps for this research problem are shown in Figure 4.1 


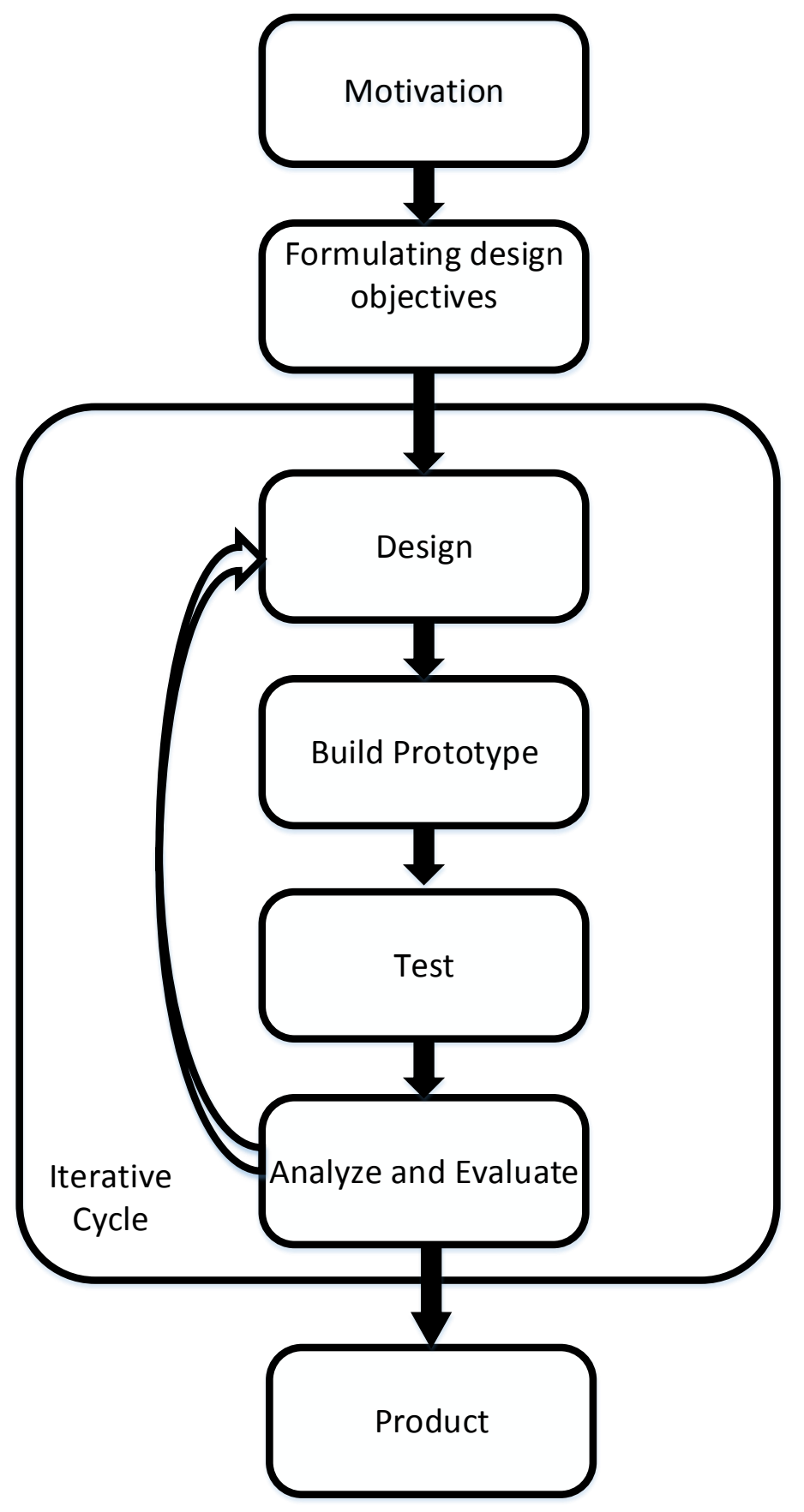

Figure 4.1: Research model 


\section{Motivation:}

First, the clinical problem is studied and the background is fully conceptualized. Literature reviews are done about probe channeling for pedicle screw insertions in scoliosis spine disorder. Haptic sensation discrimination was recognized as an essential component of training the novice orthopedic surgeons.

2. Formulating design objectives:

In the second step, the design requirement for developing the haptic simulator is devised. The design requirement for simulating the biomedical characteristics of the procedure is thoroughly defined. Present work looks at simulating linear motion and integrating it with rotary motion.

3. Design:

This is the starting point of the four-step iterative cycle. Based on the design requirement, hardware, software and interfacing components of the simulator are specified.

4. Build prototype:

In the second step of the cycle, the mechanical apparatus is built and the haptic model and the control algorithm are implemented through the software.

5. Test:

In the third step of the cycle, the system and the proposed models are tested with the orthopedic expert surgeons in a pilot study and feedback is obtained regarding the feasibility of simulating the haptic sensations. The experiment is also done for the purpose of clinical tuning.

6. Analyze and evaluate: 
In the final step of the iterative cycle, the results are analyzed. If the test results are not satisfactory, the iteration continues and modification is applied on the unsuccessful part of the design. This modification could be applied on the simulation strategy, the choice of hardware, or any unconsidered specifics. Otherwise, iteration is stopped and one proceeds to step number 7 .

Several iteration cycles are carried out in order to get the optimum performance for the system.

7. Product:

Once the iteration cycle is completed and the results in the sixth step are satisfactory, the prototype is ready for deployment.

\subsection{Research and Design Objectives}

The main objective of this research is to develop the second stage of the haptic training simulator for pedicle screw insertions in pediatric scoliosis surgery. The first phase of the project involved developing the first rotational degree of freedom (DOF) [47]. This thesis adds the linear degree of freedom to the simulator.

Similar to the first phase of the project, surgical observation and feedback from surgeons was used in creating the haptic sensations. Haptic model parameters were then clinically tuned by conducting a user study with a group of spine surgeons. 


\subsection{Control Structure}

The general control structure that is used in this work is shown in Figure 4.2. It includes the rotary stage control scheme, linear stage control scheme and the scheme used for coupling the rotary and linear stage.

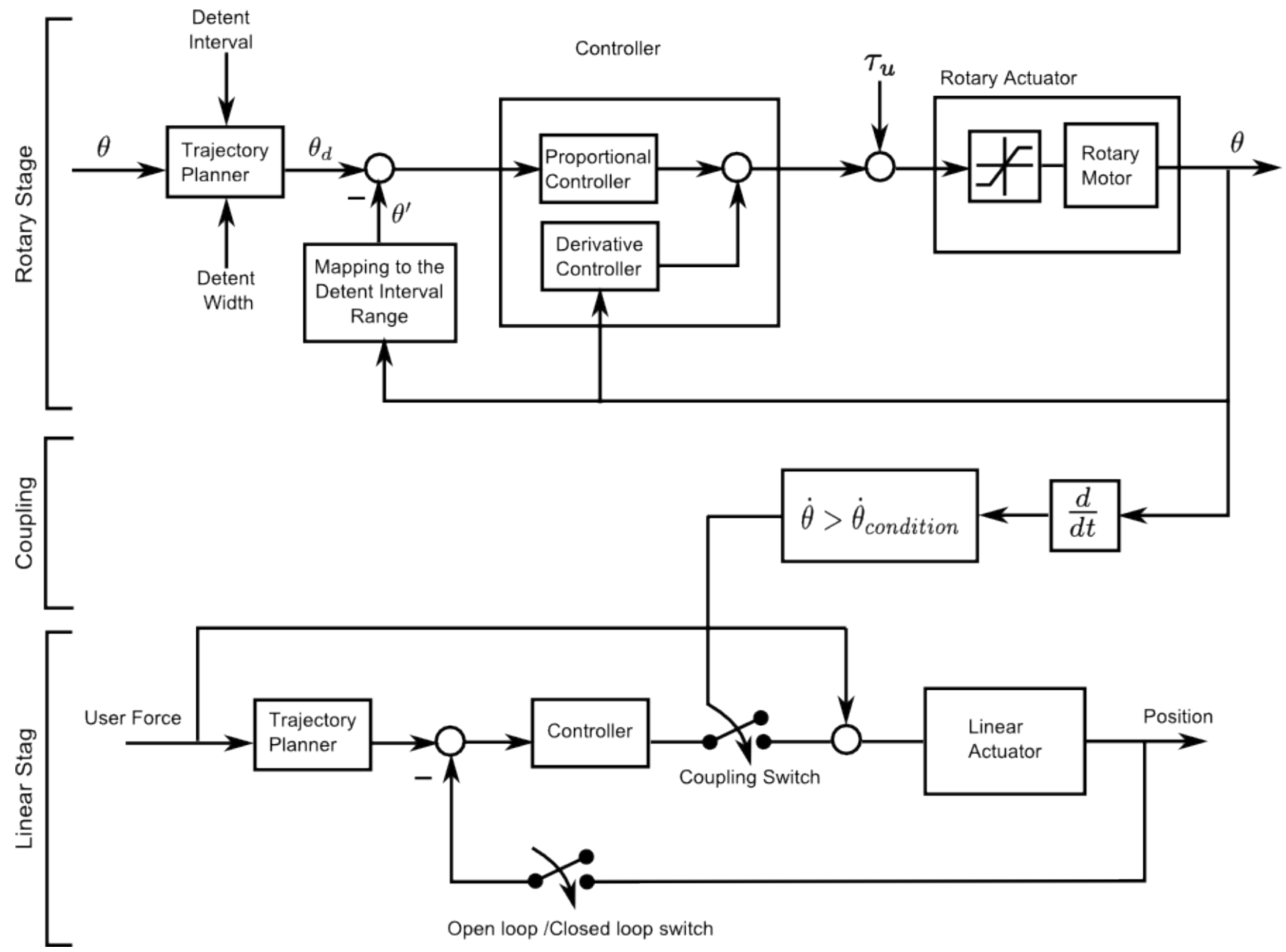

Figure 4.2: General control diagram of the haptic training simulator 
What is presented in the next section is what is used as the haptic model for the rotary stage in the current work. A slight modification was applied to the implementation of the effects explained in the previous work in [47]. The following section will discuss two potential control strategies that are suitable for implementing the linear haptic effect. Subsequently, the integration between the re-designed rotary haptic model and the linear haptic model will be discussed.

\subsubsection{Summary of the Rotary Stage Haptic Model}

The simulation model that was proposed in [47] consists of two main haptic effects: vibration effects and viscous friction effects. Vibration is modeled as a series of bumps in rotation and a time-varying proportional controller is used to simulate it. Viscous friction effects are generated by a derivative controller and the effects are felt as resistive torque which increases relative to the speed of rotation. The block diagram for the rotary stage is shown in Figure 4.2

In the block diagram, $\theta_{d}$ is the desired angular position and is generated using the trajectory planner block. This block uses Equation 4.1 that is a function of two parameters: detent interval and detent width (which are indicated by $\theta_{i}$ and $\theta_{w}$, respectively).

$$
\theta_{d}= \begin{cases}\theta^{\prime}, & \text { if } \theta^{\prime}<\theta_{L} \\ \theta_{L}, & \text { if } \theta_{L}<\theta^{\prime}<\frac{\theta_{i}}{2} \\ \theta_{R}, & \text { if } \frac{\theta_{i}}{2}<\theta^{\prime}<\theta_{R} \\ \theta^{\prime}, & \text { if } \theta^{\prime}>\theta_{R}\end{cases}
$$

where $\theta^{\prime}$ is the remainder obtained if $\theta$ is divided by $\theta_{i}$. $\theta_{L}$ and $\theta_{R}$ are also computed 
as follows:

$$
\theta_{L}=\frac{\theta_{i}-\theta_{w}}{2}, \quad \theta_{R}=\frac{\theta_{i}+\theta_{w}}{2}
$$

The response torque is calculated using a PD controller

$$
\tau_{c}(t)=k_{p}\left(\theta_{d}-\theta^{\prime}\right)-k_{d} \dot{\theta}
$$

The proportional component of the controller creates series of detents in the probe position as shown in Figure 4.3. When the position of the probe lies in the first and last case in Equation 4.1, the created torque is zero. As the probe position moves away from $\theta_{L}$, the controller creates a incremental resistive torque which feels like the compression of a spring. When the probe passes the midpoint of the detent interval and moves toward $\theta_{R}$, the direction of the torque changes and the magnitude of it starts decreasing. The sensation is similar to the feeling of of an extended spring returning to its equilibrium point. The magnitude of the detent is controlled by the proportional gain in the controller. The detent interval, detent width and the proportional gain are the parameters which vary the vibration.

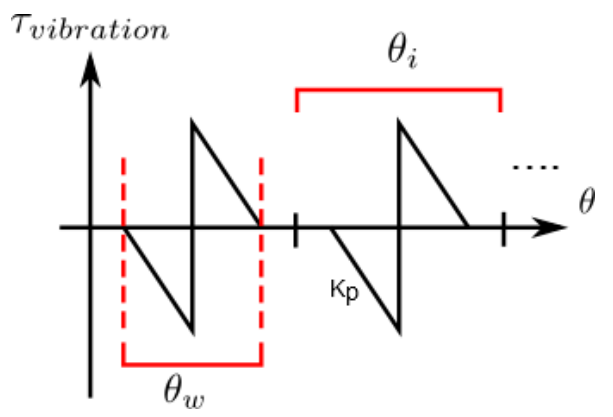

Figure 4.3: Proportional component of the rotary stage control signal 
The derivative part of the controller is responsible for creating viscous friction effects. This sensation is likened to rotating the probe within a viscous material. As the probe is rotated faster, the friction sensation is increased. The $k_{d}$ gain is the parameter that determines the magnitude of friction effect.

All four haptic model parameters (detent interval, detent width, detent magnitude, viscous friction coefficient) are then used in the clinical testing to be tuned to what felt most realistic according to the actual surgical scenario.

It should be noted that the vibration effects are implemented with a slight change from

the previous work. In Leung's thesis [47], the ratio of detent width to detent interval was used for tuning. In this work, however, detent width is tuned independently. In addition, the angular position that was fed back to the proportional controller in Figure 4.2 was not properly mapped to the detent interval range in Leung's work. As a result, vibration effects were not presented to the user as intended. For all sets of parameters, detents were felt as more compressed and closer than what they should have been. This issue has been resolved in the present work.

\subsection{Potential Control Structures for Simulation of the Linear Haptic Effects}

The second stage of this project is simulating the linear dynamics. These linear haptic effects are different in concept and implementation than the rotary haptic effects. In order to simulate the biomechanical charectristics of the probe channeling through the pedicle, an actuator is required that provides a translation degree of freedom.

Regardless of the choice of hardware, the device should replicate the vibration and re- 
sistance sensation felt as the probe proceeds through the pedicle. It is important, however, that the actuator be able to handle the weight of the rotary stage and not move when it is not powered. Another key factor is that the actuator must be capable of supplying very large forces. This is important for simulating breach effects. Given these constraints, the choice of actuator becomes limited to pneumatic actuators, hydraulic actuators, or DC electrical motors. Pneumatic and hydraulic actuators are very fast and powerful but are generally large and expensive. They also require noisy and high maintenance hydraulic or pneumatic compressors. Therefore, electrical geared motors are more feasible in this project. Due to these limitations, the design of the controller for simulation of the linear haptic effects is nontrivial.

In haptic applications, one of the conventional techniques is impedance control. Impedance control is often used for achieving desired dynamic interaction between a robot end-effector and the environment in the design of a virtual reality environment. In this approach, the interaction force is controlled by controlling the actuators impedance which is the measure of the actuators motion when subjected to a force. For example, an actuator with zero impedance is completely backdrivable and freely manipulated. An actuator with infinite impedance, conversely, implies a stiff environment.

Devices that use impedance control are often highly backdrivable and usually have a light structure with low friction mechanisms [49]. If the actuator is geared or highly nonbackdrivable, the total inertia felt at the end-effector is increased. This property makes it difficult to control the robot to behave with a desired impedance [50]. Thus, the choice of hardware is our principal barrier in employing an impedance control approach.

In the following sections, two control strategies will be presented that are potentially applicable for simulating haptic effects in the linear stage. These techniques follow the general block diagram shown in Figure 4.2. One strategy employs closed loop PID control 
technique when the switch in the feedback path is closed. The other strategy uses an open loop control scheme when the switch is open. In the simulation of linear progression, both simulate the vibration by making step-wise motions whose progression speed is also a control variable that determine the sense of resistance opposing the user force. Breach simulations involve simulating the dropping of the probe with a certain displacement that occurs in a short time at a certain threshold in user force.

The effectiveness of these approaches are then evaluated by conducting pilot studies with expert surgeons. Details of such studies will be presented in the following chapters.

\subsubsection{Closed Loop PID Control}

In Figure 4.2, when the switch in the feedback path of the linear stage's block diagram is closed, the control loop becomes closed. A trajectory planner is used to generate the desired reference trajectory and a PID controller is employed for driving the motor to follow the trajectory. A detailed block diagram for this control approach is shown in Figure 4.4

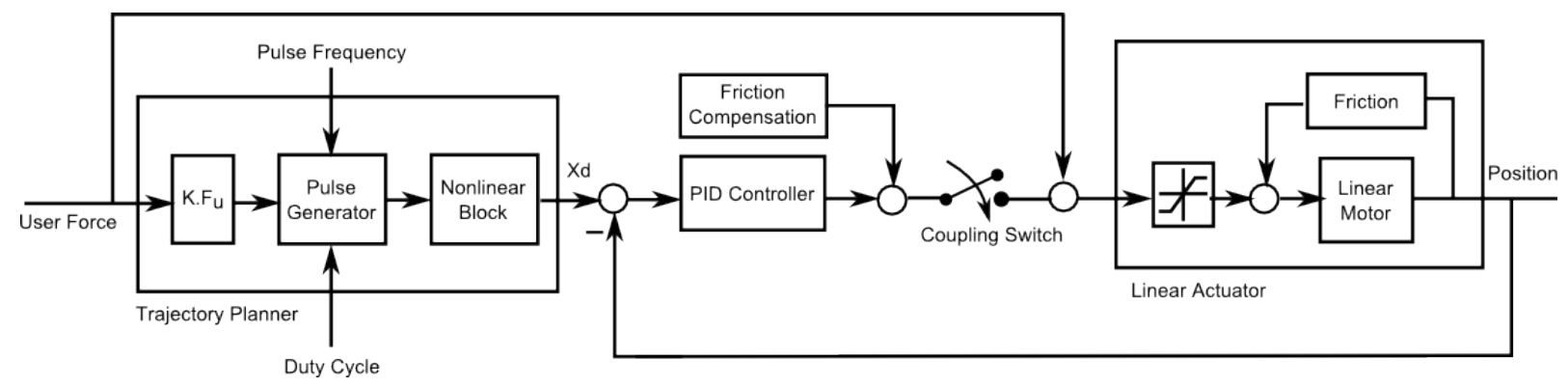

Figure 4.4: Block diagram of the PID control scheme

PID controllers are widely used in the control of robots due to their simplicity and acceptable performance for a wide variety of actuators. Accurate modeling and identification 
of the system is a key step prior to the design of a high performance controller. The dynamical behavior of the linear motor can be approximately modeled by linear relationships. For a motor with friction, a cancellation term should be devised for the friction force. The ultimate goal is to be able to perform precise position control of the linear actuator.

\subsubsection{Linear Progression Simulation}

As shown in Figure 4.4, the trajectory planner uses three inputs to generate the desired trajectory. First, the user force is scaled by a factor of $k$ and then is converted to a series of pulses. Gain $k$ determines how much force the user should put on the probe to get motion. Pulse frequency and duty cycle are also tuning variables that can change the vibration sensation. Output signals of the pulse generator are then passed through a nonlinear block. In this block the current signal is added with its one-sample-delayed signal (see Figure 4.5). Note that when the user keeps applying force on the probe, displacement is always expected. Therefore, this nonlinear block is considered to update the reference trajectory.

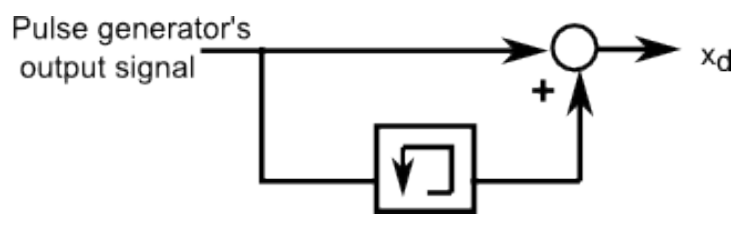

Figure 4.5: Inside the nonlinear block

For the selected hardware, a motor model needs to be proposed and identification techniques should be employed to find the model parameters. Friction compensation and PID control parameters will be designed to meet the requirements. For the control design, it is important to achieve quick responses with small overshoot for any changes in the 
reference. Details of a such design and the corresponding computations are discussed in the next chapter.

\subsubsection{Breach Simulation}

To simulate breach effects, the same block diagram is used with the change in the implementation of the trajectory planner that is shown in Figure 4.6. Desired displacement of the drop is one of the inputs. This block diagram also takes the user force and compares it with a tunable force threshold. When the condition is satisfied, a step with the size of desired displacement is injected in the reference trajectory. The nonlinear block which contains the $\mathrm{ZOH}$ and the summation operator adds this change to the trajectory. The condition remains idle for a short time so as to avoid multiple true conditions that are potential in the presence of noisy signals.

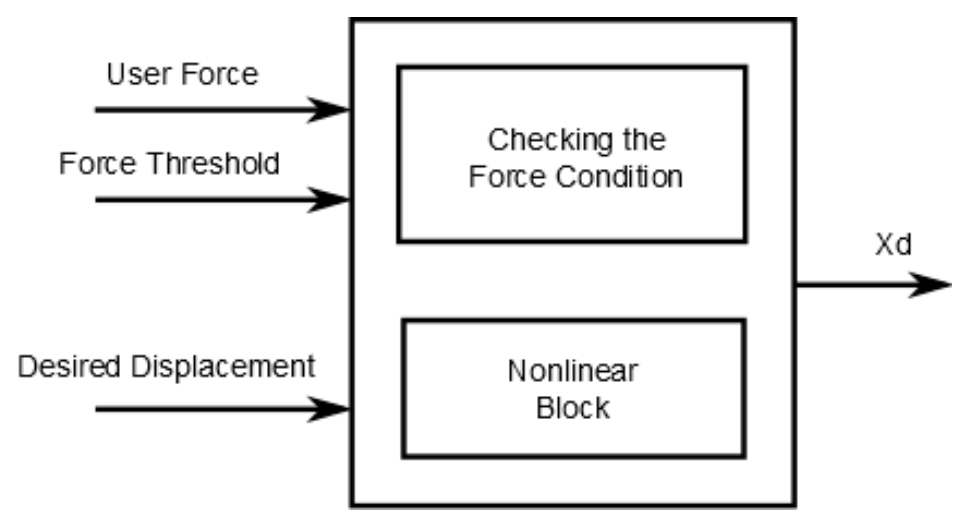

Figure 4.6: Trajectory planner used for the simulation of breach effects in closed loop PID control 


\subsubsection{Open Loop Control}

The control loop becomes open when the switch is open in the feedback path of the linear stage's block diagram in Figure 4.2. The distance is no longer the main focus of this control; instead, the speed of movement is controlled in an open loop manner. The trajectory planner and the controller block are merged into one block as shown in Figure 4.7.

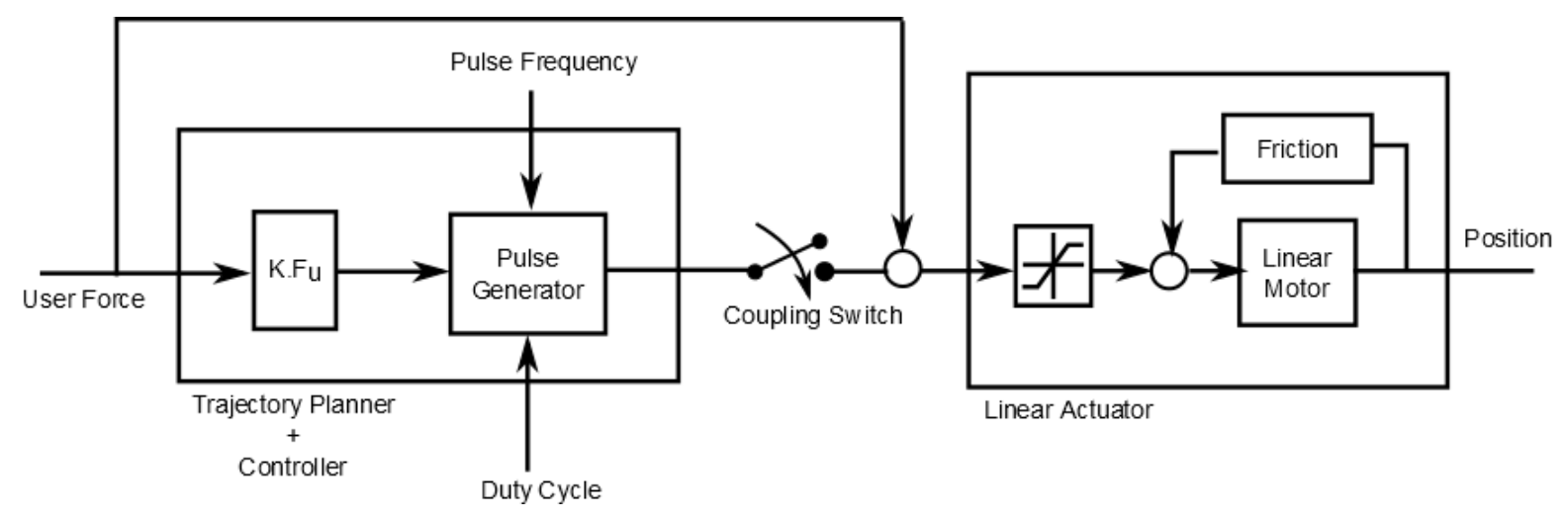

Figure 4.7: Block diagram of the open loop control scheme

\subsubsection{Linear Progression Simulation}

For the simulation of linear progression, the goal is to create piece-wise motion in the presence of vertical forces. This can be achieved by simply feeding the actuator with a series of pulses. User force is measured and scaled by a factor of $k$. This gain specifies how resistive the motion will be. The signal is then mapped to a series of pulses as shown in Figure 4.8. The frequency and the duty cycle of the pulses determine the vibration sensation felt by the user. If the actuator has friction inside the motor, part of this control signal is used to overcome friction and the rest of control signal moves the actuator. 


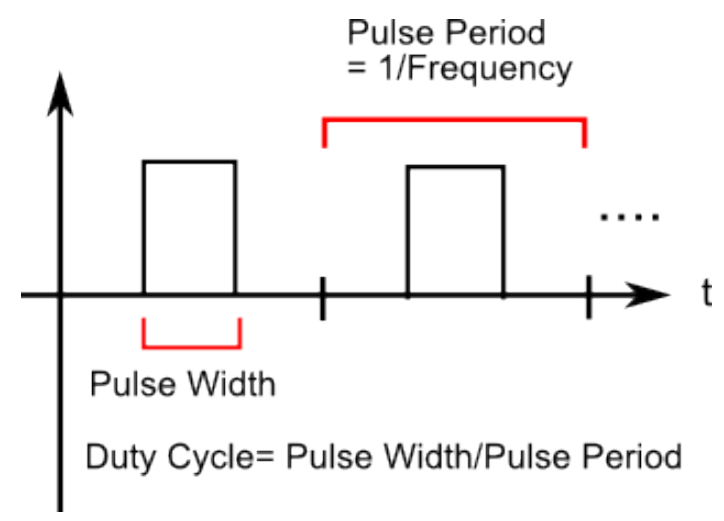

Figure 4.8: Frequency and duty cycle of pulse series

\subsubsection{Breach Simulation}

The same block diagram in Figure 4.7 is used for the breach simulation scheme. The first block, that is the combined effect of the trajectory planner and controller block, is replaced with the block in Figure 4.9 .

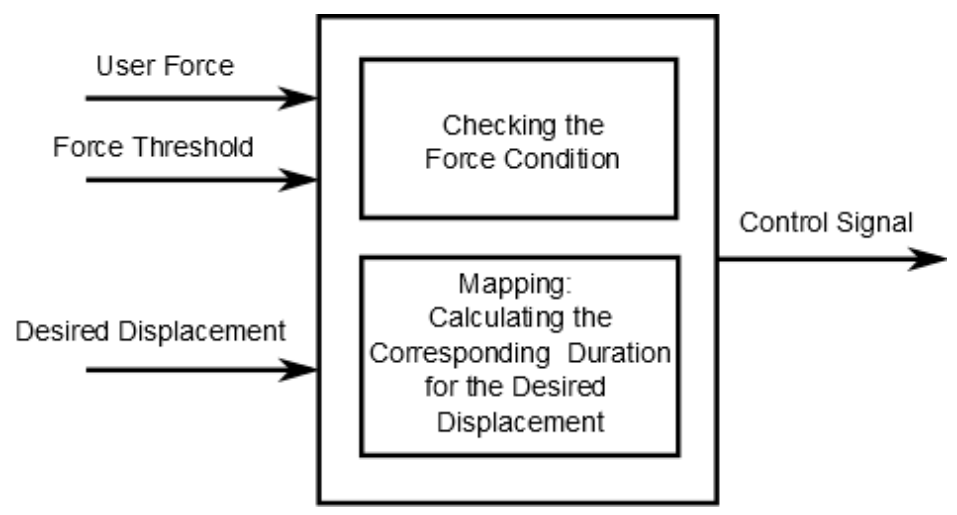

Figure 4.9: Trajectory planner used for the simulation of breach effects in open loop control

Since breach is a very quick event, it is very important to use the maximum speed of the system. When an actuator is fed with a pulse of maximum input, the actuator moves 
and the displacement is increased as the duration of the pulse increases. The relation between the pulse width and the achieved displacement can be approximated with linear regression. The block gets the desired breach displacement and uses the mapping relation to approximate the pulse duration. Similar to the technique used in the closed loop technique, it is continuously checked whether the user force reaches the desired force threshold. When the condition is true, a pulse with the certain width is applied to the actuator. The condition remains idle for a short time so to avoid multiple true conditions that are potential in the presence of noisy signals.

Details regarding the relation between the pulse width and the achieved displacement are presented in the next chapter when the hardware is described.

\subsection{Coupling Rotary Haptic Effects with Linear Hap- tic Effects}

Through surgical observations and discussion with expert surgeons, it was found that rotation is also a key factor in progressing through the pedicle. In other words, surgeons rotate and push the probe simultaneously. Rotating while pushing eases clearance of the lattice layer and make the pathway clear for the screws. Another benefit of rotating the probe is that the surgeon can then control the speed of progression, as opposed to when the surgeon just pushes along the axis with a higher force. The latter has higher risk of breaching the pedicle.

To simulate this, angular velocity of the probe rotation is used in a condition that determines how subtle the rotation should be to allow for linear progression. As shown earlier in Figure 4.2, it is verified through a comparison block whether the angular velocity 
is larger than a pre-determined velocity. This condition controls the function of the coupling switch that passes the linear stage control signal to the actuator. The velocity condition is captured by conducting pilot studies and by receiving surgeons' feedback.

\subsection{Summary}

Design scheme and design objectives of this research are presented in this chapter. The control structure for the rotary stage and linear stage are discussed as well as the proposed technique to couple these two stages. The rotary stage haptic model that was developed in the previous work along with its required modification are summarized. Finally, potential methods for the simulation of linear stage haptic effects are presented. 


\section{Chapter 5}

\section{Experimental Platform}

The experimental platform includes hardware, software, and the interface between them. In this chapter, the development of the haptic simulator is discussed. The rotary stage of the robot that was built and tested in [47] is extended to provide functionality for the linear degree of freedom. The platform components will be fully presented in the following sections. Subsequently, the proposed control techniques that were introduced in the previous chapter are employed for the developed haptic robotic system.

\subsection{The Rotary Stage Platform}

The hardware and software required to simulate the rotational degree of freedom was designed and developed in the first phase of the project by Regina Leung [47]. As seen in Figure 5.1, the mechanical component included a fabricated probe handle which was coupled to the shaft of a non-geared DC motor. The probe was machined so that it would feel and operate like the actual probe used by the surgeon. The operator holds the probe 
in his hand with the palm on top and fingers around the probe. He then rotates the probe back and forth. The probe was intended to have no mechanical play. An encoder was connected to the motor to enable measuring of the angular position.

The hardware was interfaced to the computer through the breakout board and data acquisition system (DAQ). The rotary stage haptic model (see Section 4.3.1) was implemented in the computer in the Matlab/Simulink programming environment. The output signals of the haptic simulation model are then sent through the analog channel to the amplifier and from there to the motor. The motor is configured in current mode. The motor then creates a torque that is felt by the user as haptic sensations. For further details, please refer to [47].

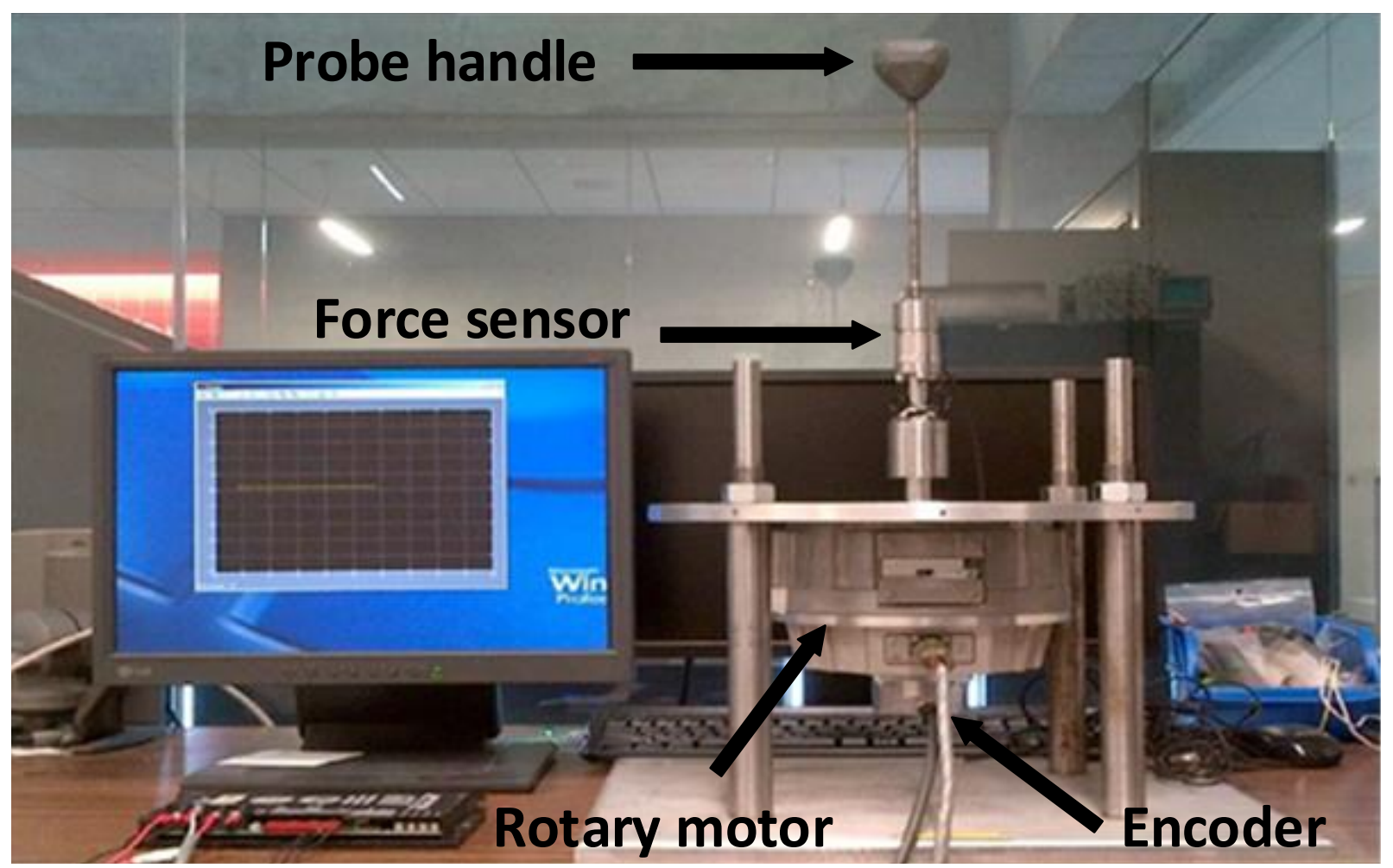

Figure 5.1: First stage of the haptic training simulator [47] 


\subsection{Design Components of the Completed Haptic De- vice}

The current work extends the previous work in [47] by adding a linear stage to the existing rotary stage and coupling the two stages. The following sections will discuss the added components to achieve this goal.

\subsubsection{Hardware Components}

In order to meet the requirements for the simulation of the linear degree of freedom, it is important, first of all, that the actuator has the ability to simulate high forces in the direction of the pedicle axis. The reason that commercial haptic devices are not suitable for our application is that they can typically transmit small forces. For example, the maximum force output offered by the Novint Falcon is about 8 Newtons. Our robot, however, requires a linear actuator that is capable of supplying very large forces (over $100 N)$. This is important for simulating breach effects and dealing with operator's force, since the surgeons typically use extreme forces in this surgical procedure.

The rotary motor weighs approximately $8 \mathrm{~kg}$. Therefore, it would be advantageous that the actuator have a high static friction so as not to move under the weight of the rotary stage. Some other criteria includes supporting a wide range of speeds and having a suitable stroke length.

Pneumatic and hydraulic actuators are also good candidates since they are generally fast and powerful. Electrical motors are, however, more cost effective and more flexible for transportation compared to their pneumatic and hydraulic counterparts since the latter 
requires bulky pumps and valves. Given these requirements, an electrical geared motor was chosen with the load capacity of 100lbs and a speed of approximately 3" per second for the simulation of the linear motion (see Figure 5.2). More specifications of the actuator are given in Appendix A.

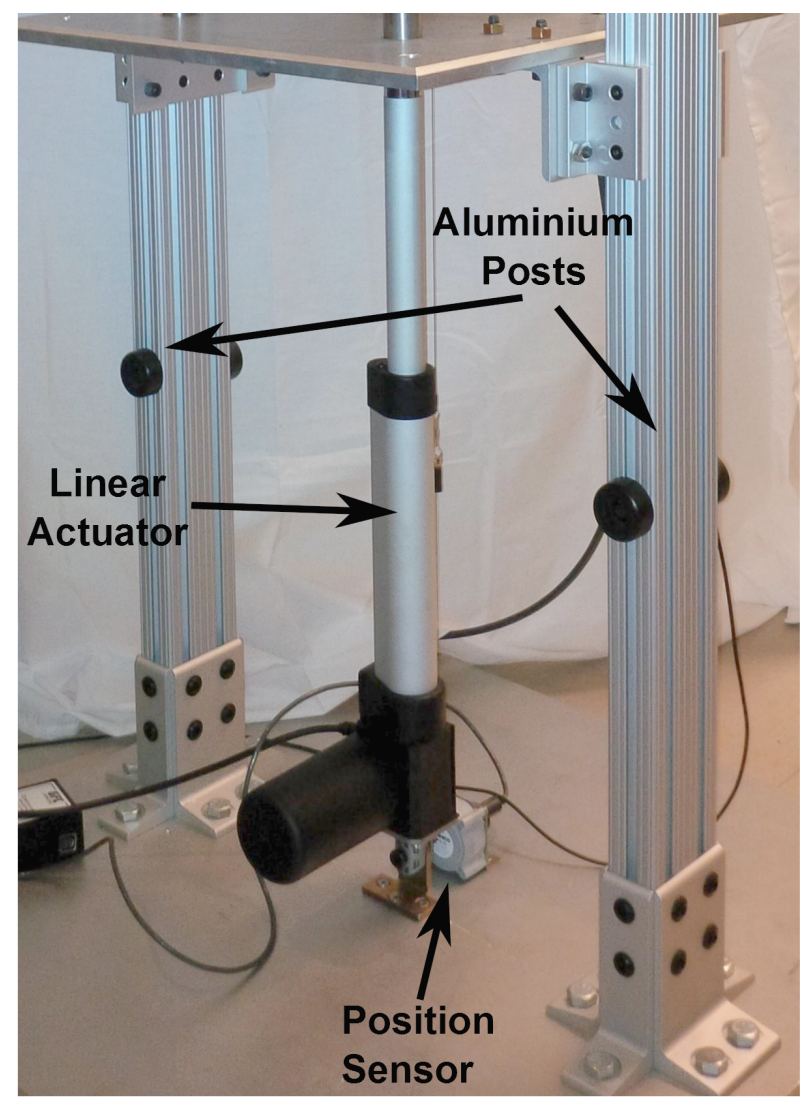

Figure 5.2: Linear stage showing position sensor, linear actuator, and supporting aluminum posts

As shown in the Figure 5.2, two aluminum posts are placed on the sides along the linear actuator to provide vertical support to the platform. The rotary stage of the device is connected to the linear bearings which glide smoothly over the posts. 
For measuring position along the axis of the linear actuator, a position sensor was aligned and coupled to the rotary stage plate as depicted in Figure 5.2. The Celesco SP212 string pot sensor allows for measuring of the position of the top plate with the accuracy of $0.79 \mathrm{~mm}$.

A six-axis transducer is also placed between the probe and the shaft of the motor. This Nano25 F/T sensor enables measurement of the user's force and torque as illustrated in Figure 5.3. The $\mathrm{F} / \mathrm{T}$ sensor provides torque and force information in three directions. The force in the $Z$ direction represents the operator's vertical force and is incorporated into the linear motor controller. The sensor's force resolution in the $Z$ direction is $1 / 16 N$. This high signal-to-noise ratio is a key factor that governs the performance of the theoretical controller on the experimental setup. 


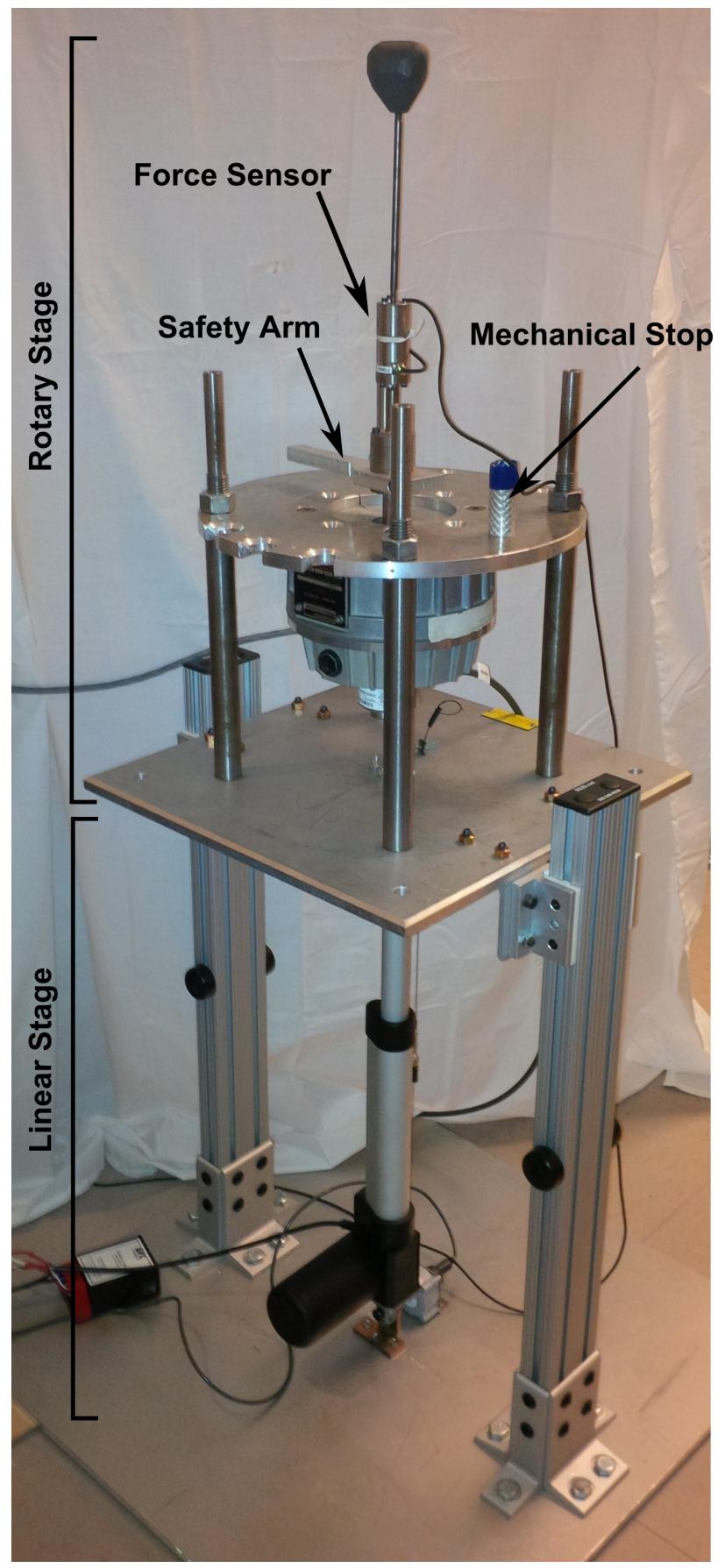

Figure 5.3: Overview of the haptic simulator 
A safety mechanism was also designed and added to the rotary stage as illustrated in Figure 5.3. In case of instability, the rotary motor can rotate forcefully which is dangerous. The safety mechanism consists of a safety arm and a mechanical stop. The former is coupled to the end of the probe and moves with the motor, while the latter is machined on top of the rotary motor. By using such a mechanism, the motor shaft cannot rotate over 360 degrees and the energy in the motor gets dissipated.

Further details regarding the specification of the hardware is given in the Appendix A.

\subsubsection{Hardware/Software Interface}

DAQ hardware interfaces the signals from the sensors to the PC and is responsible for converting analog signals to digital signals or vice versa. Two DAQ cards and one MultiQPCI terminal board were used for communicating signals between hardware and computer.

A motor driver is responsible for controlling the voltage to the linear actuator. It controls the speed and position of the motor. The signal generated in the software is output to the motor driver through the DAQ and terminal board, and then is transmitted to the linear actuator. Figure 5.4 shows connections between various components of the system. 


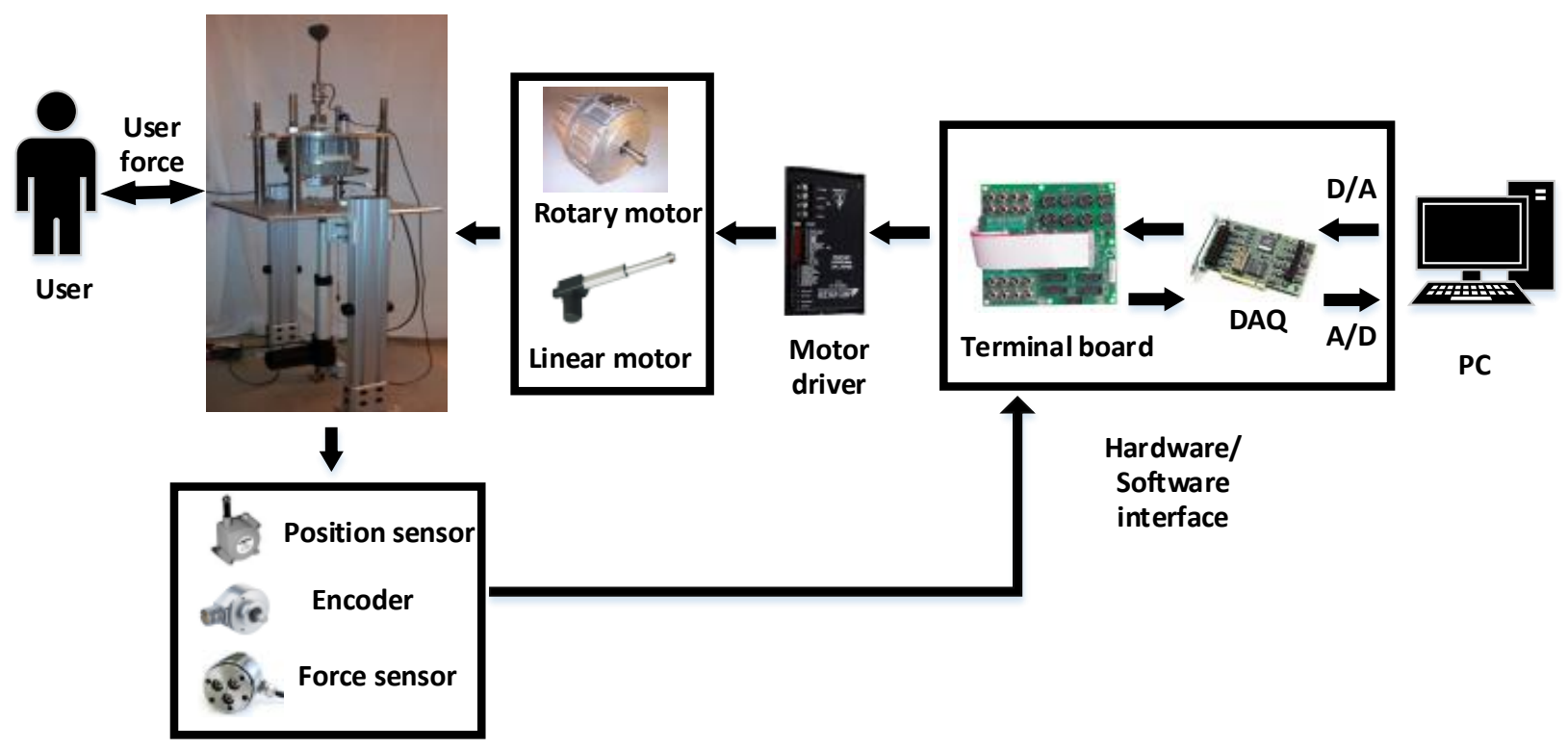

Figure 5.4: System components

\subsubsection{Software and Real-time Component}

For the purpose of real-time simulating effects, control algorithms are executed in hard real-time through Real-time workshop of Matlab/Simulink with a discrete time solver at the sampling rate of $1000 \mathrm{~Hz}$. Acquired data can be displayed, analyzed, and stored on a computer. Matlab also provides built-in graphical tools for developing graphical user interfaces. 


\subsubsection{Design and Implementation of the Proposed Control Tech- niques}

In Section 4.4, potential structures for the simulation of linear haptic effects were proposed. Now that the type of actuator has been determined, the design of the experimental apparatus can be completed.

\subsubsection{Closed loop PID Control Technique: Design and Computation}

As discussed in the previous chapter, for closed-loop control design, we first need to model the dynamic behaviour of the actuator.

Modeling The dynamical behaviour of the linear actuator in the absence of friction can be estimated with a first-order transfer function model:

$$
\hat{G}(s)=\frac{v(s)}{u(s)}=\frac{K_{m}}{J_{m} \cdot s+B_{m}}
$$

where $J_{m}$ represents motor moment of inertia, $B_{m}$ is viscous damping and $K_{m}$ is force constant of the motor and $v(s)$ and $u(s)$ represent velocity and input voltage in the Laplace domain. This model can be simplified by dividing numerator and denominator by $k_{m}$ :

$$
\hat{G}(s)=\frac{v(s)}{u(s)}=\frac{1}{M \cdot s+B}
$$

There are only two parameters to identify. In the existence of friction, the model is extended to: 


$$
v(s)=\frac{1}{M \cdot s+B}(u(s)-d(s))
$$

where $d(s)$ is the input disturbance that corresponds to the effect of friction in the motor.

Parameter Identification Parameter estimation is done in frequency domain using the least square method (LS) [51]. The frequency response function (FRF) of the system can be obtained by substituting s by $j \omega$ in Equation 5.2

$$
\hat{G}(j \omega)=\frac{1}{M(j \omega)+B}
$$

In the experiment, the linear actuator was excited with various frequencies (one frequency at a time) and output responses were stored for further calculation. Each measured data can be written in the form of:

$$
v(t)=F(\omega) \sin (\omega t+\Phi(\omega))+m^{\prime}=\left[\begin{array}{lll}
\sin (\omega t) & \cos (\omega t) & 1
\end{array}\right]\left[\begin{array}{c}
F(\omega) \cos (\Phi(\omega)) \\
F(\omega) \sin (\Phi(\omega)) \\
m^{\prime}
\end{array}\right]
$$

where $F(\omega)$ represents system amplitude, $\Phi(\omega)$ is system phase and $m^{\prime}$ is offset. After extracting the second vector components in the above equation, the phase and magnitude of the response is easily computed and the frequency response is written in terms of real and imaginary components as follows:

$$
G(j \omega)=g(\omega)+j h(\omega), \quad g(\omega)=F(\omega) \cos (\Phi(\omega)), \quad h(\omega)=F(\omega) \sin (\Phi(\omega))
$$


where $G(j \omega)$ represents the measured frequency response of the system. For a good fit to the measured data, according to the model in Equation 5.4, the following equality should hold:

$$
\hat{G}^{-1}(j \omega) \times G(j \omega)=1
$$

In other words,

$$
\frac{1}{M(j \omega)+B} \times(g(\omega)+j h(\omega))=1
$$

Simplifying the above equation for real and imaginary components, we obtain two main equations:

$$
\left\{\begin{array}{l}
B \cdot g(\omega)-M \cdot \omega \cdot h(\omega)=1 \\
M \cdot \omega \cdot g(\omega)+B \cdot h(\omega)=0
\end{array}\right.
$$

Unknown parameters in the above equality are solved using the least square technique which determines the best fit line to the sequence of observed data. Its objective is to minimize the sum of squares of the differences between the observed and computed data. Equation 5.9 can be expressed linearly in terms of unknown parameters in the model, i.e., $M$ and $B$ as in Equation 5.10. Terms without unknown parameters are grouped in the vector $Y$ and the rest of the equation is grouped in two vectors: the vector of unknown parameters $\theta$ and the vector of parameter coefficients $\Phi$.

$$
Y=\Phi \theta
$$

For Equation 5.9, the LS parameters are constructed as: 


$$
Y=\left[\begin{array}{c}
1(N, 1) \\
0(N, 1)
\end{array}\right], \quad \Phi=\left[\begin{array}{cc}
-\omega(1) h(\omega(1)) & g(\omega(1)) \\
\vdots & \vdots \\
-\omega(N) h(\omega(N)) & g(\omega(N)) \\
-\omega(1) g(\omega(1)) & h(\omega(1)) \\
\vdots & \vdots \\
-\omega(N) g(\omega(N)) & h(\omega(N))
\end{array}\right], \quad \theta=\left[\begin{array}{c}
M \\
B
\end{array}\right]
$$

where $N$ is the number of testing frequency. $1(N, 1)$ and $0(N, 1)$ are vectors of size $N \times 1$ filled with scalar one and zero, respectively. $g(\omega)$ and $h(\omega)$ are the real and imaginary component of the measured response, and $\omega$ is the testing frequency. A total of 14 tests were conducted by applying sine waves with frequencies in the range of 1 to $120 \mathrm{rad} / \mathrm{s}$. M and $\mathrm{B}$ were estimated to 0.0217 and 0.2177 , respectively. The frequency response of the identified model as well as the actual output response are illustrated in Figure 5.5. 

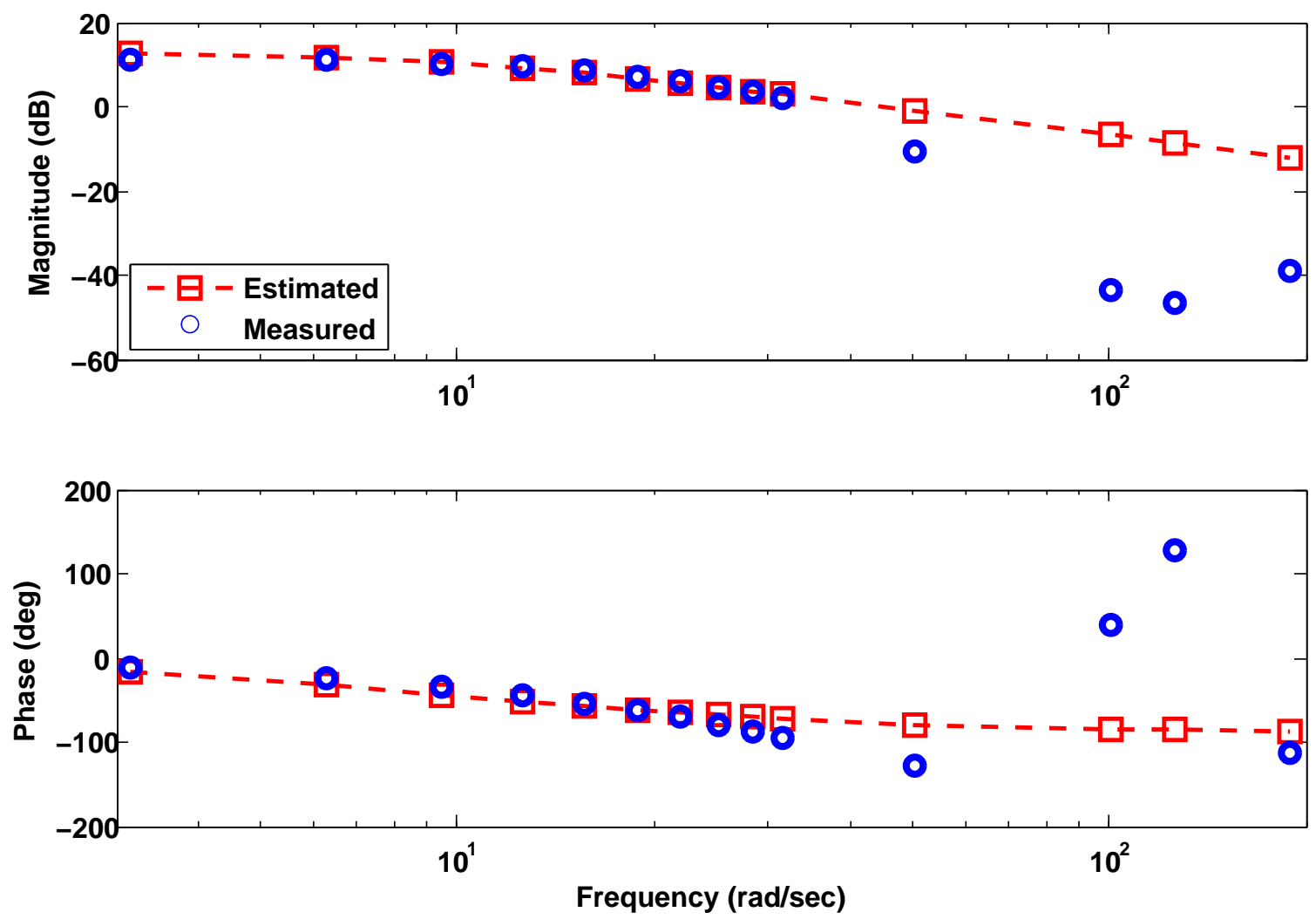

Figure 5.5: Frequency response of the identified model vs. the actual output response

It can be seen in the low frequencies that the identified model represents the actual response. However, at high frequencies, responses deviate considerably. This means that the model is not capable of representing the actual system at those frequencies. This mismatch is later improved by looking at the time domain response and modifying the coefficients. Frequency measurement provides a good starting point, time domain verification, however is more relevant to haptics as it is easier to describe and modify effects in the time domain.

In the time domain test, a series of square waves with random amplitude is generated as shown in the second subplot in Figure 5.6. This signal is fed to the actual motor as 
well as the identified model. The velocity of the actuator is then calculated by taking the derivative of the position sensor data and is shown with the green color in the first subplot. The red color represents the velocity of the identified model. Velocity tracking is more critical in this study because it is of importance to drive the motor quickly. Measured data is very noisy. This is due to the presence of noise in the position sensor data and the fact that by taking the derivative the noise is amplified. However, it is apparent that the trend captured from the estimated model can represent the actual model.
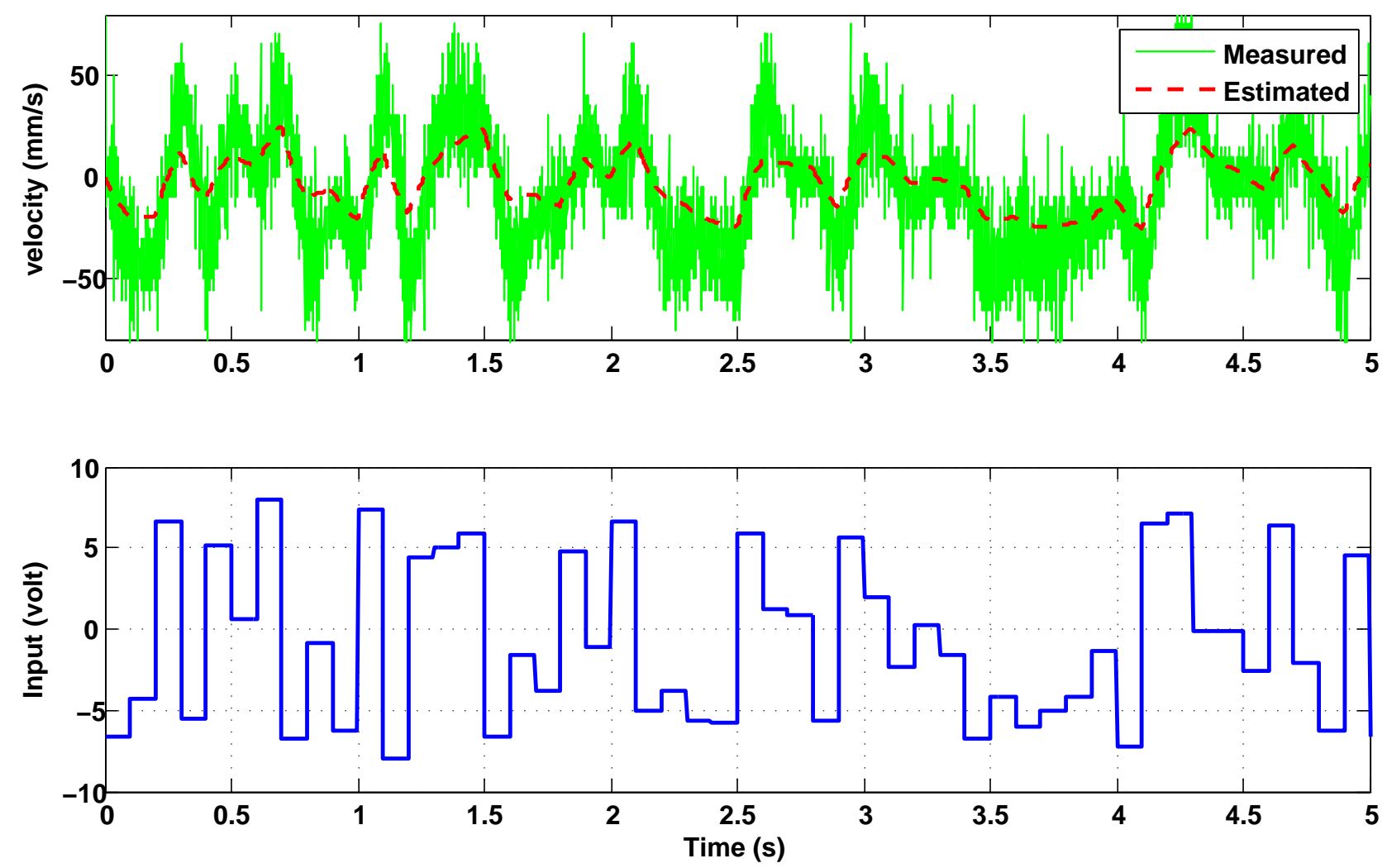

Figure 5.6: Velocity response estimation of identified model vs actual measurements

Minor adjustments to the estimated $\mathrm{M}$ and $\mathrm{B}$ are made by trial and error to improve the 
theoretical model with respect to the measured velocity especially in higher frequencies. In order to do this, Equation 5.2 is first written in the following format

$$
\hat{G}(s)=\frac{\frac{1}{M}}{s+\frac{B}{M}}=\frac{a}{s+\tau}
$$

The velocity response of a range of $a$ and $\tau$ are tested and overlaid in one graph as shown in Figure 5.7. The mean squared error between these signal and measured output is calculated and presented in Table 5.1. The smallest value belongs to the response shown by the black color $(a=200$ and $\tau=35)$. This is associated with $M$ and $B$ of 0.005 and 0.175, respectively. Although the adjusted model does not precisely capture the trends, it provides reasonably a better fit to the rise and fall in the measured signal.

Table 5.1: The mean squared error between the measured signal and output of adjusted models

\begin{tabular}{|c|c|}
\hline Signal & Mean Squared Error \\
\hline \hline Estimated & 544.74 \\
$\mathrm{a}=200, \tau=35$ & 476.68 \\
$\mathrm{a}=200, \tau=40$ & 500.38 \\
$\mathrm{a}=250, \tau=35$ & 549.42 \\
$\mathrm{a}=250, \tau=40$ & 544.74 \\
\hline
\end{tabular}




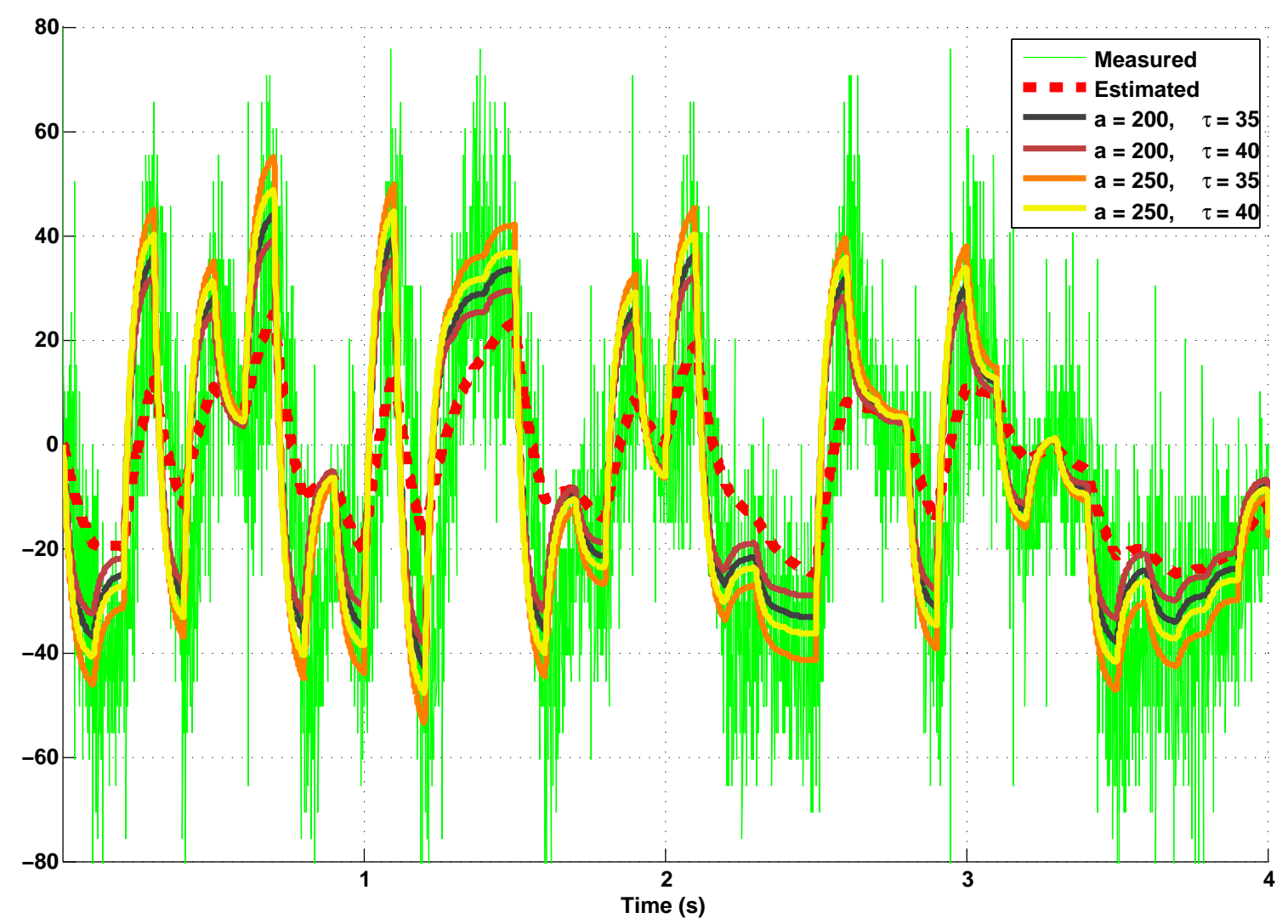

Figure 5.7: Making minor adjustment to the parameters $a$ and $\tau$. The black line shows a better fit than the red line (estimated velocity).

Friction Motors have internal friction and therefore display nonlinear behavior. Static friction (also called stiction) causes the motor to remain stationary even when voltage is applied to it. As the motor starts moving, the friction decreases and it turns to dynamic friction. In order to treat the motor as a linear model, the friction needs to be compensated.

A simple technique to determine static friction is to increase the voltage input and look for the smallest value that makes the motor move. Six tests were run for measuring friction in the downward direction. Test results are shown in Table 5.2. 
Table 5.2: Experimental result for measuring static friction

\begin{tabular}{|c|c|c|}
\hline Test No. & Stiction (downward direction) & Stiction (upward direction) \\
\hline \hline 1 & -1.43 & 2.25 \\
2 & -1.20 & 2.28 \\
3 & -1.43 & 2.17 \\
4 & -1.45 & 2.44 \\
5 & -1.37 & 2.16 \\
6 & -1.31 & 2.43 \\
\hline
\end{tabular}

As observed, static friction is not symmetric for upward and downward movement due to the significant load of the top plate. The mean value and standard deviation of the observed data are -1.37 and 0.10 for the downward stiction and 2.29 and 0.12 for the upward stiction. To compensate the kinetic friction, values smaller than the experimentally estimated values of static friction are used in the closed loop control, so that instability due to friction compensation does not occur.

Control Objectives Now that the linear model of the system as well as the friction in the actuator is identified, we can design the PID controller. As discussed earlier, the goal is to perform precise position control with small overshoot and quick response. As for the linear progression simulation, the reference input to this closed loop structure is composed of series of steps that are generated from the user force (see Section 4.4.1.1). Breach simulation also uses a similar structure for the step with the size of desired displacement. Therefore, the designed controller should achieve desirable performance for a wide range of step sizes. 
For step sizes of $2 \mathrm{~mm}, 5 \mathrm{~mm}, 10 \mathrm{~mm}, 20 \mathrm{~mm}$, and $40 \mathrm{~mm}$, the PID controller parameters were designed. Initially, PID coefficient were designed using loop shaping control techniques, for an open loop phase margin of 50 degrees at the cross over frequency of 50 $\mathrm{rad} / \mathrm{s}$. Then, these values were tested on the actuator. The parameters were then adjusted by trial-and-error for each case. The proportional and integrator coefficients were considerably lowered to avoid saturation. By varying the proportional and derivative coefficient, a reasonable overshoot is obtained. By increasing the integral coefficient, the steady state error is improved, while at the same time the overshoot was worse and the transient response was slow. A trade-off in tuning the control parameters is required in achieving an adequate overall performance. Overall, the modified controllers had lower gains in comparison with the initial controller and had a better phase margin (see Figure 5.8).

In the actual implementation, due to the presence of wind-up effects, the integrator component of the controller was substituted by a moving window integrator that only use the last 500 samples for cumulative summation. This modification improved the performance with much less control effort. As for the derivative portion, the signal was low-pass filtered in order to achieve a clean signal. These controllers were then used on the system with an input composed of a series of steps with various step sizes and the results are illustrated in Figure 5.9 and 5.10. 


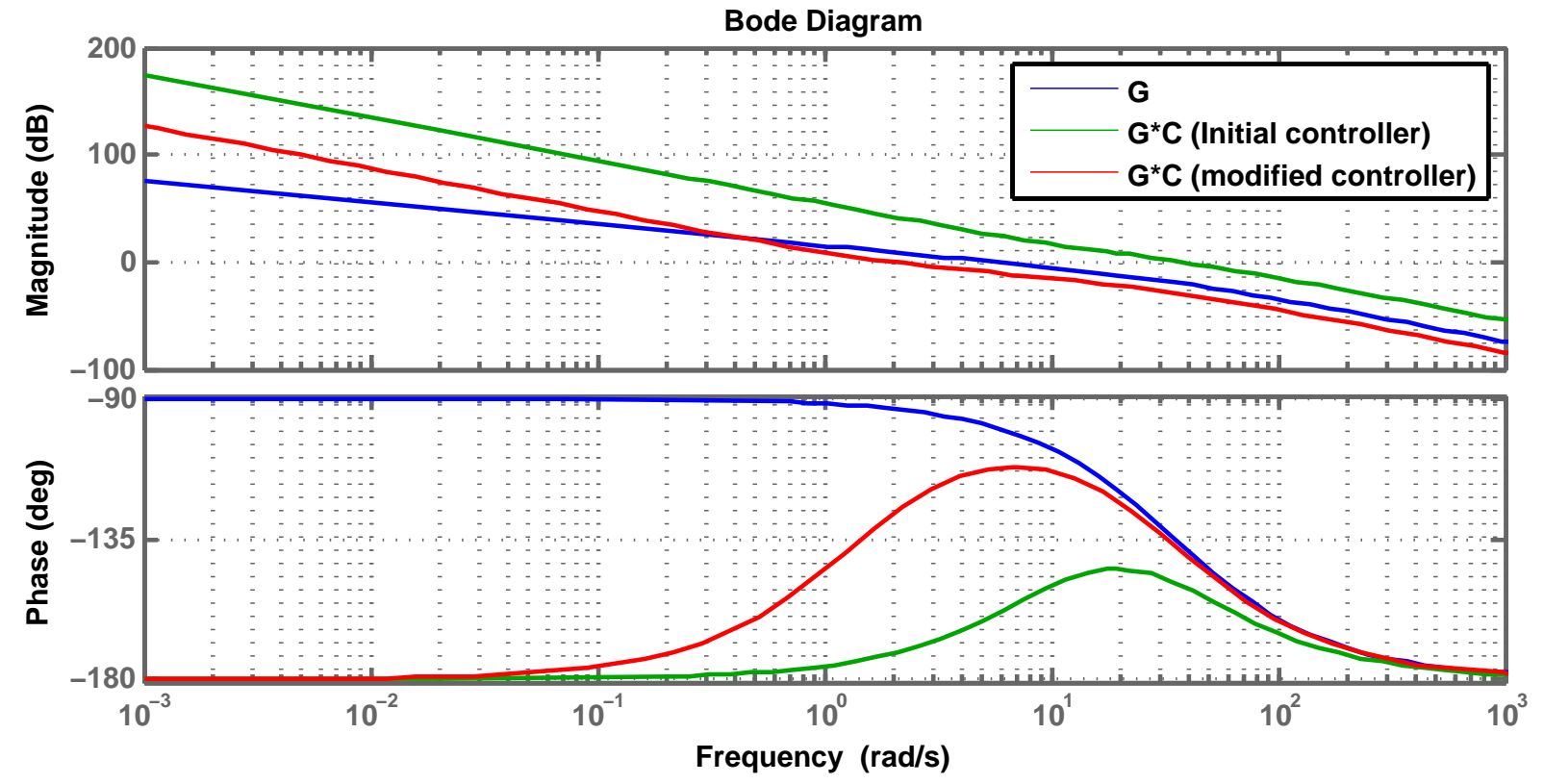

Figure 5.8: Comparing open loop bode plots; first one is without the controller, second one is with the initial controller and last one is with modified controller (adjusted for step size of 10mm) 


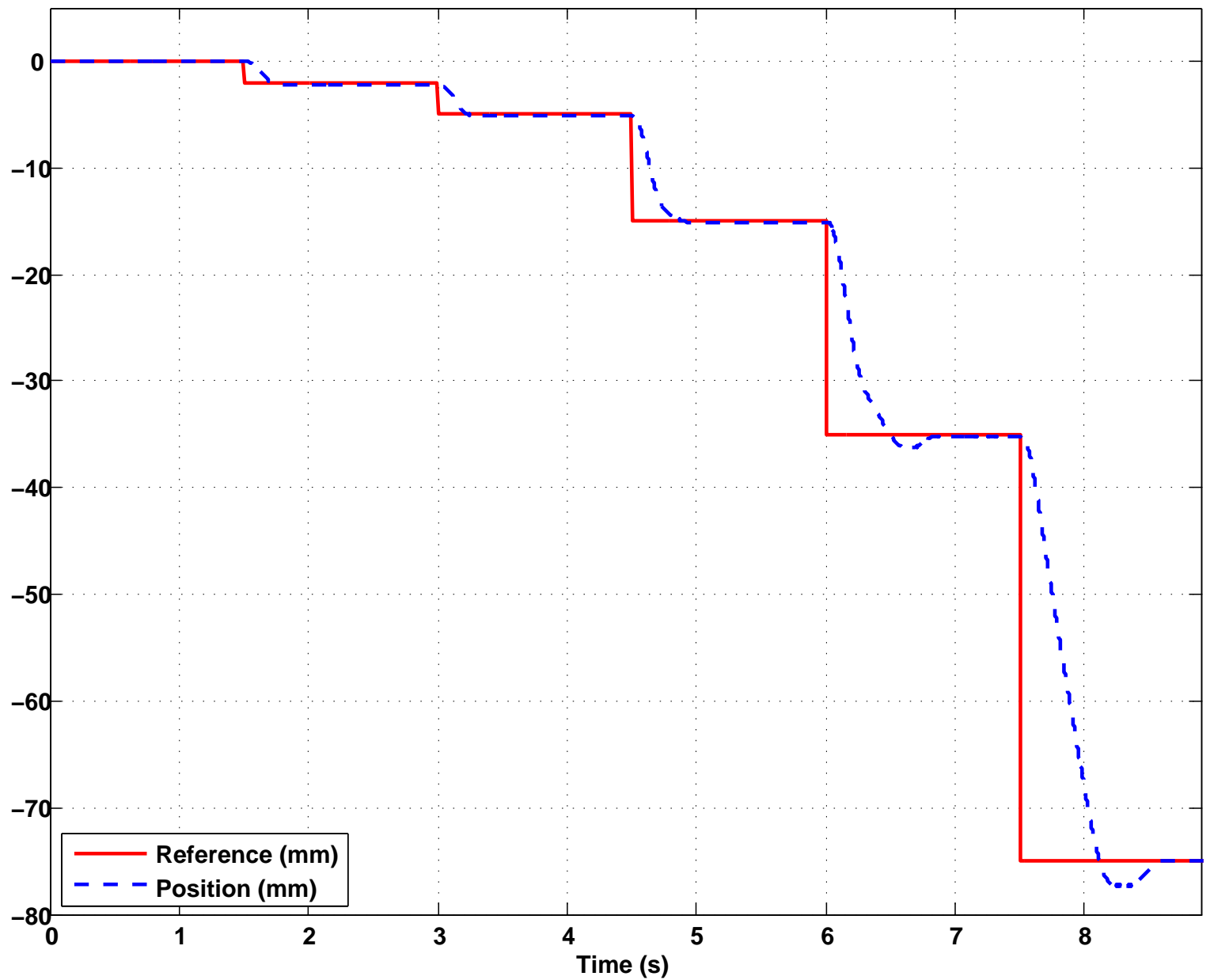

Figure 5.9: Output response of the system with the controller tuned for $5 \mathrm{~mm}$ step size on a set of steps as a reference 


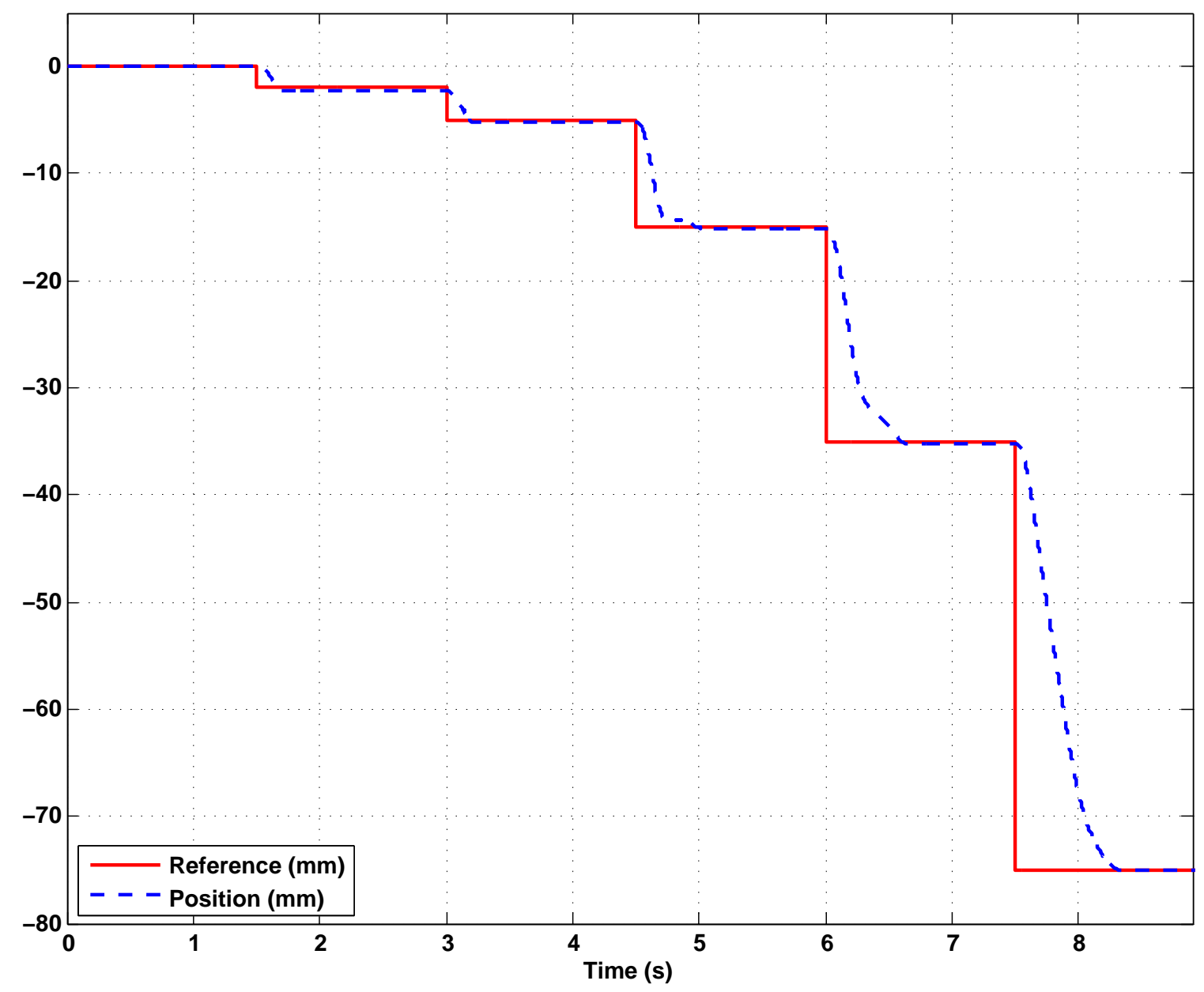

Figure 5.10: Output response of the system with the controller tuned for $40 \mathrm{~mm}$ step size on a set of steps as a reference

In the Figure 5.9, the controller that was tuned for the $5 \mathrm{~mm}$ step size is employed. As seen, controller is not able to quickly respond to the large step size. Moreover, it causes considerable overshoot for larger step size.

On the other hand, Figure 5.10 illustrates the result for the experiment with the con- 
troller designed for $40 \mathrm{~mm}$ step size. Due to the saturation in the control signal for smaller changes in the reference, an unexpected pattern appears in the tracking. It is also worth noting that even for the $40 \mathrm{~mm}$ step size, the transient response is not fast enough. This, in fact, is attributed to the hardware limitation.

Therefore, no unique controller parameters meet the design requirement for various step sizes. Due to the existence of nonlinearity in the system such as coulomb friction and saturation, this objective is very difficult to maintain and as step size varies, various issues such as overshoot, steady state error, and lagging are noticeably observed.

At this point, we need to use expert surgeons' feedback in the development cycle to see if the design performance is adequate. These studies are investigated in the next chapters.

\subsubsection{Open Loop Control: Design and Computation}

As discussed in Section 4.4.2, for the open loop control approach, linear motion incorporates three parameters to produce different haptic effects and the parameters are tuned with clinical testing. As for the breach simulation, it is crucial to use the maximum speed of the actuator. As discussed in the previous chapter, for the proposed approach, a mapping relation is required between the applied pulse widths and the displacement achieved. To find the relation, in a series of tests, the actuator is fed with pulses of maximum voltage and various pulse width. Figure 5.11 shows the test results. The displacement of the actuator is increased as the voltage is applied over a longer period of time. The gain ratio which maps pulse duration and achieved displacement is then calculated for each test result. Over the 6 measurements, mean value and standard deviation of the results are 7.44 and 0.17 respectively. The average value is used as the mapping gain and the relation between desired displacement and required pulse duration is calculated as: 


$$
\text { PulseDuration }=\frac{\text { Displacement }}{\text { MappingGain }}, \quad \text { MappingGain }=7.44
$$
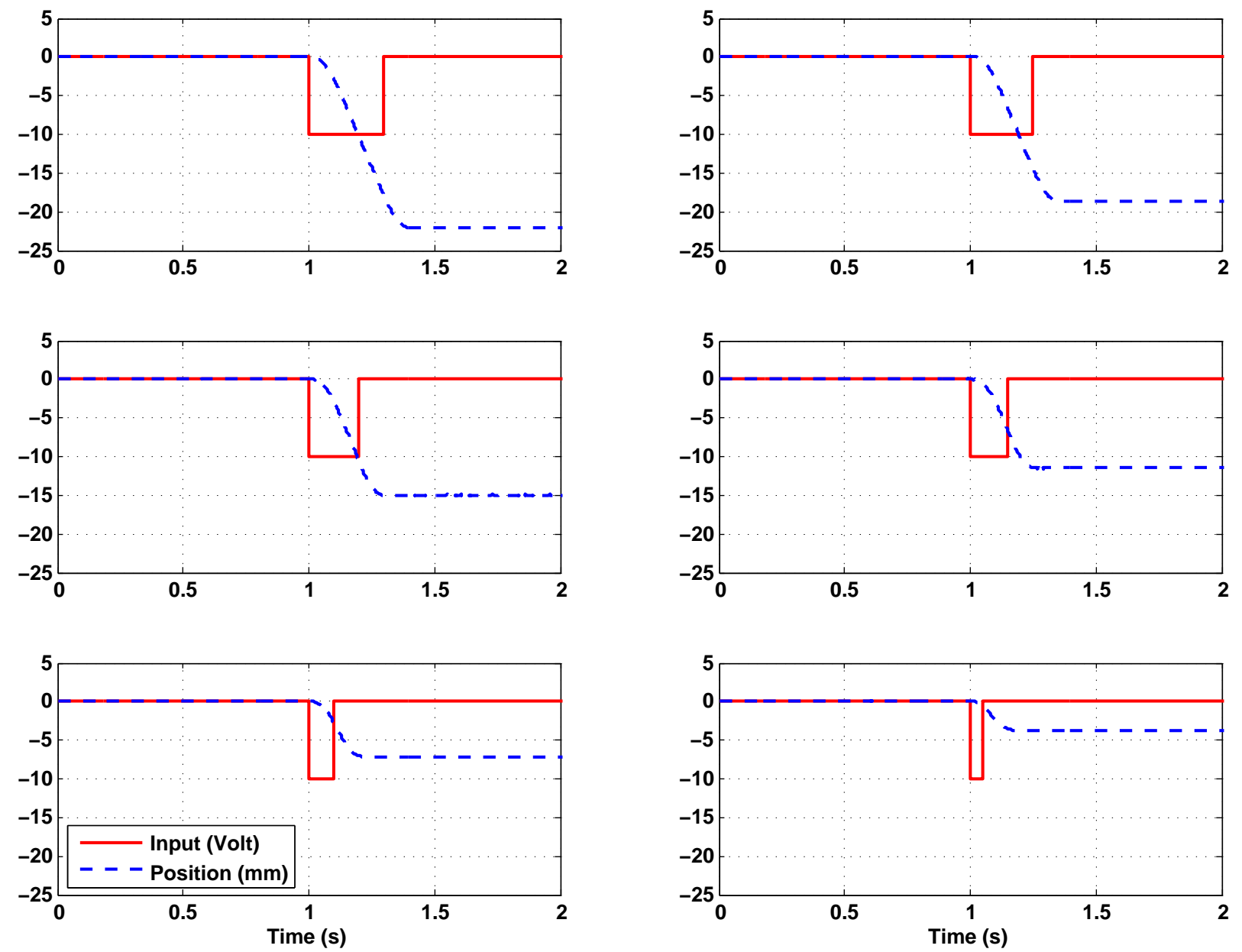

Figure 5.11: Displacement of the actuator with maximum input voltage and six different pulse duration including $0.3,0.25,0.2,0.15,0.1$ and 0.05 second. 


\subsection{Summary of Haptic Model Parameters}

To summarize, the haptic model for probe channeling pedicle screw insertion has seven parameters for linear progression simulation and two for breach simulation. The parameters are further clinically tunned (see Chapter 6) such that the effects create realistic feeling.

\section{Rotary Stage:}

Detent interval Determines the frequency of vibration.

Detent width Determines the detent sharpness by changing the detent width

Detent magnitude Determines the vibration magnitude.

Viscous friction coefficient determines the magnitude of viscous friction.

\section{Linear Stage, linear progression:}

Frequency Determines frequency of vibration in linear progression. For a small value, there would be less number of steps in a linear progression. In other words, there is less vibration in linear movement.

Duty cycle Determines the smoothness of linear progression. By increasing this value, each motion step can go further with the same amount of force applied on it. If it is at its maximum value (smoothest), then one feels continuous motion without any pause. 
Scaling gain Determines the speed of motion. This parameter can specify amount of pressure that should be applied to get movement. Maximum input voltage of the actuator is 12 volts. Given this limitation, after a certain amount, the effect remains the same.

\section{Linear Stage, breach:}

Force threshold Determine the force at which the breach event occurs.

Desired displacement Determines how far probe should fall downward when a breach event occurs.

\subsection{Summary}

In this chapter, the components of the developed experimental apparatus were described. First, a brief explanation of the rotary stage platform was provided. Selection of the hardware and software components for the linear stage with regards to the design requirements are then discussed. Subsequently, proposed control techniques for the linear stages are applied on the developed experimental apparatus. The chapter concludes with the summary of the haptic model parameters. The next chapter will present the testing protocol that is employed for the clinical tuning of the haptic model parameters. 


\section{Chapter 6}

\section{Clinical Testing}

\subsection{Objectives}

In this chapter, the detailed protocol of how the haptic model parameters are tuned will be presented. In Leung's thesis [47], it was verified that the rotary stage is capable of simulating realistic haptic sensations as the probe is rotated inside the pedicle. In the current work, we study the fidelity of simulation for the second DOF which is the translational motion. We also implement and study the coupling between the two stages. The haptic model incorporates parameters that can be adjusted to create different haptic sensations to the user. As a result, when the user applies the torque and force on the probe, the proper force and torque will be computed and reflected to the user. Clinical testings are performed to tune the haptic model parameters such that effects replicate the

probe channeling through the pedicle. Tuning is performed for both the rotary stage and the linear stage. Tests are carried out by orthopedic surgeons and neurosurgeons who have experience in pedicle screw insertion surgery. 


\subsection{Ethics Statement}

The protocol of this research was approved by the office of research ethics at University of Toronto. Each participant was provided a consent letter prior to beginning the experiment. All relevant ethics documents are included in Appendix B.

\subsection{Research Questions}

The research questions that are investigated are as follows:

Q1: Can a reference set of values be found that replicate the surgeon's interpretation of the surgical scenario?

Q2: How are the rotary stage parameters influenced in the presence of linear effects?

Q3: Do the results differ across different expertise levels?

Q4: Can the simulator serve as a useful tool in the education of surgical trainees for teaching channel creation in pedicle screw insertion?

\subsection{Method}

The haptic simulation model has been designed and developed in an effort to simulate the biomechanical characteristics of the surgical procedure. Haptic model parameters are tuned with participating surgeons such that the haptic sensation imitates the texture of the real pedicle bone as a probe is passed through. In Leung's thesis [47], the tuning 
experiments for the rotary stage were done for three anatomical scenarios. The focus in this test, however, is a healthy bone with normal density. For example, a low-density osteoporotic bone in an elderly patient would be softer than our focus. Similarly, a highdensity bone in a young and active scoliosis patient would be harder than the bone in this study.

The test is designed with the focus only on haptic sensations. Therefore, no complementary graphical interface is presented to the user. To make the interaction with robot more similar to the actual surgery, the robot is covered by a fabric cloth and only the probe is exposed.

Although the rotary stage parameters were previously tuned, they are re-adjusted in this experiment along with the linear stage parameters for the following reasons. First, a few modifications had been made to the implementation. As well, adding the linear DOF may affect the participants' perception of the haptic parameters in the rotary stage.

During the experiment, the researcher changed parameter values as requested by the surgeon. Results from prior tests with an expert surgeon were useful in picking the initial values presented to the participant. To reduce the error of expectation in the test, tuning in each scenario was initialized with an approximate value $50 \%$ below and above the preobtained value. Each session takes on average from 25 to 35 minutes. More details will be presented later. 


\subsection{Participants}

There were 11 surgeons ${ }^{1}$ composed of 8 orthopedic surgeons and 3 neurosurgeons enrolled in the study. Among the orthopedic surgeons, one was senior, three were fellows, and four were residents. Neurosurgeons consisted of one resident, one fellow, and one senior. The senior surgeons each had 15+ surgical experience. The fellow surgeons had 6-10 years of surgical experience and medical residents' experience varied between 4-9 years.

\subsection{Protocol Outline}

All data collection was carried out at the University of Toronto. Tests are completed in one session for each participant. Each session is composed of the following steps:

- Didactic session:

At the beginning of the session, a brief description of the haptic device is given. It is demonstrated how the computer generates the haptic sensations for both stages. The purpose of the experiment and a quick overview of the test is also provided.

- Set-up:

Each participant stands beside the device. He is positioned such that he cannot see the computer display. A three-level step stool is provided in order for the participant to stand at a comfortable and natural height near the device. The participant then holds the probe with one hand and uses the top plate as a support for the other hand

\footnotetext{
${ }^{1}$ Two were female and nine were male.
} 
if needed. The researcher on the workstation side is able to use the graphical interface to tune the parameters based on the surgeon's verbal instructions (see Figure 6.1).

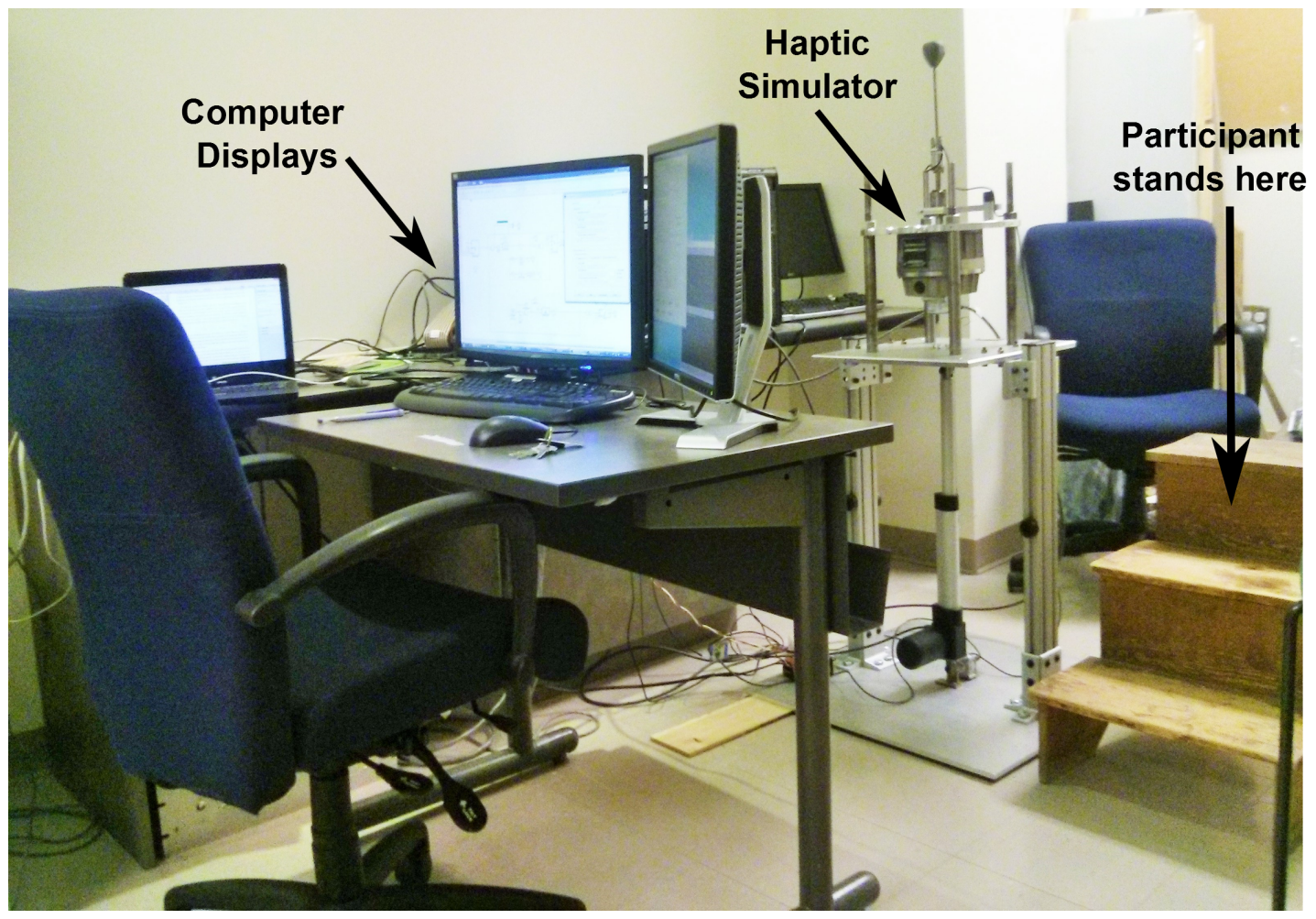

Figure 6.1: Workstation

- Training session:

Prior to the test session, a training session is held. The haptic model and the effects are introduced to the surgeons. First, the main haptic effects such as the viscous damping effect, the pure vibration effect and the pure linear progression are presented to the participant. Following that, the parameters associated with each effect are introduced. Each model parameter is changed over a wide range of values, while other parameters remain fixed. This allows the surgeon to experience the haptic 
sensations related to each model parameter. After talking with surgeons, different terminologies were used to describe some of the parameters to the surgeons so to make them self-explanatory (See Table 6.1).

Table 6.1: Terminology used in testing

\begin{tabular}{|l|c|c|}
\hline & Parameter Name & Terminology Used in Testing \\
\hline \hline Rotary stage & Detent interval & Frequency of vibration \\
& Detent width & Detent (bump) width \\
& Detent magnitude & Magnitude of vibration \\
& Viscous friction coefficient & Viscous friction coefficient \\
\hline \hline Linear Stage & Frequency & Frequency of vibration \\
& Scaling gain & Resistance coefficient \\
& Duty cycle & Smoothness coefficient \\
\hline
\end{tabular}

- Clinical Tuning:

After the preliminary training, surgeons are asked to tune the parameters so that the simulation is equivalent in feel to that of a normal, healthy bone as opposed to an older, osteoporotic bone or a cadaver bone.

The model parameters are set to the data collected from an expert surgeon. Then, the testing is started. While adjusting the first parameter, all other parameters are set to values from the expert's level. The target parameter is tuned twice; once initialized with a random value above the expert's level and once below the expert's level. The average of the two results is then calculated and set to the device. Following that, the same procedure is repeated for tuning the next parameter, with the only exception 
that the previously tuned parameter is kept at the tuned level while others are still at the expert's level. The order of parameter tuning was from the biggest effect to the smallest based on expert surgeons' assessments.

Once all parameters are adjusted and set to the device, surgeons are given another chance to feel their tuning and perform any final tuning that they may think is needed. Tuning all parameters at the same time could be confusing and misleading, but tuning each parameter separately could also cause a response bias. This step of final tuning is aimed to reduce the participant's response error.

- Recording Signals:

All of the tuned values are set to the simulator parameters and then the surgeon is asked to perform the procedure of probe channeling on the simulator as he would perform it in a live human surgery. The surgeon's force and torque profile are recorded, as well as the linear and angular positions of the probe.

- Survey Questionnaire:

At the end of each trial, the surgeons' feedback was solicited on four questions. Responses to the questions were rated on the five-point Likert scale. These questions are as follows:

1. The haptic sensations associated with the rotation of the probe was simulated realistically.

\begin{tabular}{|c|c|c|c|c|}
\hline Strongly Disagree & Disagree & Undecided & Agree & Strongly Agree \\
1 & 2 & 3 & 4 & 5 \\
\hline
\end{tabular}


2. The haptic sensations associated with the linear progression of the probe was simulated realistically.

\begin{tabular}{|c|c|c|c|c|}
\hline Strongly Disagree & Disagree & Undecided & Agree & Strongly Agree \\
1 & 2 & 3 & 4 & 5 \\
\hline
\end{tabular}

3. Overall, the simulator produced realistic haptic sensations felt during probe channeling.

\begin{tabular}{|c|c|c|c|c|}
\hline Strongly Disagree & Disagree & Undecided & Agree & Strongly Agree \\
1 & 2 & 3 & 4 & 5 \\
\hline
\end{tabular}

4. The simulator could potentially be a useful tool for teaching pedicle screw insertion surgery.

\begin{tabular}{|c|c|c|c|c|}
\hline Strongly Disagree & Disagree & Undecided & Agree & Strongly Agree \\
1 & 2 & 3 & 4 & 5 \\
\hline
\end{tabular}

\subsection{Summary}

The detailed testing protocol for tuning the model was presented in this chapter. The method of testing, background of the surgeon participants, research questions and protocol of each testing trial were throughly explained. The next chapter will cover the discussion on the research questions and statistical analysis of the collected data. 


\section{Chapter 7}

\section{Discussion}

The results of the clinical testing and the analysis will be presented and discussed in this chapter. The clinical testing follows the protocol explained in Chapter 6

\subsection{Pilot Studies Prior to Official Testing}

Prior to the official clinical testing, the effectiveness of the designed controllers for the simulation of haptic effects was investigated in a pilot study with two orthopedic surgeons. One of them is a fellow surgeon with 10 years of experience. The other is an experienced pediatric spinal deformity surgeon with more than 25 years of experience from an acute care hospital who performs approximately one-third of all spine surgeries in Canada. It is worth mentioning that there are relatively few complex spine surgeons in Canada. The mentioned expert is the one who supplied the starting values in Section 6.6.

The haptic effects that were generated using closed loop PID techniques were not able to simulate the actual effects felt by these experts in real surgery. Several sets of controller 
parameters were used in these tests both to simulate linear progression and breach effects. The surgeons reported that the effects are not realistic enough when they insert high forces or apply forces very quickly. These procedures are crucial in some anatomical scenarios such as breach or hard bone. Generally, with the controllers designed for small step sizes, there was slow reaction and occasional overshoot for higher step sizes, and with the controllers designed for higher step sizes, saturation appeared in actuator displacement. For a linear system with the step input of any size, the response remains the same and it only scales. Our actuator is, however, prone to nonlinearities such as friction and saturation, and creates the mentioned issues in the control loop.

Due to the fact that the closed loop bandwidth was not sufficiently high, the controller could not create the fast drops required to simulate the actual surgical procedure without causing saturation or considerable overshoot. In other words, it would mean the probe jumps up suddenly. It should be noted that an overshoot is never felt in a real operation and should be avoided in the haptic simulator as well. This technique might be suitable for a faster actuator which has less friction, but it does not provide sufficient accuracy for the current experimental apparatus. Since this technique was not satisfactory, it was dismissed at this point.

One could feed back the acutator's acceleration in the loop, however, it has insignificant effects on the measured force. The maximum speed of the linear actuator is about $3 \mathrm{in} / \mathrm{sec}$ and it is loaded by around $10 \mathrm{~kg}$ mass (approximate mass of rotary motor and aluminum plate). Assuming the actuator accelerates at a constant rate from zero to 0 to $3 \mathrm{inc} / \mathrm{sec}$ in one second, its acceleration is $3 \mathrm{in} / \mathrm{s}^{2}$. According to the D'Alembert's principle, actuator can create the inertial force of approximately 0.75 Newton which is negligible compared to the forces that surgeons apply (which is around 100 Newtons) and it does not impact the measured force very much. Since the effective inertial force is insignificant, it would be 
legitimate to use force in open loop without feeding back the acceleration.

The open loop technique, on the other hand, was able to generate the subtle torque and force effects that closely match the actual sensations felt in the surgery during linear progression.

However, breach simulations using the same technique did not successfully resemble the actual haptic sensations during surgery. The fact that the linear actuator does not have a sufficiently high bandwidth prevents it from creating the quick drops which are crucial in the breach event. Unlike the closed loop PID technique, the open loop approach never causes an overshoot in the response, but achieving a fast transient response is still problematic due to the hardware limitations. It is hoped that in future research, we can employ faster linear actuators in the design. Before human testing, the speed of the actuator was thought to be sufficiently fast based on observation of real surgery. At this point, we dismiss clinical tuning for the breach simulation in this study.

\subsection{Discussion on the Results from Official Testing}

During the clinical testing, rotary stage parameters (detent interval, detent width, detent magnitude and viscous friction coefficient) and linear progression parameters (frequency, duty cycle, scaling gain) were tuned. For the simulation of the linear progression haptic model, open loop techniques were employed. In the following sections, each of the research questions are investigated separately. 


\subsubsection{Discussion of Research Question No. 1}

Can a reference set of values be found that replicate the surgeon's interpretation of the surgical scenario?

Confidence interval (CI) is used to estimate the benchmark reference set of values for haptic model parameters. In statistics, 95\% CI provides a range of population values with which a sample statistic is consistent at a 95 percent level of confidence. In other words, any other surgeon would come up with a value that lies within that range with this particular confidence interval. Before computing CI, outliers are removed from the data set. An outlier is an extremely high or low value in a set of data. Interquartile range (IQR) is used to find outliers. The range between 25 th and 75 th percentile values is called interquartile range. Outliers are defined as values that fall more than 1.5 IQR's above the 75th percentile or 1.5 IQR's below the 25th percentile [52].

There were four potential outliers in the data. Among the rotary stage parameters, detent interval and detent width had one outlier. As well, among the linear stage parameters, frequency and duty cycle contained one outlier each. All outlier data were from the two neurosurgeons. Outliers in the rotary stage parameters were associated with a fellow neurosurgeon and outliers in the linear stage parameters with a resident neurosurgeon. This procedure is less commonly performed by the neurosurgeons. Besides, these participants conducted a surgical workshop on cadavers within the three days of the clinical testing and that could potentially affect their perception of operating on a healthy bone. To maintain consistency, all rotary stage parameters from this fellow neurosurgeon are removed, as well as all linear stage data from the aforementioned resident neurosurgeon.

Box plots are used to show the distribution of the collected data. In Figure 7.1, maximum and minimum data are shown. The ends of box is $25 \%$ and $75 \%$ of the distribution 
and the median value is the horizontal red line inside the box. Green lines indicate boundaries for $95 \%$ confidence interval. Since the sample size is small, t-distribution is used for finding confidence interval. Assuming that the population distribution is normal and the sample size is in t-distribution, $\mathrm{t}$ value of 2.262 is employed from the $\mathrm{t}$-table for the sample size of 10 and a two-tailed 5 percent critical value [53]. The t-value is then used in calculating upper and lower boundaries. Table 7.1 reports median, mean, standard deviation and boundaries of confidence interval.

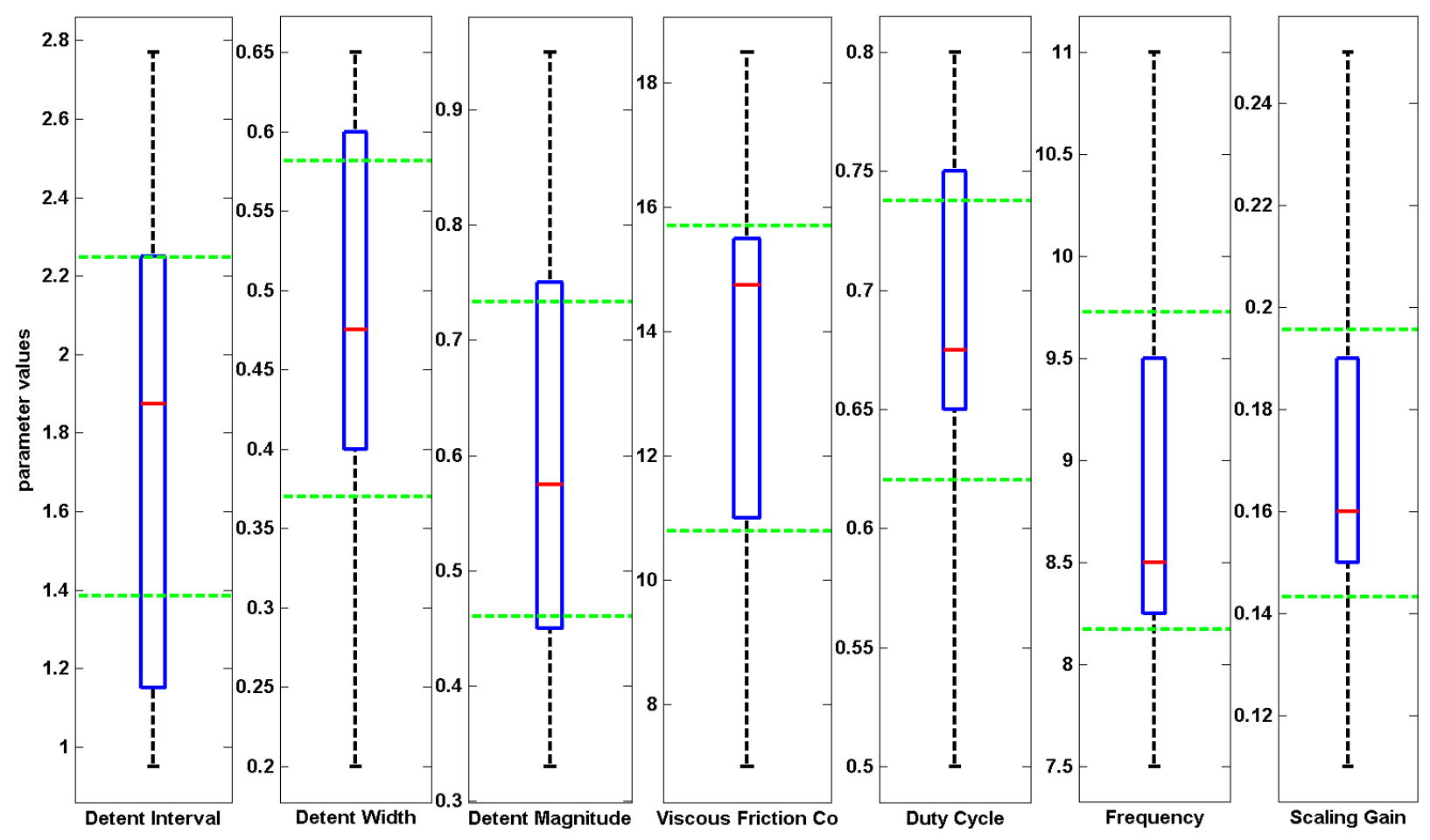

Figure 7.1: Distribution of parameter values obtained from clinical testing with experienced surgeons. Outliers are excluded from the data set (therefore $\mathrm{N}=10$ ). The boundaries of confidence interval for each parameter is indicated by green horizontal lines. 
Table 7.1: Summary statistics

\begin{tabular}{|c|c|c|c|c|c|c|}
\hline & \multirow[b]{2}{*}{ Parameter } & \multicolumn{2}{|c|}{ 95\% Confidence Interval } & \multirow[b]{2}{*}{ Median } & \multirow[b]{2}{*}{ Mean } & \multirow[b]{2}{*}{ Std. Deviation } \\
\hline & & Lower Boundary & Upper boundary & & & \\
\hline \multirow[t]{4}{*}{ Rotary stage } & Detent interval & 1.36 & 2.27 & 1.87 & 1.81 & 0.63 \\
\hline & Detent width & 0.36 & 0.58 & 0.47 & 0.47 & 0.15 \\
\hline & Detent magnitude & 0.45 & 0.74 & 0.57 & 0.59 & 0.20 \\
\hline & Viscous friction coeff. & 10.66 & 15.83 & 14.75 & 13.25 & 3.61 \\
\hline \multirow[t]{3}{*}{ Linear Stage } & Duty cycle & 0.61 & 0.74 & 0.67 & 0.68 & 0.08 \\
\hline & Frequency & 8.12 & 9.77 & 8.5 & 8.95 & 1.14 \\
\hline & Scaling Gain & 0.14 & 0.19 & 0.16 & 0.17 & 0.38 \\
\hline
\end{tabular}

Due to multiple reasons, it is not feasible to report exact parameter values that generate the most realistic haptic sensations on normal bone. First, there is no available measure which describe a normal bone and there is often a range of bone that falls within this category. Also, different surgeons have different perceptions when it comes to haptics. Different cues are combined for each individual to form a perception. It can also be quite difficult trying to remember what something feels like. However, according to the statistical results, we can express that if we perform more observations, we are $95 \%$ confident that the tuned values are within the confidence interval. Further studies is required to see whether $95 \% \mathrm{CI}$ is an appropriate range to be used in the training. 


\subsubsection{Discussion of Research Question No. 2}

\section{How are the rotary stage parameters influenced in the presence of linear effects?}

As discussed earlier, rotary stage parameters that were tuned in the previous study are once again tuned in this work since the presence of the linear stage haptic effects might have affected the participants' haptic perception in tuning the rotary stage parameters. Moreover, a few modifications were applied to the implementation of the rotary stage haptic model.

Leung's study reported the median value for the tuned parameters as it is illustrated in Table 7.2. Also, the $95 \%$ confidence interval and median of the rotary stage parameters obtained in the current study are shown in the same table.

Table 7.2: Comparison of current study's rotary stage parameters with previous study

\begin{tabular}{|c|c|c|c|c|}
\hline \multirow{2}{*}{ Parameter } & \multicolumn{2}{|c|}{ Current Study(Outliers removed) } & Previous Study \\
\cline { 2 - 5 } & $95 \%$ CI (lower Boundary) & $95 \%$ CI (upper boundary) & Median & Median \\
\hline \hline Detent interval & 1.36 & 2.27 & 1.87 & 0.6 \\
Detent width & 0.36 & 0.58 & 0.47 & 0.69 \\
Detent magnitude & 0.45 & 0.74 & 0.57 & 0.53 \\
Viscous friction coeff. & 10.66 & 15.83 & 14.75 & 3.5 \\
\hline
\end{tabular}

The two studies reveal very close median values for the detent magnitude. The previous study's median value falls in the range of confidence interval of the current study. Median values for the detent width are close. However, the previous study's median value appear 
to be larger than CI upper boundary in the current study. Significant increase occurs for the viscous friction coefficient, rising from 3.5 to 14.75, placing considerably far from the current study's CI. The larger the viscous friction coefficient is, the more resistive torque the user feels when rotating the probe. The increase in this parameter could be attributed to the effect of adding the translational degree of freedom. When the user applies vertical forces to get motion along the axis of the pedicle, the haptic effects produced in the rotary stage may have felt less notable and the surgeon prefers a higher value to still perceive the resistive force, compared to the situation where he did not have to push (in Leung's study $[47])$.

Another noticeable change is seen in the detent interval, increasing from 0.6 to 1.87 . The previous study's median value is much smaller than the lower bound of the current study's CI. As discussed in the Section 4.3.1, vibration effects were not properly simulated in the previous work and did not provide much variation in terms of frequency. As a result of correcting the implementation, the surgeons generally tuned the detents to be felt further apart.

\subsubsection{Discussion of Research Question No. 3}

\section{Do the results differ across different expertise levels?}

We would like to determine whether significant differences exist among resident, fellow and expert surgeons. We can state the null hypothesis as following

$H_{0}$ : Mean is equal across different expertise levels

The one way analysis of variance (ANOVA) is a statistical technique that test if any of the means are different from each other within a dataset [54]. An assumption for ANOVA test is that multiple data samples have equal variances. Levene's test is used to analyze 
the variance across the three group of surgeons with different expertise [54]. The Levene's test returns a $p$-value in the range $[0,1]$ that shows how statistically significant is the difference in the variance. Outliers are excluded from the data before performing Levene's test. Therefore, the sample size for senior surgeons, fellow surgeons and resident surgeons becomes 2,3 and 5 for rotary stage parameters and 2, 4, 4 for linear stage parameters, respectively. The resulting $p$-value for each parameter is shown in Table 7.3.

Table 7.3: Levene's statistical test result

\begin{tabular}{|c|c|c|c|c|c|c|c|}
\hline & $\begin{array}{l}\text { Detent } \\
\text { Interval }\end{array}$ & $\begin{array}{l}\text { Detent } \\
\text { Width }\end{array}$ & $\begin{array}{l}\text { Detent } \\
\text { Magnitude }\end{array}$ & $\begin{array}{l}\text { Viscous } \\
\text { Friction Coeff. }\end{array}$ & Duty Cycle & Frequency & Scaling Gain \\
\hline P-value & 0.69 & 0.027 & 0.088 & 0.122 & 0.35 & 0.03 & 0.14 \\
\hline
\end{tabular}

In Levene's test, if the $p$-value is less than some significance level, typically 0.05 , then there is difference in the variance. Small values for the detent width in the rotary stage and the frequency in the linear stage indicate that they do not have homogeneity of variance and cannot use ANOVA as a result. For all other parameters, however, the variance is equal among groups as their corresponding $p$-values are larger than the significance level.

Table 7.4 illustrates mean and standard deviation of all parameters for each group of surgeons. 
Table 7.4: Summary statistic based on expertise level

\begin{tabular}{|c|c|c|c|c|}
\hline Parameter name & Expertise level & $\mathrm{N}$ & Mean & Std. Deviation \\
\hline \multirow[t]{3}{*}{ Detent interval } & Senior & 2 & 1.85 & 0.56 \\
\hline & Fellow & 3 & 1.89 & 0.81 \\
\hline & Resident & 5 & 1.76 & 0.69 \\
\hline \multirow[t]{3}{*}{ Detent width } & Senior & 2 & 0.40 & 0.28 \\
\hline & Fellow & 3 & 0.40 & 0.11 \\
\hline & Resident & 5 & 0.55 & 0.11 \\
\hline \multirow[t]{3}{*}{ Detent magnitude } & Senior & 2 & 0.85 & 0.14 \\
\hline & Fellow & 3 & 0.61 & 0.24 \\
\hline & Resident & 5 & 0.48 & 0.08 \\
\hline \multirow[t]{3}{*}{ Viscous friction coeff. } & Senior & 2 & 15 & 0.00 \\
\hline & Fellow & 3 & 14.16 & 2.75 \\
\hline & Resident & 5 & 12.00 & 4.63 \\
\hline \multirow[t]{3}{*}{ Duty cycle } & Senior & 2 & 0.70 & 0.00 \\
\hline & Fellow & 4 & 0.69 & 0.07 \\
\hline & Resident & 4 & 0.65 & 0.12 \\
\hline \multirow[t]{3}{*}{ Frequency } & Senior & 2 & 8.25 & 0.35 \\
\hline & Fellow & 4 & 10.06 & 0.9 \\
\hline & Resident & 4 & 8.18 & 0.47 \\
\hline \multirow[t]{3}{*}{ Scaling gain } & Senior & 2 & 0.15 & 0.06 \\
\hline & Fellow & 4 & 0.18 & 0.04 \\
\hline & Resident & 4 & 0.16 & 0.01 \\
\hline
\end{tabular}


ANOVA is performed for the five eligible parameters and results are shown in Table 7.5 .

Table 7.5: ANOVA test result

\begin{tabular}{|c|c|c|c|c|c|}
\hline & Detent Interval & Detent Magnitude & Viscous Friction Coeff. & Duty Cycle & Scaling Gain \\
\hline \hline P-value & 0.96 & 0.07 & 0.59 & 0.73 & 0.75 \\
\hline
\end{tabular}

All $p$-values are larger than the significance level (0.05). Hence, for none of these parameters the null hypothesis cannot be rejected. However, detent magnitude has a very small $p$-value. With more participants we may find enough evidence to reject the null hypothesis.

To examine the null hypothesis for the two other parameters (detent width and frequency) Welch's test (see Table 7.6) is conducted. Welch's test is to test for the equality of means given that the variances are unequal and samples are independent [54].

Table 7.6: Welch test result

\begin{tabular}{|c|c|c|}
\hline & Detent Width & Frequency \\
\hline \hline P-value & 0.44 & 0.07 \\
\hline
\end{tabular}

Welch test result reveals that the null hypothesis cannot be rejected for any of the two parameters. This conclusion, however, does not necessarily mean that the null hypothesis is accepted. Noting that $p$-value for the frequency is just slightly over the significance level, the null hypothesis may be rejected with more data. 
In order to closely look at the variation of the tuned values among surgeons with different level of expertise, error bars are used. In Figure 7.2, upper and lower boundaries of the error bar indicate the variance and the middle circle indicates the mean of data for each parameter.
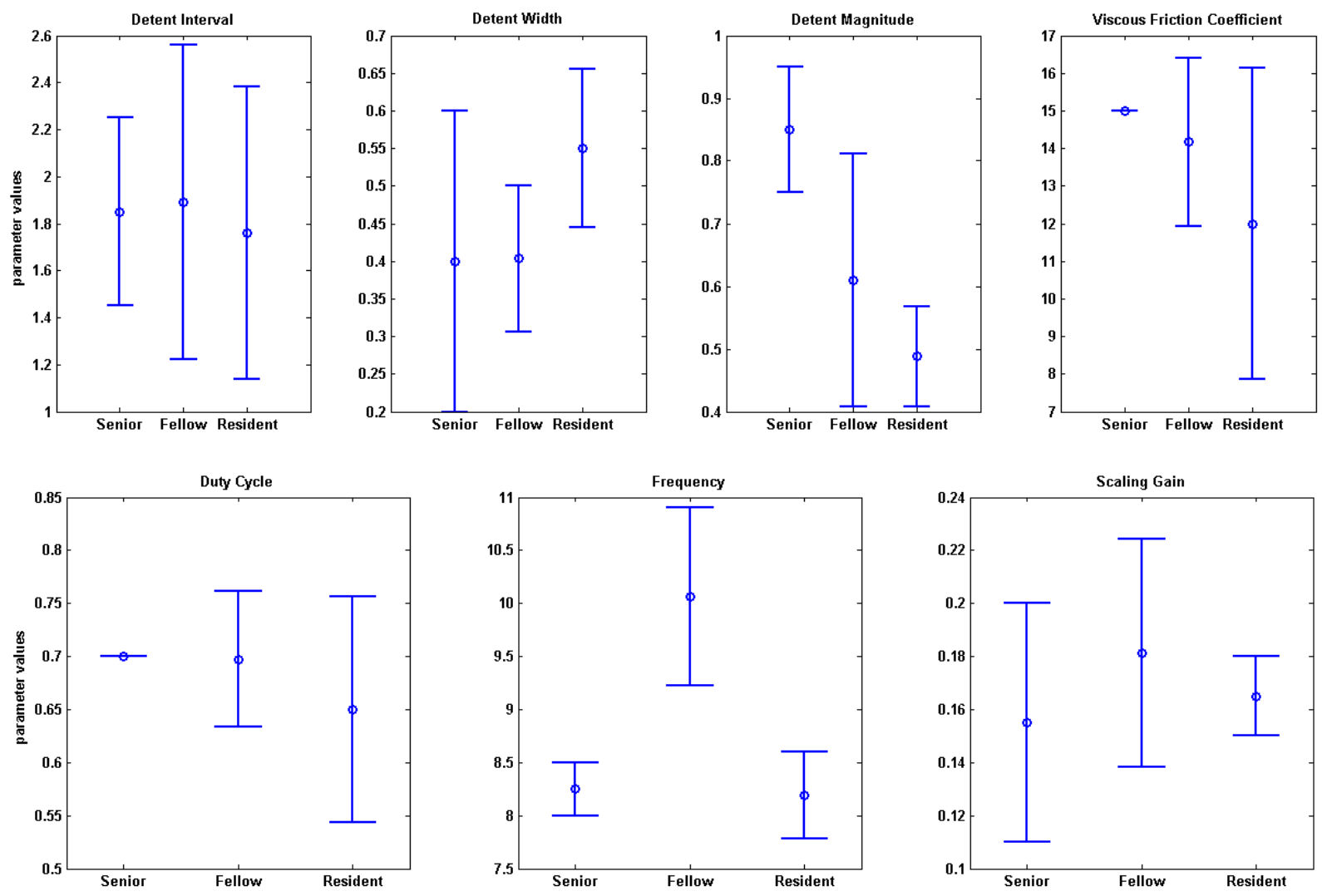

Figure 7.2: Comparison of variance among surgeons with different expertise level with respect to mean, outliers are removed

Among 7 parameters, senior surgeons have narrower range for five parameters including the detent interval, the detent magnitude, the viscous friction coefficient, the duty cycle, and 
the frequency of vibration. Interestingly, they have agreement for two of the parameters, the viscous friction coefficient in rotary stage and the duty cycle in linear stage. However, more senior surgeons should be recruited to further validate such results. Knowing that

there are not too many experienced surgeons in this field, we were limited in our ability to recruit more people within the time frame of this research.

Range of variance was not significantly different between fellow and resident surgeons. For some parameters, the error bar was shorter for fellows than residents and for some, resident surgeons have smaller variance than fellows. This could be attributed to the fact that even fellow surgeon do not perform this kind of surgery on a regular basis. This kind of surgery relies very much on haptic memory and requires constant practice to maintain and enhance precise haptic skills.

\subsubsection{Discussion of Research Question No. 4}

Could the simulator serve as a useful tool in the education of surgical trainees for teaching channel creation in pedicle screw insertion?

Eleven surgeon participants were asked to give feedback on the developed surgery simulator using the questionnaire detailed in the Section 6.6. Results are shown using the bar chart in Figure 7.3. 


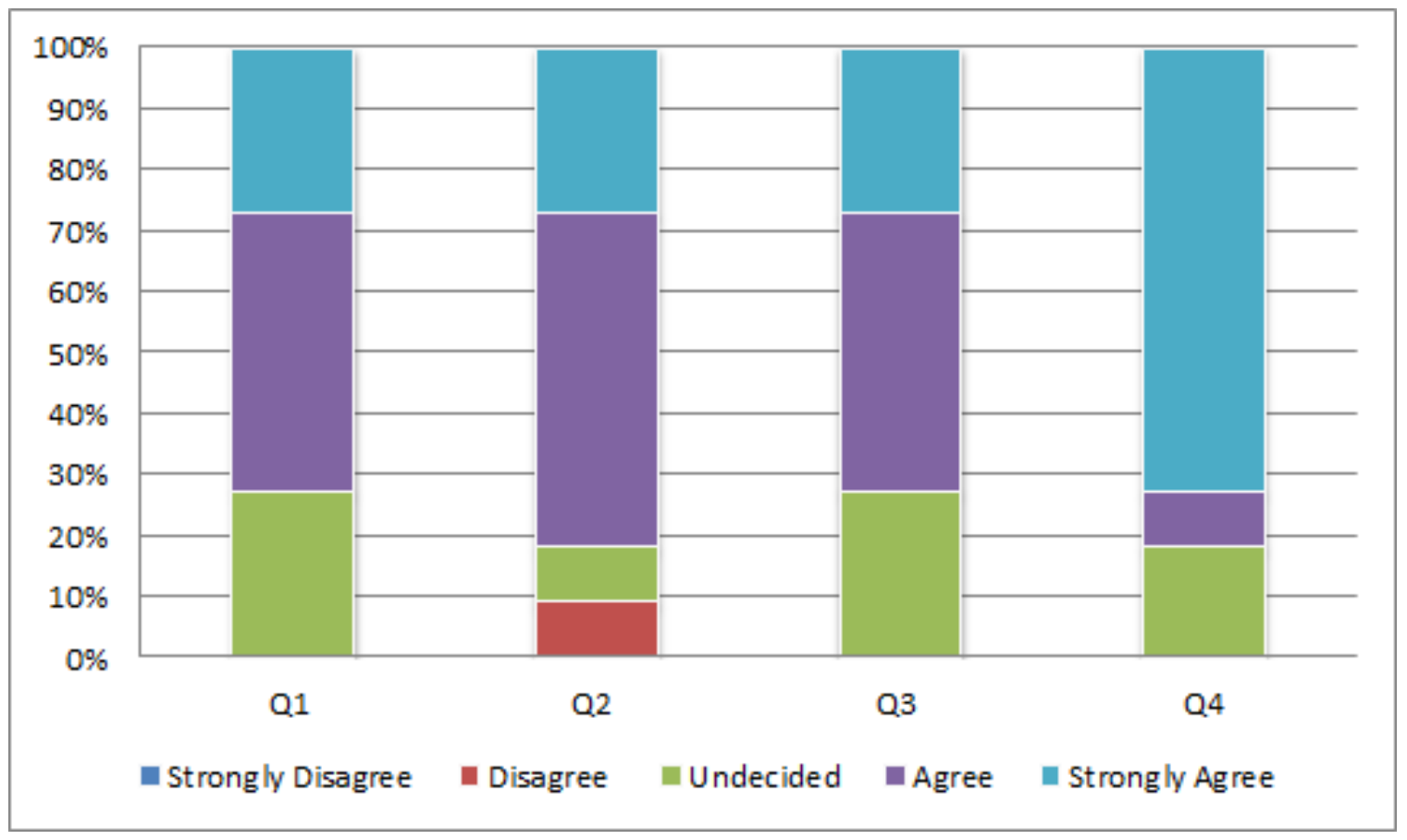

Figure 7.3: Summary of questionnaire results for 11 surgeon participants. Q1 : The haptic sensations associated with the rotation of the probe was simulated realistically. Q2: The haptic sensations associated with the linear progression of the probe was simulated realistically. Q3: Overall, the simulator produced realistic haptic sensations felt during probe channeling. Q4: The simulator could potentially be a useful tool for teaching pedicle screw insertion surgery.

The chart illustrates the percentages of participants on five response categories (strongly disagree, disagree, undecided, agree, strongly agree) in four questions. For all questions, fellow and expert surgeons either strongly agreed or agreed. Undecided response were mainly from the resident surgeons with the least experience. Surgeons were often positive toward the study, believing the simulation was a close representation of using a probe on 
an actual vertebrae.

According to [55], satisfaction ratings can be treated as continuous data and be compared to a specific benchmark or goal in order to be able to measure satisfaction. Assuming that the benchmark for the questionnaire is positive (non-neutral) with the rate of 3.5/5, we would like to determine whether the user's satisfaction exceeds this benchmark. 90\% confidence interval is computed for each question and is shown in Table 7.7. CI is calculated using t-distribution for the sample size of 11 and two-tailed 10 percent critical value.

Table 7.7: Comparing satisfaction rating of simulator realism and teaching tool potential to an agreement benchmark $(3.5 / 5.0)$

\begin{tabular}{|c|c|c|c|c|}
\hline Question & Benchmark & Mean & Std. Dev. & 90\% Confidence Interval \\
\hline \hline Q1 & 3.5 & 4 & 0.77 & $3.58-4.42$ \\
Q2 & 3.5 & 4 & 0.89 & $3.51-4.49$ \\
Q3 & 3.5 & 4 & 0.77 & $3.58-4.42$ \\
Q4 & 3.5 & 4.55 & 0.82 & $4.10-4.99$ \\
\hline
\end{tabular}

As can be seen, for each of the four questions, confidence interval exceeds the benchmark Of $3.5 / 5$. We can be $90 \%$ confident that participant's satisfaction score is more positively than neutral. For the last question (simulator as teaching tool), we can be more than $90 \%$ confident that participants would agree $(4 / 5)$ that the simulator can potentially be a useful tool for teaching pedicle screw insertion. 


\subsection{Summary}

Pilot studies prior to official testings are explained in this chapter. Then, data collected in the clinical testing is statistically analyzed and the findings are presented. A benchmark reference set of the values for haptic model parameters are calculated. A comparison is made on the rotary stage parameters between the current study and the previous work. A test is performed to investigate the difference across surgeons of different expertise. Positive feedback was provided by participants on the usefulness of the simulator. 


\section{Chapter 8}

\section{Conclusion and Future Works}

This work has extended the previous work in which the rotary stage of a haptic training simulator for scoliosis surgery was designed and built. Here, by adding a linear degree of freedom and coupling the two stages, the haptic robot can simulate the haptic effects associated with the two degree of freedom involved in the pedicle screw insertion: rotation and linear progression. This chapter will present the main findings in this study.

\subsection{General Conclusion}

There are relatively few surgeons in Canada performing pedicle screw insertion. Therefore, participant recruitment was a major challenge for the clinical tuning of parameters given the limited time of the study. Moreover, not all surgeons do the surgery regularly and most residents develop their surgical skill set on cadavers' bone rather than the healthy bone on younger patients who comprise the highest volume of scoliosis surgical cases. Despite the small sample size, among the participants is a senior surgeon who performs a major 
proportion of all surgeries in Canada of this type.

The participants' expertise were quite varied by multiple factors including their level of training, number of performed operations in OR, number of performed operations on bony tissue, and the number of performed operations specifically for the pedicle screw insertion. Some had more surgical experience with robotic tools. The neurosurgeon less often perform this procedure; sometimes only once or twice a year.

According to the questionnaire survey, all of the senior and fellow surgeon participants found the haptic training simulator to be a useful tool in teaching probe channeling in pedicle screw insertion. The current device is capable of simulating the various force and torque effects a surgeon feels in this surgery. The current simulator is a first of its kind in the field of spine surgery, with the ability of replicating the haptic sensations in free-hand probe channeling through the bone with high-fidelity haptic feedback.

Study results suggest that senior surgeons have a relatively smaller range of tuned parameter variation in comparison to the resident and fellow surgeons for most haptic model parameters, indicating that senior surgeons have developed a more precise and consistent haptic perception. Noting that there are only two senior surgeons in the study, certainly additional experienced surgeons are required for further validation. However, these initial results indicate the possibility that these tighter tuned parameter variations could be used to screen trainees for how close their skill level approaches those of the expert surgeons.

Some haptic parameters are more sensitive than others in an operation. According to the ANOVA and Welch test results, for two parameters (the detent magnitude in the rotary stage and the frequency in the linear stage) significance value is very close to the significance level (0.05). This indicates that by more data we may find evidence to reject 
the null hypothesis that surgeons with different expertise levels are same. If the difference is found across surgeons, one practical way to see if training helped is to observe the pattern in these two parameters. They can be employed as a metric to differentiate the trainees in terms of haptic skill performance.

In summary, in this work, we have developed the first haptic simulator for the emulation of pedicle screw placement with free-hand technique. This simulator is the first of its kind that is able to simulate high forces created in the free-hand technique. We have also created a methodology for tuning model parameters.

\subsection{Future Work}

The work in this thesis can be continued by broadening the study sample size. Balancing the sample size of orthopedic surgeons and neurosurgeons, as well as surgeons with different expertise level would make the results better reflect the population and would improve the finding's statistical power.

The focus of the current study was on simulating haptics sensation for the normal healthy bone. Pedicle screw insertion is performed on a large variety of patients who present with various spinal disorders. Future work involves data collection for other anatomical scenarios such as osteoporotic bone employing the same testing protocol explained in Chapter 6.

As discussed, breaching through the pedicle wall is one of the important adverse event that surgeons should be aware of during the surgery. It is important that surgeons become familiar with this sensation in a safe and repeatable environment. Unfortunately, our attempt to imitate this scenario was not successful in this study due to the hardware 
limitation. Replacing the linear motor with a faster one and revising the overall design to incorporate lighter hardware components are among technical suggestions that may make breach simulation feasible. Also, closed loop control techniques would be considered with the improved hardware.

In order to increase realism and create a more effective simulation, a human interaction interface is necessary. The apparatus can be covered by a physical interface that provides the look of an actual patient's body. Preparing a visual and haptic replication of the other steps in pedicle screw insertion would enhance the realism of the overall simulation.

Since this haptic simulator is planned to ultimately serve as a surgical training tool, future direction for this work includes determining appropriate training techniques and investigating ways for surgical skill assessment. The setup would employ the reference set of values tuned within the clinical testing for various anatomical scenarios. Through an interactive training system, the proficiency of the surgeons can be tracked.

Another interesting study would be investigating the force and torque profiles of the surgeons during the simulated environment and compare it to those on an actual patient/cadaver. As a part of the testing protocol in this study, the force and torque profile of each participant was recorded. The research group at the University of Toronto designed and integrated an instrumented surgical probe that can measure probe force and torque signals. Figure 8.1 shows a sample of the force and torque profile that were measured in one of the testing trials when the participant imitates probe channeling on the tuned haptic simulator. 

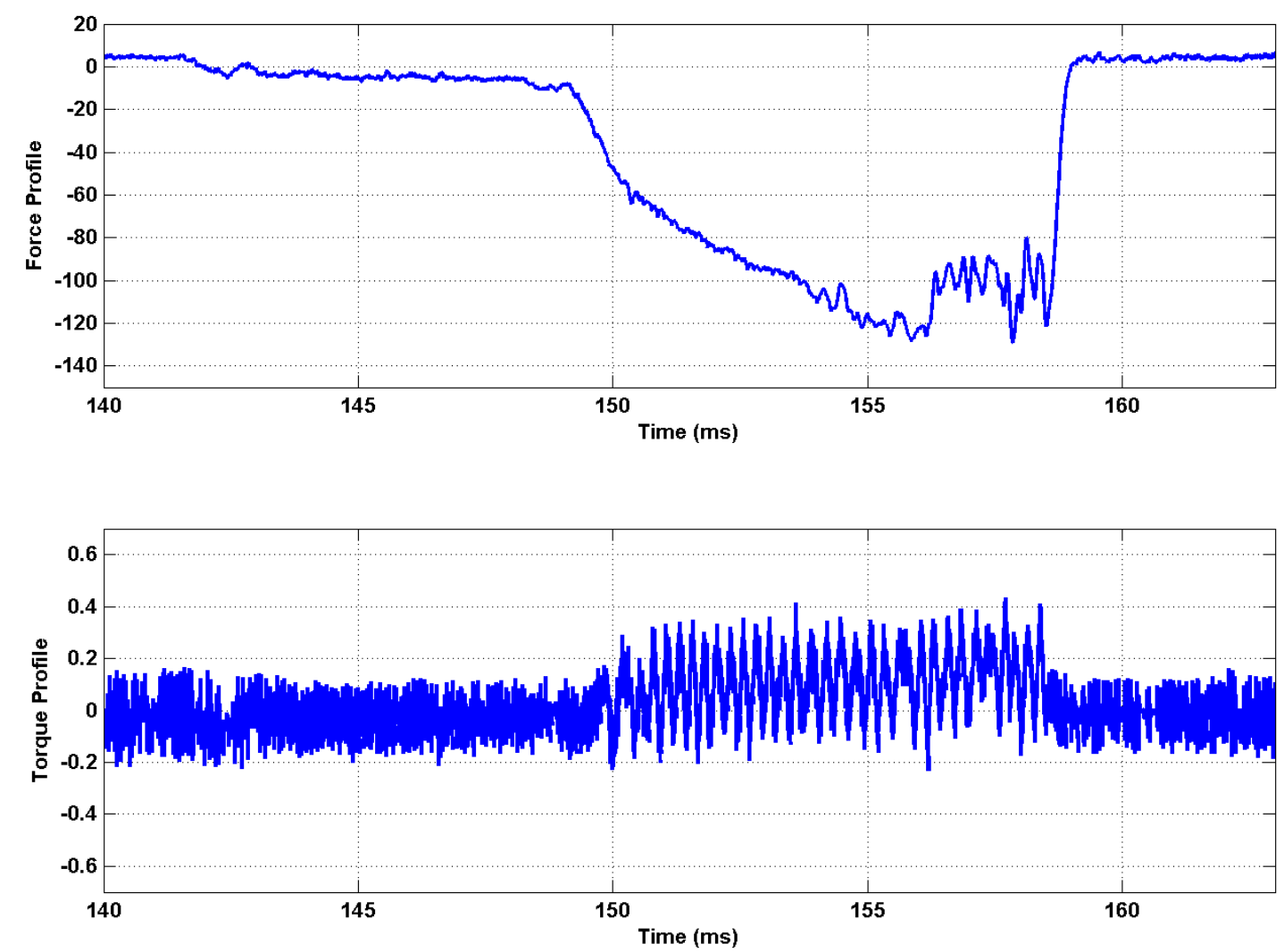

Figure 8.1: Force and torque profile of a participant while using the haptic simulator

This work generally simulates the interaction between a surgical probe and bone. Importantly, it is a simulation that focuses on manual surgical gestures, versus surgical gestures that use robotic tools such as drills. It also capable of emulating high forces in such applications. Therefore, it can be employed, with further developments, to any applications within the medical field that focuses on manual tool interaction with bone. Further development may involve adding other degrees of freedom and/or visual components. 


\subsection{Summary}

This simulator, being the first of its kind in the simulation of pedicle screw insertion, received positive feedback on its usefulness in surgical training. Further testing is required to validate the statistical results since the participants' clinical experience were varied. Further technical developments would be an asset to simulate other surgical scenarios. 
APPENDICES 


\section{Appendix A}

\section{System Components}

\section{Computer}

The computer's operating system is Microsoft Windows 7 running on a core i7 processor (3.4 GHz Clock) and it has 8GB installed RAM.

\section{Data Acquisition}

For rotary stage, a Sensoray Model 626 PCI data acquisition card is employed along with a MultiQ-PCI terminal board to read from encoder and sending motor commands to the amplifier with the maximum sampling rate of $1 \mathrm{KHz}$. The same DAQ is used for acquiring position sensor data through the analog input port of the terminal board. An analog output port of this board is also used for sending the control signal to the linear motor's amplifier. The force sensor data is, however, collected through a separate DAQ card provided by ATI.

On the PCI terminal, analog input for the position sensor is placed in parallel with a resistor-capacitor circuit. This RC circuit facilitates filtering the input signal with a cut-off frequency of $200 \mathrm{~Hz}$. 


\section{Amplifiers}

Two AMC 50AI Brush Type PWM Servo Amplifier are responsible for driving the voltage to the rotary and linear actuators. The amplifiers are powered by a 57V DC power supply. The linear motor's amplifier is set to work in voltage mode with the gain of 1.2 to maximize the output voltage range. The rotary motor's amplifier, on the other hand is configured in the current mode with the gain of 1 .

\section{Rotary motor}

A Kollmorgen JR16M4CH ServoDisc motor is used for the rotary stage. It can produce up to $36.8 \mathrm{Nm}$ of torque.

\section{Linear motor}

A 9" Stroke Deluxe, 100 lb Force Fast Actuator is used. It has a load capacity of 100 lbs. It has fixed limit switches and operates quietly.

\section{Encoder}

A SICK DFS60 (model DFS60A-S4AC65536) encoder is used for measuring angular position. It has the resolution of 65536 pulses/rev.

\section{Position Sensor}

A Celesco SP2 string pot is employed for measuring position.

\section{Force/Torque Sensor}

An ATI IP68 Nano25 is used for this purpose. This sensor connects to the commputer through ATI DAQ F/T.

\section{MATLAB}

Simulations were performed in real-time in MATLAB 2014a (32 bit). Through the Real-Time Windows Target library, analog input and output blocks are accessible. 
Using analog input blocks, the sensors' data are collected from the DAQ and using analog output block the control signals can be transmitted to the DAQ and from there to the motor drivers.

\section{A.1 Graphical User Interface}

The command interface that was used for tuning the model parameters is MATLAB Graphical User Interface (GUI). As seen in Figure A.2 and A.1, it contains sliders and text boxes for accessing the features of the simulation model that is developed in Simulink. 


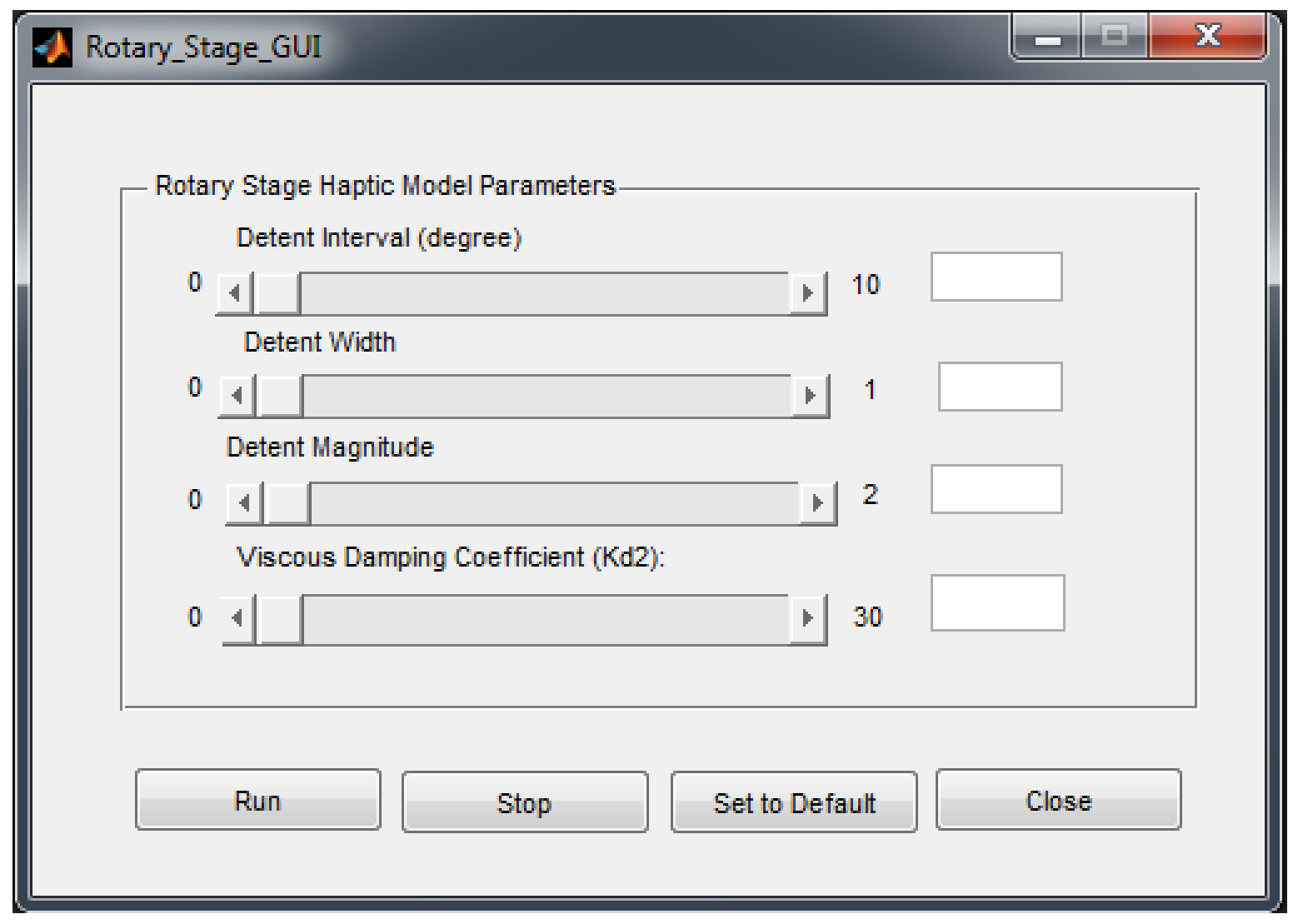

Figure A.1: Graphical user interface used for tuning rotary stage parameters 


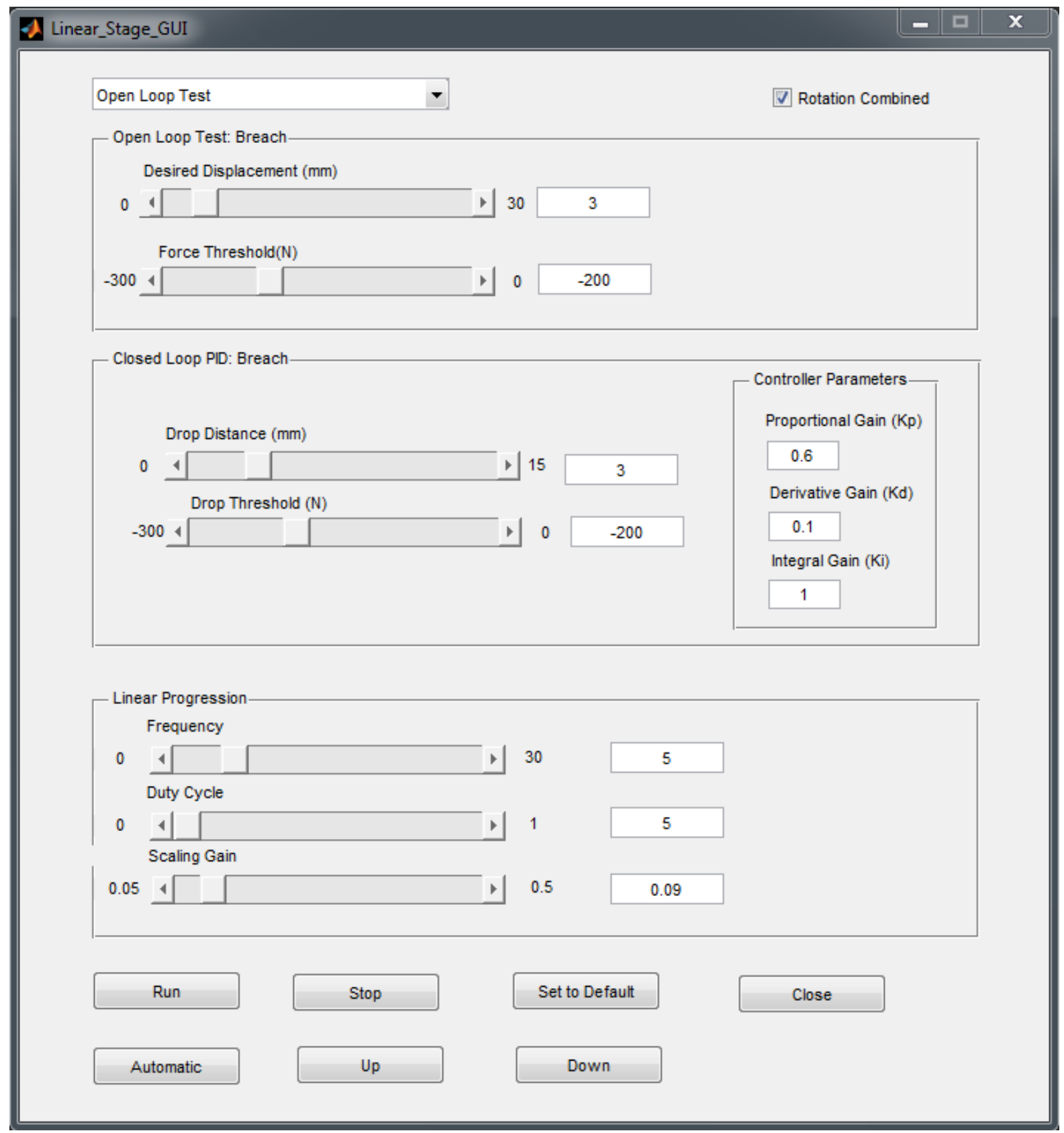

Figure A.2: Graphical user interface used for tuning linear stage parameters 
Appendix B

Ethics Material 


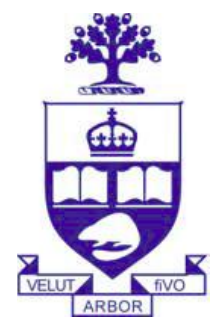

Information Sheet

\begin{abstract}
Study title: BIOMECHANICAL DETERMINANTS OF SURGICAL OUTCOMES IN ADOLESCENTS WITH A PAEDIATRIC SPINAL DEFORMITY. A pilot study that examines the potential application of haptics for the purpose of computer simulation of spine surgery.
\end{abstract}

The investigative team includes the following:

Professor Karl F Zabjek, PhD Dr.

Reinhard Zeller, MD, FRCSC

Professor Anne Agur, PhD

Professor Heather Carnahan, $\mathrm{PhD}$

Professor Elaine Biddis, $\mathrm{PhD}$

Professor Sunita Mathur, PhD

Dr. Stephen Lewis, MD, FRCSC

Professor David Wang, PhD

Ms. Regina Leung, BSc.

Mr. Kevin Walker, MSc.

Ms. Maryam Moafimadani, BSc

Funding Source: Canadian Institute of Health Research (CIHR)

\title{
1. Introduction
}

Presently, the orthopaedic community has limited access to the necessary imaging and biomechanical tools that will permit the simulation of intra-operative surgical gestures. For proceedures that involve surgical instrumentation of the spine, pedicle screw insertion is a fundamental skill. This skill is traditionally trained using the 'see one do one' approach. However due to the complexity of this skill there is a significant need to identify optimal simulation models that will assist in the training of Orthopaedic Trainees.

The overall objective of this proposal is to understand how the different models of surgical simulation and the level of surgeon expertise will influence the performance the mechanics of a surgical gesture.

Objective 1: Compare the biomechanics of a surgical gesture (pedicle screw insertion) that is performed using three different surgical simulation models.. These three simulation models 
include: A) Cadaver (HSim); B) Simple Simulation Model (SimpSim); C) Comprehensive Simulation Model (CompSim).

Objective 2: Compare the biomechanics of a surgical gesture that is performed by three groups of Orthopaedic specialists with different levels of expertise. These levels of expertise include:

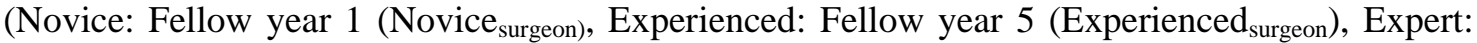
Practicing Orthopaedic Surgeon (Practicing surgeon$_{\text {). }}$.

\section{Study Procedures}

For this study you will be requested to visit one testing session that is located in the Musculoskeletal Anatomy Laboratory at the University of Toronto. This visit will last approximately $1.5-2$ hours.

\section{Testing sessions}

During the testing sessions, the investigator will do the following:

1. Put on sensors on the arms, legs, trunk, pelvis that will measure arm motions, muscle activity.

2. Perform the simulated act of pedicle screw insertion on three different models (HSim; SimpleSim; CompSim).

3. Repeat the simulated act of pedicle screw insertion using these three different models.

4. Provide structured and unstructured feedback at how 'similar'/'dissimilar' these models are.

\section{Discomforts and Risks}

There are minimal risks associated with this study. The risk and discomfort experienced during the biomechanical assessment does not exceed that of the expereinced during the performance of an intra-operative proceedure. The placement of sensors on the skin is non-invasive, with adhesion between the sensor and the skin obtained by the use of hypoalergenic double sided tape. When the tape is removed from the participant, there is a small degree of discomfort that is equivalent to the removal of a band aid. Participation in this study may affect your self perception of yourlevel of skill associated with pedicle screw insertion. If you identify this as a concern you will be referred to the University of Toronto Fellowships program councilling program.

\section{Benefits}

There are no direct benefits related to this study. Through visiting the Musculoskeletal Anatom Laboratory at the University of Toronto the participants will have an opportunity to learn more about state of the art methods used to study movement and simulate surgical skills training.

\section{Data Storage and Confidentiality}

All information collected during this study will be stored for 7 years in a safe, secure and locked location at University of Toronto. At the end of 7 years, all information collected during this study will be destroyed (paper will be shredded and electronic documents will be deleted). We will take care to protect your confidentiality. Your name and identifying personal information will not be used on any of the data collection forms. In addition to the researchers involved in this study, individuals from regulatory authorities or the Research Ethics Board who are involved in 
monitoring and auditing of studies may be granted access to the information collected during this study.

\section{Voluntary Participation}

Your participation in this study is strictly voluntary. You are able to withdraw at any time before, during, or after the study. You may withdraw by contacting any of the study investigators and indicating that you are no longer interested in participating. If you should decline to participate, it will not affect any future relationship with the investigators or facility.

\section{Expenses}

You will not have to pay for any of the procedures involved in this study. You will be reimbursed for reasonable travel expenses.

\section{Copy of Information}

You will be provided with written information about this study and a copy of this information and consent form. If you are interested, we will arrange for you to receive a copy of the results of this study once it has been completed.

\section{Questions and Concerns}

If you have any questions or concerns, please contact the Principal Investigator of the study, Dr.

Karl Zabjek at 416-978-5072. This study has been approved by Health Sciences - Research Ethical Board (REB) at the University of Toronto.

If you have any concerns regarding the ethics of the study, please contact the Director of the Research Ethics Board, Rachel Zand at 416-946-3389. 


\section{$\underline{\text { Participant Consent Form }}$}

\section{BIOMECHANICAL DETERMINANTS OF SURGICAL OUTCOMES IN}

ADOLESCENTS WITH A PAEDIATRIC SPINAL DEFORMITY. A pilot study that examines the potential application of haptics for the purpose of computer simulation of spine surgery.

Name (please print clearly):

I have read the Letter of Information and have had any questions answered to my satisfaction.

I understand that I will be participating in "BIOMECHANICAL DETERMINANTS OF SURGICAL OUTCOMES IN ADOLESCENTS WITH A PAEDIATRIC SPINAL

DEFORMITY. A pilot study that examines the potential application of haptics for the purpose of computer simulation of spine surgery." and I will be asked to perform simulated surgical gestures.

I understand that my participation in this study is voluntary and that I may withdraw at any time without negative consequences. I understand that every effort will be made to maintain the confidentiality of the data now and in the future.

I have read the above statements and freely consent to participate $\mathrm{n}$ this research:

Signature: Date:

Witness Name: Date:

Signature:

Should you have any questions, concerns or complaints, feel free to contact the investigators (SpineHealth@gmail.com); the faculty advisor, Karl Zabjek (416-978-5072, k.zabjek@utoronto.ca); or the Ethics Review Office (416-946-3273, ethics.review@utoronto.ca). 


\section{References}

[1] Paul B Suh and G Dean Macewen. Idiopathic scoliosis in males a natural history study. Spine, 13(10):1091-1094, 1988.

[2] Alok Sud and Athanasios I Tsirikos. Current concepts and controversies on adolescent idiopathic scoliosis: Part i. Indian journal of orthopaedics, 47(2):117, 2013.

[3] Stuart L Weinstein, Lori A Dolan, Jack CY Cheng, Aina Danielsson, and Jose A Morcuende. Adolescent idiopathic scoliosis. The Lancet, 371(9623):1527-1537, 2008.

[4] Han Jo Kim, John S Blanco, and Roger F Widmann. Update on the management of idiopathic scoliosis. Current opinion in pediatrics, 21(1):55-64, 2009.

[5] Keith Bridwell. Spinal Anatomy: Vertebral Column. http://www. spineuniverse. com/anatomy/vertebral-column.

[6] Online MSK Radiology Book. Retrieved from University of Washington, Department of Radiology. http://www.rad.washington.edu/academics/academic-sections/ msk/teaching-materials/online-musculoskeletal-radiology-book/ scoliosis. 
[7] Alok Sud and Athanasios I Tsirikos. Current concepts and controversies on adolescent idiopathic scoliosis: Part ii. Indian journal of orthopaedics, 47(3):219, 2013.

[8] AR Vaccaro, SJ Rizzolo, TJ Allardyce, M Ramsey, J Salvo, RA Balderston, and JM Cotler. Placement of pedicle screws in the thoracic spine. $J$ Bone Joint Surg [Am], 77:1200-1206, 1995 .

[9] Al-Hassan Aly, Howard J Ginsberg, and Richard SC Cobbold. On ultrasound imaging for guided screw insertion in spinal fusion surgery. Ultrasound in medicine $\mathcal{E}^{3}$ biology, 37(4):651-664, 2011.

[10] Tobias A Mattei, Murilo S Meneses, Jerônimo B Milano, and Ricardo Ramina. Freehand technique for thoracolumbar pedicle screw instrumentation: critical appraisal of current state-of-art. Neurology India, 57(6), 2009.

[11] Seung-Jae Hyun, Yongjung J Kim, Gene Cheh, Seung Hwan Yoon, and Seung-Chul Rhim. Free hand pedicle screw placement in the thoracic spine without any radiographic guidance: technical note, a cadaveric study. Journal of Korean Neurosurgical Society, 51(1):66-70, 2012.

[12] Yongjung J Kim, Lawrence G Lenke, et al. Thoracic pedicle screw placement: freehand technique. Neurology India, 53(4):512, 2005.

[13] Frank Phillips and Safdar N Khan. Treatment of Complex Cervical Spine Disorders, An Issue of Orthopedic Clinics, volume 43. Elsevier Health Sciences, 2012.

[14] Han Jo Kim, Marinus de Kleuver, and Keith Luk. Inserion of pedicle screws. https://www2. aofoundation.org/wps/portal/! ut/p/c0/04_ SB8K8xLLM9MSSzPy8xBz9CP0os3hng7BARydDRwN39yBTAyMvLwOLUA93I4MQE_ 
2CbEdFAF3RnT4! /?soloState=true\&contentUrl=/srg/popup/additional_ material/55/X160-InsertionOfPedicleScrews . jsp\#JumpLabelNr1.

[15] Yongjung J Kim, Lawrence G Lenke, Keith H Bridwell, Yongsun S Cho, and K Daniel Riew. Free hand pedicle screw placement in the thoracic spine: is it safe? Spine, 29(3):333-342, 2004.

[16] Bernd Wegener, Christof Birkenmaier, Andreas Fottner, Volkmar Jansson, and Hans Roland Dürr. Delayed perforation of the aorta by a thoracic pedicle screw. European Spine Journal, 17(2):351-354, 2008.

[17] John M Hicks, Amit Singla, Francis H Shen, and Vincent Arlet. Complications of pedicle screw fixation in scoliosis surgery: a systematic review. Spine, 35(11):E465E470, 2010.

[18] Vincent Y Wang, Cynthia T Chin, Daniel C Lu, Justin S Smith, and Dean Chou. Free-hand thoracic pedicle screws placed by neurosurgery residents: a CT analysis. European Spine Journal, 19(5):821-827, 2010.

[19] Amer F Samdani, Ashish Ranade, Daniel M Sciubba, Patrick J Cahill, M Darryl Antonacci, David H Clements, and Randal R Betz. Accuracy of free-hand placement of thoracic pedicle screws in adolescent idiopathic scoliosis: how much of a difference does surgeon experience make? European Spine Journal, 19(1):91-95, 2010.

[20] Robert Riener and Matthias Harders. VR for medical training. In Virtual Reality in Medicine, pages 181-210. Springer, 2012.

[21] Timothy R Coles, Dwight Meglan, and N John. The role of haptics in medical training simulators: a survey of the state of the art. Haptics, IEEE Transactions on, 4(1):5166, 2011. 
[22] Randy S Haluck, Renee L Marshall, Thomas M Krummel, and Michael G Melkonian. Are surgery training programs ready for virtual reality? a survey of program directors in general surgery. Journal of the American College of Surgeons, 193(6):660-665, 2001.

[23] Corinna E Lathan, Michael R Tracey, Marc M Sebrechts, Deborah M Clawson, and Gerald A Higgins. Using virtual environments as training simulators: Measuring transfer. Handbook of virtual environments: Design, implementation, and applications, pages 403-414, 2002.

[24] JE Korteling, EAPB Oprins, and VL Kallen. Measurement of effectiveness for training simulations. In RTO-MP-SAS-095 Cost-Benefit Analysis of Military Training. Paper presented at the SAS Workshop held in Amsterdam, The Netherlands, pages 5-6, 2012.

[25] Frank Tendick, Michael Downes, Tolga Goktekin, Murat Cenk Cavusoglu, David Feygin, Xunlei Wu, Roy Eyal, Mary Hegarty, and Lawrence W Way. A virtual environment testbed for training laparoscopic surgical skills. Presence: Teleoperators and Virtual Environments, 9(3):236-255, 2000.

[26] Kenneth Salisbury, Francois Conti, and Federico Barbagli. Haptic rendering: introductory concepts. Computer Graphics and Applications, IEEE, 24(2):24-32, 2004.

[27] Abdulmotaleb El Saddik. Haptics technologies: bringing touch to multimedia. Springer, 2011.

[28] Mohamed Benali-Khoudja, Moustapha Hafez, Jean-Marc Alexandre, and Abderrahmane Kheddar. Tactile interfaces: a state-of-the-art survey. In Int. Symposium on Robotics, volume 31, 2004.

[29] Grigore C Burdea. Keynote address: haptics feedback for virtual reality. In Proceedings of international workshop on virtual prototyping. Laval, France, pages 87-96, 1999. 
[30] Dan Morris. Haptics and physical simulation for virtual bone surgery. PhD thesis, Stanford University, 2006.

[31] David Prytherch and Mairghread McLundie. So what is haptics anyway? Research Issues in Art, Design and Media, 2:29, 2002.

[32] Christopher R Wagner, Nicholas Stylopoulos, and Robert D Howe. The role of force feedback in surgery: Analysis of blunt dissection. In Symposium on Haptic Interfaces for Virtual Environment and Teleoperator Systems, pages 73-79. Citeseer, 2002.

[33] Yan Zhang, Roger Phillips, James Ward, and Sandhya Pisharody. A survey of simulators for palpation training. Medicine Meets Virtual Reality 17-Nextmed: Design for the Well Being, 444, 2009.

[34] Jeffrey Berkley, George Turkiyyah, Daniel Berg, Mark Ganter, and Suzanne Weghorst. Real-time finite element modeling for surgery simulation: An application to virtual suturing. Visualization and Computer Graphics, IEEE Transactions on, 10(3):314$325,2004$.

[35] Arnold D Steinberg, Philip G Bashook, James Drummond, Seema Ashrafi, and Milos Zefran. Assessment of faculty perception of content validity of periosim (c), a haptic-3d virtual reality dental training simulator. Journal of dental education, 71(12):1574$1582,2007$.

[36] T Sohmura, H Hojo, M Nakajima, K Wakabayashi, M Nagao, S Iida, T Kitagawa, M Kogo, T Kojima, K Matsumura, et al. Prototype of simulation of orthognathic surgery using a virtual reality haptic device. International journal of oral and maxillofacial surgery, 33(8):740-750, 2004. 
[37] Chee-Kong Chui, Jackson SK Ong, Zheng-Yi Lian, Zhenlan Wang, Jeremy Teo, Jing Zhang, Chye-Hwang Yan, Sim-Heng Ong, Shih-Chang Wang, Hee-Kit Wong, et al. Haptics in computer-mediated simulation: training in vertebroplasty surgery. Simulation \& Gaming, 37(4):438-451, 2006.

[38] Jackson Shin-Kiat Ong, Chee-Kong Chui, ZL Wang, Jing Zhang, Jeremy Choon-Meng Teo, Chye Hwang Yan, Sim Heng Ong, Chee Leong Teo, and Swee-Hin Teoh. Biomechanical modeling of bone-needle interaction for haptic rendering in needle insertion simulation. In Control, Automation, Robotics and Vision, 2006. ICARCV'06. 9th International Conference on, pages 1-6. IEEE, 2006.

[39] JB Ra, SM Kwon, JK Kim, J Yi, KH Kim, HyunWook Park, K-U Kyung, D-S Kwon, HS Kang, ST Kwon, et al. Spine needle biopsy simulator using visual and force feedback. Computer Aided Surgery, 7(6):353-363, 2002.

[40] Dong-Soo Kwon, Ki-Uk Kyung, Sung Min Kwon, Jong Beom Ra, Hyun Wook Park, Heung Sik Kang, Jianchao Zeng, and Kevin R Cleary. Realistic force reflection in a spine biopsy simulator. In Robotics and Automation, 2001. Proceedings 2001 ICRA. IEEE International Conference on, volume 2, pages 1358-1363. IEEE, 2001.

[41] Amir Manbachi, Richard SC Cobbold, and Howard J Ginsberg. Guided pedicle screw insertion: techniques and training. The Spine Journal, 14(1):165-179, 2014.

[42] Shawn Klein, Cari M Whyne, Raphael Rush, and Howard J Ginsberg. CT-based patient-specific simulation software for pedicle screw insertion. Journal of spinal disorders $\&$ techniques, 22(7):502-506, 2009. 
[43] Behzad Eftekhar, Mohammad Ghodsi, Ebrahim Ketabchi, and Saman Rasaee. Surgical simulation software for insertion of pedicle screws. Neurosurgery, 50(1):222-224, 2002.

[44] Rohit Rambani, James Ward, and Warren Viant. Desktop-based computer-assisted orthopedic training system for spinal surgery. Journal of surgical education, 71(6):805809, 2014.

[45] Ryan Schmidt. Spinaltap: An architecture for real-time vertebrae drilling simulation. Technical report, Department of Computer Sciences at the University of Calgary, 2002.

[46] Cristian J Luciano, P Pat Banerjee, Brad Bellotte, G Michael Lemole Jr, Michael Oh, Fady T Charbel, and Ben Roitberg. Learning retention of thoracic pedicle screw placement using a high-resolution augmented reality simulator with haptic feedback. Neurosurgery, 69(Suppl OPERATIVE):ons14, 2011.

[47] Regina Leung. Design of a haptic simulator for pedicle screw insertion in pediatric scoliosis surgery. Master's thesis, University of Toronto, 2013.

[48] Stefan H Thomke. Experimentation matters: unlocking the potential of new technologies for innovation. Harvard Business Press, 2003.

[49] Neville Hogan. Impedance control: An approach to manipulation: Part iiimplementation. Journal of dynamic systems, measurement, and control, 107(1):8-16, 1985.

[50] N Hogan and SP Buerger. Impedance and interaction control, robotics and automation handbook, 2005.

[51] DJ Ewins. Modal testing: Theory, practice, and application, 2000. Research Studies Press LTD., Baldock, Hertfordshire, England, 171:415-437. 
[52] Roxy Peck, Chris Olsen, and Jay Devore. Introduction to statistics and data analysis. Cengage Learning, 2008.

[53] Charles Henry Brase and Corrinne Pellillo Brase. Understandable statistics: concepts and methods. Cengage Learning, 2011.

[54] Andy Field. Discovering statistics using IBM SPSS statistics. Sage, 2013.

[55] Jeff Sauro and James R Lewis. Quantifying the user experience: Practical statistics for user research. Elsevier, 2012.

[56] Vincent Hayward, Oliver R Astley, Manuel Cruz-Hernandez, Danny Grant, and Gabriel Robles-De-La-Torre. Haptic interfaces and devices. Sensor Review, 24(1):16$29,2004$.

[57] Susan J Lederman and Roberta L Klatzky. Haptic perception: A tutorial. Attention, Perception, \&3 Psychophysics, 71(7):1439-1459, 2009.

[58] JuEun Lee, B Arda Gozen, and O Burak Ozdoganlar. Modeling and experimentation of bone drilling forces. Journal of biomechanics, 45(6):1076-1083, 2012.

[59] C Canudas De Wit, P Noel, A Aubin, and B Brogliato. Adaptive friction compensation in robot manipulators: Low velocities. The International journal of robotics research, 10(3):189-199, 1991.

[60] National Institute of Arthritis, Musculoskeletal, and Skin Diseases (NIAMS). Scoliosis In Children And Adolescents. http://www.niams.nih.gov/Health_Info/ Scoliosis/\#normal.

[61] Brani Vidakovic. Statistics for Bioengineering Sciences: With MATLAB and WinBUGS Support. Springer, 2011. 
[62] MATLAB. version 8.3.0 (R2014a). The MathWorks Inc., Natick, Massachusetts, 2014. 